DFTT $46 / 09$

EPHOU 09-003

July, 2009

\title{
Formulation of Supersymmetry on a Lattice as a Representation of a Deformed Superalgebra
}

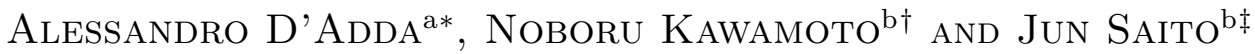 \\ a INFN sezione di Torino, \\ and \\ Dipartimento di Fisica Teorica, Universita di Torino \\ I-10125 Torino, Italy \\ b Department of Physics, Hokkaido University \\ Sapporo, 060-0810 Japan
}

\begin{abstract}
The lattice superalgebra of the link approach is shown to satisfy a Hopf algebraic supersymmetry where the difference operator is introduced as a momentum operator. The breakdown of the Leibniz rule for the lattice difference operator is accommodated as a coproduct operation of (quasi)triangular Hopf algebra and the associated field theory is consistently defined as a braided quantum field theory. Algebraic formulation of path integral is perturbatively defined and Ward-Takahashi identity can be derived on the lattice. The claimed inconsistency of the link approach leading to the ordering ambiguity for a product of fields is solved by introducing an almost trivial braiding structure corresponding to the triangular structure of the Hopf algebraic superalgebra. This could be seen as a generalization of spin and statistics relation on the lattice. From the consistency of this braiding structure of fields a grading nature for the momentum operator is required.
\end{abstract}

\footnotetext{
*E-mail: dadda@to.infn.it

${ }^{\dagger}$ E-mail: kawamoto@particle.sci.hokudai.ac.jp

${ }^{\ddagger}$ E-mail: saito@particle.sci.hokudai.ac.jp
} 


\section{Introduction}

We consider that a constructive definition of regularized supersymmetric field theory is getting increasingly important. There are several reasons: Phenomenologically there is an expectation that superparticles might be discovered by LHC experiment in the near future. If the supersymmetry becomes reality, obviously we need to formulate supersymmetric field theory constructively. The formulation should provide a basis for the numerical study of nonperturbative supersymmetry phenomenology. It is natural to expect that a lattice formulation of supersymmetry may play a crucial rôle just like the lattice QCD is playing an important rôle as the only numerical mean for the strong interaction phenomenology. We expect that a fermionic counterpart of QCD region may exist in the new energy scale.

Secondly, it is not obvious that the lattice fermion problems [1, 2] are well understood from the lattice regularization point of view. It is, however, a general consensus that the chiral fermion problem is solved for lattice QCD [3, 4, 5]. One may say that species doublers of chiral fermion on a lattice are lattice artifacts so that it would have been better if they were not there. It was, however, claimed that these extra species doubler degrees of freedom is exactly the needed one and corresponds to the extended twisted supersymmetry degrees of freedom [6, 7, 8, 9]. It was shown that the twisted supersymmetry can be derived by the Dirac-Kähler twisting procedure [10] in any dimensions: $\mathcal{N}=2$ in two dimensions, $\mathcal{N}=4$ in three dimensions, and $\mathcal{N}=4$ in four dimensions which coincides with the twisting derived by Marcus [11. In these formulations the fermionic internal degrees of freedom can be defined semilocally on a lattice to be compatible with differential form nature of Dirac-Kähler fermion [6, 7, 8, 9, 12, 13. This type of the correspondence has been anticipated by the old works [14. It turns out that the lattice Dirac-Kähler fermion formulation [15] was, however, proved to be equivalent to the staggered fermion formulation [16] with an introduction of mild noncommutativity between differential forms and fields to accommodate the modified Leibniz rule for lattice difference operator. These results suggest that the regularization of fermions on a lattice naturally leads to a necessity of supersymmetry in a fundamental way.

In the path integral formulation of field theory, fermionic fields are treated as Grassmann odd variables and thus have an anti-commuting nature in compatible with spin and statistics theorem which requires Lorentz invariance exactly [17]. Since the Lorentz invariance is broken on the lattice it is not obvious that this anti-commuting nature of fermions at the lattice constant level is the mandatory requirement. In this paper we explore a possibility that commuting and anti-commuting nature of fields are modified with an introduction of mild noncommutativity in compatible with the lattice Leibniz rule of difference operator. This may be identified as a generalization of spin and statistics on a lattice.

Trials for the formulation of supersymmetry on a lattice have a long history. Since lattice does not have infinitesimal translational invariance, there are various difficulties to formulate supersymmetry algebra which includes an infinitesimal translation generator. To overcome the difficulties, various approaches and formulations have been proposed so far. If we focus on the treatment of the algebraic aspects of lattice supersymmetry, there are essentially three possible approaches:

1. keeps the continuum superalgebra approximately with lattice corrections;

2. keeps exactly only a subalgebra of the continuum superalgebra which doesn't contain the momentum operators;

3. deforms the continuum superalgebra into a lattice version of superalgebra, and keeps exactly the full sector of this lattice superalgebra.

There is a long list of many trials of the first approach summarized in [18, 19, 20] and the previous 
references are therein, where superalgebra is kept up to the lattice corrections. There are some later developments [21, 22, 23, 24]. In this approach one has to see how the superalgebra is restored in the continuum limit. In order to see the recovery of supersymmetry for the whole range of coupling constant, it is inevitable to find reliable methods for numerical analyses. Influenced by the developments of renormalization group analyses and Ginsparg-Wilson relation of chiral symmetry analyses for lattice QCD, there have been recent systematic applications of the methods to supersymmetric models [25, 26, 27].

In the second approach [28, 29, 30, 31, 32, 33, 34, 35, 36], only one or two nilpotent supercharges are preserved exactly. It is again particularly important to examine how the full continuum supersymmetry is recovered in the continuum limit. In this approach the importance of accidental symmetry is stressed so that the recovery of supersymmetry in the infrared region is expected due to the suppression of relevant operators by the partial exactness of the total supersymmetry algebra [37, 36]. In fact in some specific cases it can be shown that only the part of supersymmetry which is realized on the lattice is enough to suppress non-manageable fine-tuning in the continuum limit [28, 30, 31, 32, 36, 38]. One can then use such models as constructive definition of corresponding continuum supersymmetric models. We should, however, note that in extracting the sector of superalgebra which can be preserved on the lattice, extended supersymmetry and its twisting procedure [39, 10] play an important rôle .

In the third approach [6, 7, 8, 12, 13, 34, 40], one defines lattice version of superalgebra where the momentum operators in the continuum superalgebra are replaced by finite difference operators on the lattice. This seems the most natural and naïve deformation of continuum algebra. Nevertheless, it is not straightforward due to the following obvious reason. Since a finite difference operator, not being an infinitesimal operator, is not rigorously an element of algebra, that deformed "superalgebra" is not strictly an algebra in the usual sense. This is actually not simply a terminological issue, but is crucial in the formulation. Namely, finite difference operator does not obey the Leibniz rule, which is nothing but equivalent to say that the operator is not an element of algebra. On the other hand, because of the nilpotency of Grassmann parameters, "normal" supercharge would always obey exactly the Leibniz rule. This mismatch of natures of finite difference operators and supercharges makes the naïve realization of lattice deformed superalgebra in the above sense difficult. What we are going to follow in this paper is in fact the formulation struggling to give an answer to this difficult situation, which was originally proposed in [6, 7, 8]. We call this formulation as link approach as a whole or DKKN formalism when we have more stress on the algebraic aspect of the formulation. In this approach, we introduce the notion of modified Leibniz rule to overcome this difficulty.

Despite of the invention of the modified Leibniz rule, the link approach still faces its incompleteness. Firstly, it is rather unclear whether this additionally introduced modified Leibniz rule is totally consistent and acceptable to express the "symmetry" of a quantum field theory, because it is in any case different from the standard Lie algebraic symmetry. As an answer to this issue we will present that this modification is indeed consistently introduced, utilizing the fact that the deformed algebra forms a Hopf algebra which generalizes the Lie algebraic symmetry, and that a quantum field theory which has a Hopf algebraic symmetry can be constructed at least perturbatively thanks to the previously formulated framework known as braided quantum field theory (BQFT) [41]. For this argument, it is important to identify the DKKN superalgebra as a rigorous Hopf algebra which obeys a set of axioms to prescribe the Hopf algebra. It is also crucial to correctly determine the braiding structure on the representation space of the Hopf algebra. With the use of the BQFT formulation, we can derive a series of Ward-Takahashi identities corresponding to the Hopf algebraic symmetry, which would give a clear physical interpretation of the deformed symmetry.

The second and most crucial aspect to be clarified in the link approach is on the "inconsistency" raised in 42, which claims that the modified Leibniz rule inevitably leads to a problem 
due to the ordering ambiguity caused essentially by the asymmetric nature of the deformation. In order to clarify the ordering problem, a matrix formulation for one dimensional model was explicitly analysed. It was shown that there is no ambiguity at the superfield level but the problem remains at the component level [40]. Fortunately, our Hopf algebraic description also resolves this problem: given the Hopf algebraic symmetry together with the appropriate treatment of the braiding structure of BQFT, we will show that this difficulty no longer exists. The braiding structure, which could be interpreted as a kind of generalized statistics or a "mild noncommutativity", is again the key ingredient for this argument. The treatment for the gauge theory is outside of the scope of this paper.

In the recent investigations it is stressed that the second approach formulated by orbifold construction and the third approach of the link construction are equivalent [34, 35]. It was already noticed that a particular choice of a constant vector parameter makes the scalar supercharge shiftless and $\mathcal{N}=D=2$ super Yang-Mills action of the link construction coincides with that of orbifold construction [7]. It was, however, stressed that supercharges carrying shifts are not supersymmetry invariant [34, 35]. Therefore in the symmetric choice of the parameters in the link construction, all the supercharges carry shifts and thus no supersymmetry exists, although the corresponding action has larger discrete chiral and spacetime symmetries than that of orbifold construction [35. This criticism is due to the non-standard definition of the shifted (anti-)commutators. In this paper we intend to stress exactly on this point that all supercharges of the link construction preserves deformed lattice supersymmetry exactly, where shifting nature plays a crucial rôle .

Recently it was shown as a no-go theorem that a proper definition of product of lattice fields naturally leads to a breakdown of Leibniz rule of lattice differential operator under the conditions of translational invariance and locality on the lattice [43]. It was also shown that an extension of blocked symmetry transformation realizing Ginsparg-Wilson relation to supersymmetric case leads to only consistent solution of SLAC-type derivative [44] which is in fact consistent with the above no-go theorem [27. It is known, however, that SLAC-derivative is a highly non-local differential operator. It should be noted that the modified nature of the lattice Leibniz rule or equivalently the deformation of supersymmetry algebra is compatible with the results of those analyses.

This paper is organized as follows. In Section 2 we will make a brief review of the fundamental structures of the link formulation in somewhat generalized form and its above mentioned difficulties. In Section 3 we will give a description how to treat the superalgebra on the lattice as a deformed/modified algebra in the scheme of the Hopf algebra theory. We will list all the necessary and sufficient formulae which form the whole structures of a Hopf algebra. We also derive the explicit form of the braiding which is necessary for the total consistency of the representation. Twisting of our Hopf algebra will be discussed, too, which naturally explains our lattice theory should have the braiding or equivalent noncommutativity. We will illustrate how a quantum field theory with this Hopf algebraic symmetry can be perturbatively defined entirely based on the general formulation of BQFT. As a concrete example we show a two dimensional $\mathcal{N}=2$ Wess-Zumino model. We then give the conclusion of the paper and discuss some remaining issues in the last section. In the appendix we give a brief summary of Hopf algebra to fix the notation and terminology appeared in the text. 


\section{General Framework of the Link Formulation of the Dirac- Kähler Twisted Supersymmetry on a Lattice}

\subsection{Generality of the Formulation}

The principle of the link approach [6, 7, 8] to a realization of a supersymmetric theory on a lattice is based on a simple assumption that the superalgebra in the continuum 1

$$
\begin{gathered}
\left\{Q_{A}, Q_{B}\right\}=2 \tau_{A B}^{\mu} P_{\mu}, \\
{\left[Q_{A}, P_{\mu}\right]=\left[P_{\mu}, P_{\nu}\right]=0}
\end{gathered}
$$

has some natural counterpart on the lattice

$$
\begin{gathered}
\left\{Q_{A}^{\text {lat }}, Q_{B}^{\text {lat }}\right\}=2 \tau_{A B}^{\mu} P_{\mu}^{\text {lat }}, \\
{\left[Q_{A}^{\text {lat }}, P_{\mu}^{\text {lat }}\right]=\left[P_{\mu}^{\text {lat }}, P_{\nu}^{\text {lat }}\right]=0 .}
\end{gathered}
$$

Here $\tau_{A B}^{\mu}$ is just a constant coefficient, and $Q_{A}^{\text {lat }}$ and $P_{\mu}^{\text {lat }}$ are understood both as deformed operators on the lattice which come back to $Q_{A}$ and $P_{\mu}$, respectively, in the naïve continuum limit;

$$
\lim _{a \rightarrow 0} Q_{A}^{\mathrm{lat}}=Q_{A}, \quad \lim _{a \rightarrow 0} P_{\mu}^{\mathrm{lat}}=P_{\mu} .
$$

We require that

$$
\sum_{x} P_{\mu}^{\text {lat }} \varphi(x)=0
$$

for the "momentum" operator $P_{\mu}^{\text {lat }}$ and any field $\varphi(x)$ on the lattice. This is because, in the continuum, the general superinvariance of Lagrangian is up to total divergence which vanishes under the integral, and the same structure should be true for the "exact" supersymmetry on the lattice, for which the property above is necessary. We would also require the translational invariance and (semi-)locality for the operator $P_{\mu}^{\text {lat }}$ so that the whole theory would have these properties. Another possible requirement might be the Hermiticity (or the reflection (Osterwalder-Schrader) positivity [45] of transfer matrices on the lattice [47]), but we don't force it here because it is related to the subtlety of the doubling phenomenon [1, 2] for which we defer the discussion to later sections.

The simplest candidates for the "momentum" operator $P_{\mu}^{\text {lat }}$ would be the finite difference operators on the lattice,

$$
P_{\mu}^{\mathrm{lat}}=i \partial_{ \pm \mu}, \quad i \partial_{\mu}^{\mathrm{s}}, \quad \text { etc. }
$$

where

$$
\begin{aligned}
\partial_{+\mu} \varphi(x) & :=\frac{1}{a}(\varphi(x+a \hat{\mu})-\varphi(x)) & & \text { (forward difference operator), } \\
\partial_{-\mu} \varphi(x) & :=\frac{1}{a}(\varphi(x)-\varphi(x-a \hat{\mu})) & & \text { (backward difference operator), } \\
\partial_{\mu}^{\mathrm{s}} \varphi(x) & :=\frac{1}{2}\left(\partial_{+\mu}+\partial_{-\mu}\right) \varphi(x) & & \text { (symmetric difference operator) } \\
& =\frac{1}{2 a}(\varphi(x+a \hat{\mu})-\varphi(x-a \hat{\mu})) & & \text { (s) }
\end{aligned}
$$

where $\hat{\mu}$ is the unit vector to the direction of $x^{\mu}$ and $a$ is the lattice constant 2 . The symmetric difference is self anti-Hermitian: $\left(\partial_{\mu}^{\mathrm{s}}\right)^{\dagger}=-\partial_{\mu}^{\mathrm{s}}$, while the others are anti-Hermitian conjugate to

\footnotetext{
${ }^{1}$ The notation here is schematic; indices $A$ and $B$ could contain both spinor and internal d.o.f., and their conjugate as well.

${ }^{2}$ We always keep the lattice constant $a$ explicitly in this paper unless otherwise specified.
} 
each other; $\left(\partial_{ \pm \mu}\right)^{\dagger}=-\partial_{\mp \mu}$. An immediate consequence of using these finite difference operators is that they break the Leibniz rule, or put it milder, obey the modified Leibniz rule as in

$$
\begin{aligned}
\partial_{ \pm \mu}\left(\varphi_{1} \varphi_{2}\right)(x) & =\partial_{ \pm \mu} \varphi_{1}(x) \varphi_{2}(x)+\varphi_{1}(x \pm a \hat{\mu}) \partial_{ \pm \mu} \varphi_{2}(x) \\
& =\partial_{ \pm \mu} \varphi_{1}(x) \varphi_{2}(x \pm a \hat{\mu})+\varphi_{1}(x) \partial_{ \pm \mu} \varphi_{2}(x) \\
& =\partial_{ \pm \mu} \varphi_{1}(x) \varphi_{2}(x)+\varphi_{1}(x) \partial_{ \pm \mu} \varphi_{2}(x) \pm a \partial_{ \pm \mu} \varphi_{1}(x) \partial_{ \pm \mu} \varphi_{2}(x)
\end{aligned}
$$

and

$$
\begin{aligned}
\partial_{\mu}^{\mathrm{s}}\left(\varphi_{1} \varphi_{2}\right)(x) & =\partial_{\mu}^{\mathrm{s}} \varphi_{1}(x) \varphi_{2}(x-a \hat{\mu})+\varphi_{1}(x+a \hat{\mu}) \partial_{\mu}^{\mathrm{s}} \varphi_{2}(x) \\
& =\partial_{\mu}^{\mathrm{s}} \varphi_{1}(x) \varphi_{2}(x+a \hat{\mu})+\varphi_{1}(x-a \hat{\mu}) \partial_{\mu}^{\mathrm{s}} \varphi_{2}(x) \\
& =\partial_{\mu}^{\mathrm{s}} \varphi_{1}(x) \varphi_{2}(x)+\varphi_{1}(x) \partial_{\mu}^{\mathrm{s}} \varphi_{2}(x)+\frac{a}{2}\left(\partial_{+\mu} \varphi_{1}(x) \partial_{+\mu} \varphi_{2}(x)-\partial_{-\mu} \varphi_{1}(x) \partial_{-\mu} \varphi_{2}(x)\right)
\end{aligned}
$$

Although superficially the breaking term in each case of the Leibniz rule is proportional to the lattice constant $a$, it is not in general of higher order in the continuum limit, due to the contributions from the cut off scale region of the momentum $\partial_{ \pm \mu} \varphi(x) \sim \mathcal{O}(1 / a)$ [24]. Note also that the last term of (2.10) is proportional to a total difference $\partial_{-\mu}\left(\partial_{+\mu} \varphi_{1}(x) \partial_{+\mu} \varphi_{2}(x)\right)$, so that one may consider that this breaking of the Leibniz rule is irrelevant under a summation over the whole lattice sites. But this is true only for the product of two fields, so that might be a good property only in a free theory, not in an interacting case. (Even in the free case there is an associated doubler problem for the anti-Hermitian symmetric difference. We will see this later in more detail.) One might also try to impose a constraint on the fields to make the breaking terms vanish, but this would only result in a nonlocal formulation [18. Thus, as long as we use the simple difference operators (2.5), we can't naïvely neglect the breaking of the Leibniz rule. In fact, it is more generally shown [27, 43] that we have to admit the breaking of the Leibniz rule of any "momentum" operators on a lattice, unless we allow nonlocal operators like so-called SLAC derivative [44] or, say, many multiflavors. These facts are already enough for the lattice counterpart of the superalgebra (2.2) to lose the nature of strict Lie superalgebra, which is the most evident and crucial obstacle to formulate supersymmetry entirely based on the superalgebra on a lattice.

One possibility to overcome the situation is to interpret the superalgebra on the lattice (2.2) as a "deformed" Lie superalgebra with the deformation parameter that vanishes in the continuum limit. This is in fact the basic strategy in the link approach as we can see in what follows.

Since the r.h.s. of (2.2) obeys the modified Leibniz rule, it is natural to deform the algebra so that the generators in the l.h.s. also obeys a modified Leibniz rule. In the link approach, the central ansatz is that the supercharge $Q_{A}^{\text {lat }}$ obeys the Leibniz rule of the form 3

$$
Q_{A}^{\mathrm{lat}}\left(\varphi_{1} \varphi_{2}\right)(x)=Q_{A}^{\mathrm{lat}} \varphi_{1}(x) \varphi_{2}(x)+(-1)^{\left|\varphi_{1}\right|} \varphi_{1}\left(x+a_{A}\right) Q_{A}^{\mathrm{lat}} \varphi_{2}(x),
$$

where $x+a_{A}$ is to be interpreted as denoting an extended lattice site which goes to $x$ in the naïve continuum limit. Introducing a translation or shift operator $T_{a_{A}}$ in a "fundamental" representation such that

$$
T_{a_{A}} \varphi(x)=\varphi\left(x+a_{A}\right),
$$

the Leibniz rule can be written as

$$
\begin{aligned}
Q_{A}^{\mathrm{lat}}\left(\varphi_{1} \varphi_{2}\right)(x) & =Q_{A}^{\mathrm{lat}} \varphi_{1}(x) \varphi_{2}(x)+(-1)^{\left|\varphi_{1}\right|} T_{a_{A}}\left(\varphi_{1} T_{a_{A}}^{-1} Q_{A}^{\mathrm{lat}} \varphi_{2}\right)(x), \\
\text { i.e. } \quad T_{a_{A}}^{-1} Q_{A}^{\mathrm{lat}}\left(\varphi_{1} \varphi_{2}\right)(x) & =\left(T_{a_{A}}^{-1} Q_{A}^{\mathrm{lat}} \varphi_{1}\right)(x) \varphi_{2}\left(x-a_{A}\right)+(-1)^{\left|\varphi_{1}\right|} \varphi_{1}(x)\left(T_{a_{A}}^{-1} Q_{A}^{\mathrm{lat}} \varphi_{2}\right)(x),
\end{aligned}
$$

\footnotetext{
${ }^{3}$ Here $|\varphi|$ is 0 or 1 , depending on whether $\varphi$ is bosonic or fermionic, respectively.
} 
showing that the operator $T_{a_{A}}^{-1} Q_{A}^{\text {lat }}$ obeys a slightly different modified Leibniz rule. We may also write it in a symmetric form as

$T_{a_{A}}^{-1 / 2} Q_{A}^{\mathrm{lat}}\left(\varphi_{1} \varphi_{2}\right)(x)=\left(T_{a_{A}}^{-1 / 2} Q_{A}^{\mathrm{lat}} \varphi_{1}\right)(x) \varphi_{2}\left(x-a_{A} / 2\right)+(-1)^{\left|\varphi_{1}\right|} \varphi_{1}\left(x+a_{A} / 2\right)\left(T_{a_{A}}^{-1 / 2} Q_{A}^{\mathrm{lat}} \varphi_{2}\right)(x)$,

which is still a modified version of Leibniz rule. We could have begun with a little more generalized modification such as

$$
Q_{A}^{\mathrm{lat}}\left(\varphi_{1} \varphi_{2}\right)(x)=Q_{A}^{\mathrm{lat}} \varphi_{1}(x) \varphi_{2}\left(x+a_{A}^{\mathrm{r}}\right)+(-1)^{\left|\varphi_{1}\right|} \varphi_{1}\left(x+a_{A}^{\mathrm{l}}\right) Q_{A}^{\mathrm{lat}} \varphi_{2}(x),
$$

but with a redefinition of $Q_{A}^{\text {lat }}$ to $T_{a_{A}^{\mathrm{r}}}^{-1} Q_{A}^{\text {lat }}$ it is always equivalent to the original one (2.11), which can be seen in a similar fashion as in the above. Such a redefinition only causes a total difference in the algebra (2.2), hence the original form (2.11) suffices in general.

The field in the fundamental representation of the translation/shift operator (2.12) could be interpreted as a normal function on the lattice. If, by contrast, we introduce the "adjoint" representation of the translation/shift operator as in

$$
T_{a_{A}} \varphi(x) T_{a_{A}}^{-1}=\varphi\left(x+a_{A}\right),
$$

the Leibniz rule (2.11) can be written as

$$
\begin{aligned}
Q_{A}^{\mathrm{lat}}\left(\varphi_{1} \varphi_{2}\right)(x) & =Q_{A}^{\mathrm{lat}} \varphi_{1}(x) \varphi_{2}(x)+(-1)^{\left|\varphi_{1}\right|} T_{a_{A}} \varphi_{1}(x) T_{a_{A}}^{-1} Q_{A}^{\mathrm{lat}} \varphi_{2}(x), \\
\text { i.e. } \quad T_{a_{A}}^{-1} Q_{A}^{\mathrm{lat}}\left(\varphi_{1} \varphi_{2}\right)(x) & =T_{a_{A}}^{-1} Q_{A}^{\mathrm{lat}} \varphi_{1}(x) \varphi_{2}(x)+(-1)^{\left|\varphi_{1}\right|} \varphi_{1}(x) T_{a_{A}}^{-1} Q_{A}^{\mathrm{lat}} \varphi_{2}(x) .
\end{aligned}
$$

Now we can see that the operator $T_{a_{A}}^{-1} Q_{A}^{\text {lat }}$ obeys the usual exact Leibniz rule. We could write this further as

$$
T_{a_{A}}^{-1} Q_{A}^{\mathrm{lat}}\left(\varphi_{1} \varphi_{2}\right)(x) T_{a_{A}^{\prime}}=T_{a_{A}}^{-1} Q_{A}^{\mathrm{lat}} \varphi_{1}(x) T_{a_{A}^{\prime}} \varphi_{2}\left(x-a_{A}^{\prime}\right)+(-1)^{\left|\varphi_{1}\right|} \varphi_{1}(x) T_{a_{A}}^{-1} Q_{A}^{\mathrm{lat}} \varphi_{2}(x) T_{a_{A}^{\prime}},
$$

which shows the operator $T_{a_{A}}^{-1} Q_{A}^{\text {lat }} \overleftarrow{T}_{a_{A}^{\prime}}$, where the arrow denotes the multiplication from the right, follows a different Leibniz rule. We would thus again find that the original modified Leibniz rule (2.11) itself is equivalent to the more general form (2.15), and even to the usual Leibniz rule (2.17) with a suitable redefinition of the operator $Q_{A}^{\text {lat }}$. Notice that in this adjoint representation such a redefinition would change the algebra (2.2) in a nontrivial way, except for the case $a_{A}^{\mathrm{r}}=a_{A}^{\mathrm{l}}$ for which the algebra, or more precisely the "momentum" operator, would remain unchanged up to a total difference so that the "momentum" operator still obeys the modified Leibniz rule. In other words, unless $a_{A}^{\mathrm{r}}=a_{A}^{\mathrm{l}}$, we have a possibility to redefine both the operators $Q_{A}^{\text {lat }}$ and $P_{\mu}^{\text {lat }}$ so as to follow the usual Leibniz rule for which the usual representation would exist. This fact may play an important rôle for an explicit representation of the lattice superalgebra. Another point to observe is that, in the adjoint representation, field itself should be identified as an operator or a matrix which formally belongs, together with the shift operator, to an algebra (which would be a universal enveloping algebra of a Lie superalgebra, just as in a canonical quantization scheme.). The operator $T_{a_{A}}$, when multiplying from the left/right on a field, changes the property under a commutation of the field. For instance, suppose $\varphi_{1}(x)$ and $\varphi_{2}(x)$ commute with each other; $\varphi_{1}(x) \varphi_{2}(x)=\varphi_{2}(x) \varphi_{1}(x)$. Then $T_{a_{A}} \varphi_{1}(x)$ and $\varphi_{2}(x)$ no longer commute strictly, but commute with a shift in the sense that $T_{a_{A}} \varphi_{1}(x) \varphi_{2}(x)=T_{a_{A}} \varphi_{2}(x) T_{a_{A}}^{-1} T_{a_{A}} \varphi_{1}(x)=\varphi_{2}\left(x+a_{A}\right) T_{a_{A}} \varphi_{1}(x)$. This type of mild noncommutative nature doesn't simply occur in the fundamental case since $\left(T_{a_{A}} \varphi_{1}\right)(x) \varphi_{2}(x)=\varphi_{2}(x)\left(T_{a_{A}} \varphi_{1}\right)(x)$. We will see in the next chapter how the modified Leibniz rules in the fundamental representation (2.11), (2.13) and (2.14) can be more systematically treated in the framework of Hopf algebraic symmetry. Here in what follows we continue the general description mainly with the adjoint representation case. 
Suppose now $Q_{A}^{\text {lat }}$ also belongs to this same algebra that $T_{a_{A}}$ and $\varphi(x)$ form. The fact that the combination $T_{a_{A}}^{-1} Q_{A}^{\text {lat }}$ in (2.17) follows the usual Leibniz rule thus motivates us to write formally

$$
T_{a_{A}}^{-1} Q_{A}^{\mathrm{lat}}=: \hat{Q}_{A}^{\mathrm{lat}} \equiv i \operatorname{ad}\left(\hat{\mathcal{Q}}_{A}^{\mathrm{lat}}\right),
$$

which acts on a field ast

$$
\begin{gathered}
T_{a_{A}}^{-1} Q_{A}^{\mathrm{lat}} \varphi(x)=i \operatorname{ad}\left(\hat{\mathcal{Q}}_{A}^{\mathrm{lat}}\right) \varphi(x):=i\left[\hat{\mathcal{Q}}_{A}^{\mathrm{lat}}, \varphi(x)\right]_{(-1)^{|\varphi|+1}}, \\
\quad \text { or } \quad Q_{A}^{\mathrm{lat}} \varphi(x)=i T_{a_{A}}\left[\hat{\mathcal{Q}}_{A}^{\text {lat }}, \varphi(x)\right]_{(-1)^{|\varphi|+1}}
\end{gathered}
$$

It can also be written as

$$
\begin{aligned}
Q_{A}^{\text {lat }} \varphi(x) & =i T_{a_{A}} \hat{\mathcal{Q}}_{A}^{\text {lat }} \varphi(x)-(-1)^{|\varphi|} i T_{a_{A}} \varphi(x) T_{a_{A}}^{-1} T_{a_{A}} \hat{\mathcal{Q}}_{A}^{\text {lat }} \\
& =i \mathcal{Q}_{A}^{\text {lat }} \varphi(x)-(-1)^{|\varphi|} \varphi\left(x+a_{A}\right) i \mathcal{Q}_{A}^{\text {lat }}=: i\left[\mathcal{Q}_{A}^{\text {lat }}, \varphi(x)\right]_{(-1)^{|\varphi|+1}}^{\text {lat }}=: i \operatorname{ad}^{\text {lat }}\left(\mathcal{Q}_{A}^{\text {lat }}\right) \varphi(x),
\end{aligned}
$$

where $\mathcal{Q}_{A}^{\text {lat }}:=T_{a_{A}} \hat{\mathcal{Q}}_{A}^{\text {lat }}$. In this last equation we have defined a kind of deformed adjoint operation $\mathrm{ad}^{\text {lat }}$ which was referred to as the shifted (anti-)commutator in the DKKN formalism. It illustrates the general fact that an operator which obeys a modified Leibniz rule could be expressed with a shifted (anti-)commutator. We have, however, introduced objects like $\hat{\mathcal{Q}}_{A}^{\text {lat }}, \mathcal{Q}_{A}^{\text {lat }}$ and respectively their (anti-)commutator and shifted (anti-)commutator $\operatorname{ad}\left(\hat{\mathcal{Q}}_{A}^{\text {lat }}\right)$ and $\operatorname{ad}^{\text {lat }}\left(\mathcal{Q}_{A}^{\text {lat }}\right)$ only in a formal way, neither specified the explicit forms nor even justified the existence of them. So far we have only found that $T_{A}^{-1} Q_{A}^{\text {lat }}=\hat{Q}_{A}^{\text {lat }}$ obeys the usual Leibniz rule, which would be regarded as a normal operator, and that $Q_{A}^{\text {lat }}$ would be expressed as $Q_{A}^{\text {lat }}=T_{a_{A}} \hat{Q}_{A}^{\text {lat }}$. The point here is the following: As we mentioned above, we assume that the shift parameter $a_{A}$ reduces to zero in the naïve continuum limit. Correspondingly the translation/shift operator $T_{a_{A}}$ would go to unity in the limit: $T_{a_{A}} \rightarrow \mathbb{1}$, and thus the formal expression $Q_{A}^{\text {lat }}=i T_{a_{A}} \operatorname{ad}\left(\hat{\mathcal{Q}}_{A}^{\text {lat }}\right)=i$ ad ${ }^{\text {lat }}\left(\mathcal{Q}_{A}^{\text {lat }}\right)$ reduces to the normal (anti-)commutator $Q_{A}=i \operatorname{ad}\left(\mathcal{Q}_{A}\right)$. This implies that normal (anti-)commutators in the continuum, if used in any algebraic expressions, should be simply replaced with the shifted (anti-)commutators on the lattice to accommodate the modified Leibniz rule (2.11). This reminds us of the correspondence principle between the Poisson bracket in the classical theory and the commutator in the quantum theory. We are motivated by this analogy to think the lattice version of the superalgebra of a "quantization" of the continuum superalgebra. This viewpoint of the formulation is discussed in the next chapter.

Let us move on to the algebra (2.2). Here, for generality, we consider the modified Leibniz rule (2.15). The l.h.s. of the algebra applies on a product $\varphi_{1} \varphi_{2}$ as in

$$
\begin{aligned}
\left\{Q_{A}^{\mathrm{lat}}, Q_{B}^{\mathrm{lat}}\right\}\left(\varphi_{1} \varphi_{2}\right)(x) & =\left\{Q_{A}^{\mathrm{lat}}, Q_{B}^{\mathrm{lat}}\right\} \varphi_{1}(x) \varphi_{2}\left(x+a_{A}^{\mathrm{r}}+a_{B}^{\mathrm{r}}\right)+\varphi_{1}\left(x+a_{A}^{\mathrm{l}}+a_{B}^{\mathrm{l}}\right)\left\{Q_{A}^{\mathrm{lat}}, Q_{B}^{\mathrm{lat}}\right\} \varphi_{2}(x) \\
& =\sum_{\mu} 2 \tau_{A B}^{\mu}\left(P_{\mu}^{\mathrm{lat}} \varphi_{1}(x) \varphi_{2}\left(x+a_{A}^{\mathrm{r}}+a_{B}^{\mathrm{r}}\right)+\varphi_{1}\left(x+a_{A}^{\mathrm{l}}+a_{B}^{\mathrm{l}}\right) P_{\mu}^{\mathrm{lat}} \varphi_{2}(x)\right),
\end{aligned}
$$

while the r.h.s. as in

$$
2 \tau_{A B}^{\mu} P_{\mu}^{\mathrm{lat}}\left(\varphi_{1} \varphi_{2}\right)(x)=\sum_{\mu} 2 \tau_{A B}^{\mu}\left(P_{\mu}^{\mathrm{lat}} \varphi_{1}(x) \varphi_{2}\left(x+a^{\mathrm{r}} \hat{\mu}\right)+\varphi_{1}\left(x+a^{\mathrm{l}} \hat{\mu}\right) P_{\mu}^{\mathrm{lat}} \varphi_{2}(x)\right),
$$

where $a^{1}$ and $a^{\mathrm{r}}$ are, depending on the choice of $P_{\mu}^{\text {lat }}$,

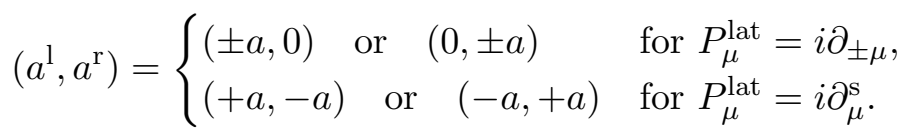

\footnotetext{
${ }^{4}$ Here in the equation below $[A, B]_{ \pm}:=A B \pm B A$.
} 
The algebra (2.2) requires that these two equations to be equal. As we can easily find, the first necessary condition is that the coefficient $\tau_{A B}^{\mu}$ should have the form

$$
\tau_{A B}^{\mu}=\tau_{A B} \delta_{\mu(A, B)}^{\mu}
$$

for a certain vector index $\mu(A, B)$ uniquely determined by the combination of spinor indices $A$ and $B$. Namely, only one, at most, of $D$ "momenta" $5 P_{1}^{\text {lat }}, \cdots, P_{D}^{\text {lat }}$ could appear in the r.h.s. of the algebra for each combination of $A$ and $B$. Then the corresponding algebra in the continuum would be such that $\left\{Q_{A}, Q_{B}\right\}=2 \tau_{A B} P_{\mu(A, B)}$, which violates the Lorentz covariance of the algebra except that $A$ or $B$ also has a "vector", or more precisely not just a spinor, index. We know such a basis of indices in which a supercharge has a "vector" index, namely as the basis of twisted supersymmetry [39] [10]. In fact in the link formalism and also in the other approaches the twisted basis for the spinor indices is adopted, and it is the twisted version of extended supersymmetry that the lattice formulations are constructed upon in those approaches. Here we see that the twist is necessary for the algebraic consistency in the link formalism, but the reason that twist, or extended supersymmetry itself from more general point of view, comes naturally into the lattice formulations is deeply connected to the doubling phenomenon on the lattice. Namely, the doubler's d.o.f., sometimes called "taste", is used as the $R$-symmetry "flavor" of the extended supersymmetry, and is put on the lattice in such a way that the theory becomes free from the mismatch of d.o.f. between fermions and bosons so that comes to meet the nonperturbative criterion for supersymmetry which reads that the partition function becomes unity. We will see this point again later.

At any rate suppose the coefficient $\tau_{A B}^{\mu}$ satisfies the condition (2.25). The condition that (2.22) coincides with (2.23) leads in this case to

$$
a_{A}^{\mathrm{l}}+a_{B}^{\mathrm{l}}=a^{\mathrm{l}} \hat{\mu}(A, B), \quad a_{A}^{\mathrm{r}}+a_{B}^{\mathrm{r}}=a^{\mathrm{r}} \hat{\mu}(A, B) .
$$

That these have consistent solutions for $a_{A}^{1, \mathrm{r}}$ is the second necessary condition for the link formalism to work. Recalling that for an operator $Q_{A}^{\text {lat }}$ which satisfies the modified Leibniz rule (2.15) the combination $T_{a_{A}^{1}}^{-1} Q_{A}^{\text {lat }} \overleftarrow{T}_{a_{A}^{\mathrm{r}}}$ follows the usual Leibniz rule in the adjoint representation, we may consider the corresponding algebra

$$
\left\{T_{a_{A}^{\mathrm{l}}}^{-1} Q_{A}^{\mathrm{lat}} \overleftarrow{T}_{a_{A}^{\mathrm{r}}}, T_{a_{B}^{\mathrm{l}}}^{-1} Q_{B}^{\mathrm{lat}} \overleftarrow{T}_{a_{B}^{\mathrm{r}}}\right\}=2 \tau_{A B} T_{a_{A}^{\mathrm{l}}}^{-1} T_{a_{B}^{\mathrm{l}}}^{-1} P_{\hat{\mu}(A, B)}^{\mathrm{lat}} \overleftarrow{T}_{a_{A}^{\mathrm{r}}} \overleftarrow{T}_{a_{B}^{\mathrm{r}}}
$$

where we have assumed that the condition (2.26) is met. The relation (2.26) assures that the operator in the r.h.s. also follows the usual Leibniz rule. In fact, we find

$$
T_{a_{A}^{\mathrm{l}}} T_{a_{B}^{\mathrm{l}}}=T_{a^{\mathrm{l}} \hat{\mu}(A, B)}, \quad T_{a_{A}^{\mathrm{r}}} T_{a_{B}^{\mathrm{r}}}=T_{a^{\mathrm{r}} \hat{\mu}(A, B)},
$$

and we may write $P_{\hat{\mu}(A, B)}^{\text {lat }}$ as, up to the lattice constant and other constant factors,

$$
\begin{aligned}
P_{\hat{\mu}(A, B)}^{\mathrm{lat}} & =\operatorname{Ad}\left(T_{a^{\mathrm{l}} \hat{\mu}(A, B)}\right)-\operatorname{Ad}\left(T_{a^{\mathrm{r}} \hat{\mu}(A, B)}\right), \\
\text { i.e. } \quad P_{\hat{\mu}(A, B)}^{\mathrm{lat}} \varphi(x) & =T_{a^{\mathrm{l}} \hat{\mu}(A, B)} \varphi(x) T_{a^{\mathrm{l}} \hat{\mu}(A, B)}^{-1}-T_{a^{\mathrm{r}} \hat{\mu}(A, B)} \varphi(x) T_{a^{\mathrm{r}} \hat{\mu}(A, B)}^{-1},
\end{aligned}
$$

where we define $\operatorname{Ad}\left(T_{a^{1} \hat{\mu}(A, B)}\right) \varphi(x)=T_{a^{1} \hat{\mu}(A, B)} \varphi(x) T_{a^{1} \hat{\mu}(A, B)}^{-1}$ which could be compared with the definition of $\operatorname{ad}\left(\hat{\mathcal{Q}}_{A}^{\text {lat }}\right)$ in (2.20). Then

$$
\begin{aligned}
T_{a_{A}^{1}}^{-1} T_{a_{B}^{1}}^{-1} P_{\hat{\mu}(A, B)}^{\mathrm{lat}} \overleftarrow{T}_{a_{A}^{\mathrm{r}}} \overleftarrow{T}_{a_{B}^{\mathrm{r}}} & =-\operatorname{ad}\left(T_{a^{1} \hat{\mu}(A, B)}^{-1} T_{a^{\mathrm{r}} \hat{\mu}(A, B)}\right), \\
\text { i.e. } \quad T_{a_{A}^{\mathrm{l}}}^{-1} T_{a_{B}^{\mathrm{l}}}^{-1} P_{\hat{\mu}(A, B)}^{\mathrm{lat}} \varphi(x) T_{a_{A}^{\mathrm{r}}} T_{a_{B}^{\mathrm{r}}} & =-\left(T_{a^{\mathrm{l}} \hat{\mu}(A, B)}^{-1} T_{a^{\mathrm{r}} \hat{\mu}(A, B)} \varphi(x)-\varphi(x) T_{a^{\mathrm{l}} \hat{\mu}(A, B)}^{-1} T_{a^{\mathrm{r}} \hat{\mu}(A, B)}\right)
\end{aligned}
$$

${ }^{5}$ We denote the spacetime dimension as $D$. 
which is a normal commutator. Notice that, as mentioned before, this redefinition of the "momentum" operator is possible only for $a_{A}^{\mathrm{r}} \neq a_{A}^{\mathrm{l}}$, which is assured here by the requirement that (2.26) holds. We have seen that all "generators" in the algebra (2.27) follow the usual Leibniz rule, so that would give a basis for the construction of supersymmetry on the lattice in a manner quite parallel to that of the continuum.

\subsection{Twisted Basis and the Doubling of Chiral Fermion}

When one regularizes chiral fermions on the lattice species doublers of chiral fermions inevitably appear [1, 2]. It was shown that the naïve fermion formulation where the continuum differential operators in the Dirac action is naïvely replaced by the lattice difference operator can be spin diagonalized and leads to the staggered fermion formulation [48] which is shown to be essentially equivalent [49, 50] to Kogut-Susskind fermion formulation [51]. The equivalence of the staggered fermion formulation and the Dirac-Kähler fermion has been proved exactly with an introduction of mild noncommutativity between differential forms and fields [16]. This means that all these lattice fermion formulations are equivalent where the mild noncommutativity seems to play an important rôle. Among these fermion formulations the Dirac-Kähler fermion formulation has clear geometrical correspondence with respect to the fields since the differential form and simplex of lattice have one to one correspondence.

The claim of the Dirac-Kähler twisting procedure is that these species doublers are not just lattice artifacts but fundamental d.o.f. for the regularization of fermions [10]. It is exactly these d.o.f. which constitute the twisted extended supersymmetry: $\mathcal{N}=2$ in two dimensions, $\mathcal{N}=4$ in three dimensions, and $\mathcal{N}=4$ in four dimensions. The four dimensional Dirac-Kähler twisting procedure coincides with the twisting derived by Marcus [11. These arguments apply in higher dimensions, too, requiring that in $D$ dimensions, which has $2^{D / 2}$ (on-shell) doubler's degeneracy , should be treated with $\mathcal{N}=2^{D / 2}$ extended supersymmetry. (In two dimensions $\mathcal{N}=2$, for example, does not correspond to the number of total charges and thus it is sometimes denoted as $\mathcal{N}=(2,2)$ instead.)

In the Dirac-Kähler twisting procedure spinor suffix and flavor suffix constitute the scalar, vector, tensor.. nature of the super charges. In other words the flavor d.o.f. which are originally the species doublers d.o.f. is now identified as the extended supersymmetry d.o.f.. The corresponding suffix can be rotated by the internal R-symmetry generator of extended supersymmetry. In this way the internal d.o.f. plays the rôle of changing spin of the fields. The mechanism how the spin and the internal rotation are related should be understood from lattice point of view. This issue is fundamentally related to the spin and statistics problem on the lattice. Since the Lorentz invariance is broken on the lattice it is natural to expect that the (anti-)commuting nature of fields will be modified.

Let us begin with the two dimensional case. Here we only consider the simplest cases. Superalgebra in the Dirac-Kähler twisted basis on the lattice is given as

$$
\left\{Q^{\text {lat }}, Q_{\mu}^{\text {lat }}\right\}=P_{\mu}^{\text {lat }}, \quad\left\{\tilde{Q}^{\text {lat }}, Q_{\mu}^{\text {lat }}\right\}=-\epsilon_{\mu \nu} P_{\nu}^{\text {lat'}}, \quad\{\text { others }\}=0,
$$

which is the twisted version of $\mathcal{N}=(2,2)$ superalgebra in two dimensions. We have put a prime on the second "momentum" operator to distinguish from the first one, since there is an ambiguity for the lattice "momentum" operator as explained above. Note in each commutator the r.h.s. contains only one "momentum" operator for each given combination of indices, which is necessary for the algebraic consistency as claimed in the preceding section. The reason we specified $\mathcal{N}=(2,2)$ is that then the corresponding supermultiplet contains four fermions, which has the same (on-shell) d.o.f. as that of the Dirac-Kähler/staggered fermions which originate the doubler's d.o.f. on the lattice in two dimensions. 
The shift variable condition (2.26) reads in this case

$$
a^{1, \mathrm{r}}+a_{\mu}^{1, \mathrm{r}}=a^{1, \mathrm{r}} \hat{\mu}, \quad \tilde{a}^{1, \mathrm{r}}+a_{\mu}^{1, \mathrm{r}}=\left|\epsilon_{\mu \nu}\right| a^{\prime 1, \mathrm{r}} \hat{\mu} .
$$

With the same argument given in the original link formalism these lead to $a^{1, \mathrm{r}}+a_{1}^{1, \mathrm{r}}+a_{2}^{1, \mathrm{r}}+\tilde{a}^{1, \mathrm{r}}=$ $\left(a^{l, \mathrm{r}}+a^{\prime l, \mathrm{r}}\right) \hat{1}=\left(a^{1, \mathrm{r}}+a^{\prime l, \mathrm{r}}\right) \hat{2}$, which is only possible if $a^{1, \mathrm{r}}=-a^{l, \mathrm{r}}$. In our simple choices of the "momentum" operators, it implies that, due to (2.24),

$$
\left\{\begin{array}{l}
P_{\mu}^{\text {lat }}=i \partial_{ \pm \mu}, \\
P_{\mu}^{\text {lat }}{ }_{\mu}=i \partial_{\mp \mu},
\end{array} \quad \text { or } \quad P_{\mu}^{\text {lat }}=P_{\mu}^{\text {lat }}{ }_{\mu}^{\prime}=i \partial_{\mu}^{\mathrm{s}} .\right.
$$

The former possibility was considered in the original link formulation, whereas the latter one, although a solution for the consistency, might not be so good from the viewpoint of the doubling issue: it would create, if naïvely used, the doubling degeneracy again. In any of these cases, the shift conditions become

$$
a^{1, \mathrm{r}}+a_{\mu}^{1, \mathrm{r}}=a^{1, \mathrm{r}} \hat{\mu}, \quad \tilde{a}^{1, \mathrm{r}}+a_{\mu}^{1, \mathrm{r}}=-\left|\epsilon_{\mu \nu}\right| a^{1, \mathrm{r}} \hat{\mu},
$$

which are four conditions with one constraint, so three remaining conditions in total, for four shift variables. It thus seems that one shift variable could be free. In view of the lattice structure, however, this free parameter should not be irrational, otherwise it would lead to uncountable number of "dual" lattice points, which spoils the lattice regularization! Though still any rational numbers are allowed, it is easy to see we will then have unnecessary d.o.f. again or unnatural lattice structure, except for the case when this free parameter is fixed to zero or half the lattice constant. These choices of the free parameter were referred to as the asymmetric and symmetric choices, respectively, in the link formalism.

Similarly in four dimensions, we take the superalgebra

$$
\begin{aligned}
& \left\{Q^{\text {lat }}, Q_{\mu}^{\text {lat }}\right\}=P_{+\mu}^{\text {lat }}, \quad\left\{Q_{\mu \nu}^{\text {lat }}, Q_{\rho}^{\text {lat }}\right\}=\delta_{\mu \nu, \rho \sigma} P_{-\sigma}^{\text {lat }}, \\
& \left\{\tilde{Q}^{\text {lat }}, \tilde{Q}_{\mu}^{\text {lat }}\right\}=P_{-\mu}^{\text {lat }}, \quad\left\{Q_{\mu \nu}^{\text {lat }}, \tilde{Q}_{\rho}^{\text {lat }}\right\}=\epsilon_{\mu \nu \rho \sigma} P_{+\sigma}^{\text {lat }}
\end{aligned}
$$

and the other commutators all vanish. This is the Dirac-Kähler twisted superalgebra of $\mathcal{N}=4$ which is required, as explained above, from the general argument on the fermionic d.o.f. We

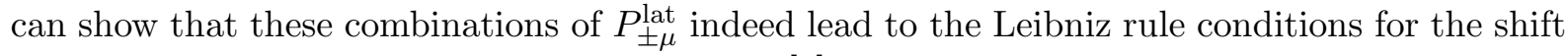
variables which have nontrivial set of solutions [7].

\subsection{The Claimed Inconsistency}

What is intriguing in the link formalism is the algebraic structure based on the modified Leibniz rule for the symmetry operators. If a suitable representation of this algebra is unambiguously obtained, it seems at first sight that it gives a formulation of supersymmetry on the lattice. It turns out, however, such a representation would conflict with the conventional component field path integral formulation on the lattice. This problem can be seen as the fact that, although supertransformations of single component fields are well-defined, supertransformations of products of fields becomes sensitive to the order of the fields in the products. If such an order is uniquely determined, it is nothing harmful. However, we have no criteria to introduce such an order on the conventional lattice, thus we have a serious difficulty that supertransformations are not totally defined in a unique and consistent manner as transformations of path integral variables. In fact, this difficulty is claimed as an inconsistency of the link formalism in [42]. The criticism is two-folded: one is for the non-gauge theories [6], the other is, also investigated in a similar attitude in [34], for the case of gauge theories [7, 8], and both are summarized as that the 
supercharges in the link formalism add nontrivial link structure on component fields changing the original link nature of the fields in an ordering sensitive way.

Let us see these arguments more explicitly. In the link formalism, scalar fields $\phi(x)$ defined on sites of the lattice are naturally assumed to be commutative

$$
\phi_{1}(x) \phi_{2}(x)=\phi_{2}(x) \phi_{1}(x)
$$

Applying the supertransformation on the both sides of this equation, we have, from the left hand side, that

$$
Q_{A}^{\mathrm{lat}}\left(\phi_{1}(x) \phi_{2}(x)\right)=\psi_{1 A}(x) \phi_{2}\left(x+a_{A}^{\mathrm{r}}\right)+\phi_{1}\left(x+a_{A}^{\mathrm{l}}\right) \psi_{2 A}(x),
$$

where $\psi_{1,2} A(x):=Q_{A}^{\text {lat }} \phi_{1,2}(x)$, and from the right,

$$
Q_{A}^{\mathrm{lat}}\left(\phi_{2}(x) \phi_{1}(x)\right)=\psi_{2 A}(x) \phi_{1}\left(x+a_{A}^{\mathrm{r}}\right)+\phi_{2}\left(x+a_{A}^{\mathrm{l}}\right) \psi_{1 A}(x)
$$

These two equations must be the same as they are the transformations of one and the same quantity; otherwise the supertransformations on products of fields aren't uniquely defined. But actually these two conflicts with each other if fermions $\psi_{1,2} A$ are also assumed to be simple (anti)commuting objects: the term containing $\psi_{1 A}(x)$ in the first equation has the factor $\phi_{2}\left(x+a_{A}^{\mathrm{r}}\right)$, whereas in the second has $\phi_{2}\left(x+a_{A}^{\mathrm{l}}\right)$, and they are different unless $a_{A}^{\mathrm{l}}=a_{A}^{\mathrm{r}}$, which, however, wouldn't lead to the consistent solution for the shift variable conditions as already explained in the previous sections. The discrepancy between these two equations cannot be expressed as a total difference, so that it gives an essential obstacle for the invariance of any possible action. It causes similar difficulties also in the gauge theory actions.

In the following chapters, we will propose a possible solution to the above mentioned first criticism for the non-gauge theories by introducing the following mild noncommutativity [8]:

$$
\varphi_{A}(x) \varphi_{B}(y)=(-1)^{\left|\varphi_{A}\right|\left|\varphi_{B}\right|} \varphi_{B}\left(y+a_{A}\right) \varphi_{A}\left(x-a_{B}\right),
$$

where $\varphi_{A}(x)$ and $\varphi_{B}(y)$ carry a shift $a_{A}$ and $a_{B}$, respectively. In fact we can easily confirm that the expressions of (2.37) and (2.38) coincide if we identify that $\psi_{1,2 A}$ carry a shift $a_{A}^{\mathrm{l}}-a_{A}^{\mathrm{r}}$ while $\phi_{1,2}$ carry no shift and they satisfy the noncommutative relation (2.39). The key point is to treat each field as a noncommutative object, or an object with nontrivial statistics, to uniquely define the ordering which is necessary to avoid the conflict.

If we introduce the noncommutative nature for the fields as in (2.39), the formulation of field theory should be modified from the conventional definition in such a way that any algebraic manipulation of fields and operators should be compatible with the new deformed supersymmetry. In the following we show that it is possible to define a new lattice field theory which has the exact deformed supersymmetry with Hopf algebraic nature. Addressing the similar questions to the gauge theories is out of the scope of this paper.

\section{Hopf Algebraic Structure of the Lattice Superalgebra}

In this section, we investigate the "lattice superalgebra" from a yet different algebraic viewpoint, namely in terms of Hopf algebra. As has been developed in recent years, extending the notion of the symmetry in a field theory to the Hopf algebraic one brings us still useful frameworks in some specific cases especially in noncommutative theories [41, 52, 53, 54, 55]. A slightly different applications are found in [16]. In the current interest, the superalgebra on the lattice is understood as a deformed algebra on the lattice and forming a Hopf algebra. This identification assures us of the mathematical consistency of the deformed algebra. Using the general scheme called braided quantum field theory [41, 54, we will show that the field theory whose symmetry 
is prescribed by the deformed algebra can be constructed at least perturbatively. The deformed symmetry leads to the corresponding Ward-Takahashi identities on the lattice, which may serve as a good physical interpretation of the deformed symmetry itself.

Appendix $\mathrm{A}$ is devoted to a brief mathematical basis on Hopf algebra and summarizing our notation and terminology.

\subsection{Lattice Superalgebra as a Hopf Algebra}

We begin with the lattice superalgebra (2.2), or those in the twisted basis (2.31), (2.35). Here we treat these as abstract Lie superalgebra and denote as $\mathcal{A}$, so that $P_{\mu}^{\text {lat }}, Q_{A}^{\text {lat }} \in \mathcal{A}$. We then introduce the space of fields on the lattice as $X=X_{\mathrm{e}} \oplus X_{\mathrm{o}}$, where $X_{\mathrm{e}}$ consists of all bosonic fields and $X_{\mathrm{o}}$ of all fermionic fields. We need a multiplication/product of fields to construct a field theory, which is in general noncommutative. We assume here this multiplication is associative for our current application. Thus the space $X$ is supposed to be an associative graded algebra. However, as a quantum field theory, products of fields, i.e. composite fields, could be clearly distinguished from the single fields, i.e. elementary fields, because the elementary fields are the variables of path integral (if any defined), or the ones obeying the canonical (anti-)commutation relations, whose behaviour is clearly different from that of the composite fields. We thus denote by $X$ the elementary fields and extend it to the formal space of all tensor products of the elementary fields to include any composite fields:

$$
\hat{X}:=\bigoplus_{n=0}^{\infty} X^{n}, \quad X^{0}:=X_{\mathrm{e}}^{0} \oplus X_{\mathrm{o}}^{0}, \quad X^{n}:=\underbrace{X \otimes \cdots \otimes X}_{n},
$$

where $X_{\mathrm{e}}^{0}$ and $X_{\mathrm{o}}^{0}$ are the space of bosonic and fermionic constant functions, respectively. Multiplications/products of fields are naturally defined in $\hat{X}$ as $m\left(\varphi \otimes \varphi^{\prime}\right)=\varphi \cdot \varphi^{\prime} \in \hat{X} \quad\left(\varphi, \varphi^{\prime} \in \hat{X}\right)$.

We now consider general action (see Appendix A) of $\mathcal{A}$ on the space of fields $\hat{X}$. We denote the action of an operator $a \in \mathcal{A}$ as $a \triangleright$. With the successive actions, we are naturally led to the notion of an (associative) universal enveloping algebra $\mathcal{U}(\mathcal{A})$ of $\mathcal{A}$, as in $(a \cdot b) \triangleright:=a \triangleright \circ b \triangleright:=$ $a \triangleright(b \triangleright)$, with $a, b \in \mathcal{A}$ and $a \cdot b \in \mathcal{U}(\mathcal{A})$. We also introduce the identity operator $\mathbb{1}$ as a unit element of the universal enveloping algebra. We may define the unit map by $\eta(c):=c \mathbb{1}, c \in \mathbb{C}$.

Even on the lattice, actions or representations of the operators $Q_{A}^{\text {lat }}$ and $P_{\mu}^{\text {lat }}$ on elementary fields would be well-defined with no difficulty. We denote these formally as

$$
Q_{A}^{\text {lat }} \triangleright \varphi(x)=\left(Q_{A}^{\text {lat }} \varphi\right)(x), \quad P_{\mu}^{\text {lat }} \triangleright \varphi(x)=\left(P_{\mu}^{\text {lat }} \varphi\right)(x), \quad \varphi \in X .
$$

Explicit form of $Q_{A}^{\text {lat }} \varphi$ depends on the model we take. An example is listed in the appendix B. As for the expression $P_{\mu}^{\text {lat }} \varphi$, we could essentially take some of the difference operators as in (2.5), but it turns out that lattice momentum operator $P_{\mu}^{\text {lat }}$ should carry a nontrivial grading structure, which is required from the Hopf algebraic consistency. We will see this point in the following subsection.

Actions on the trivial/constant fields are also easily defined as in

$$
Q_{A}^{\text {lat }} \triangleright f=0, \quad P_{\mu}^{\text {lat }} \triangleright f=0, \quad f \in X_{\mathrm{e}}^{0} .
$$

As a matter of convention, we write these equations in terms of a map $\epsilon$ called counit as in

$$
\begin{array}{lll}
Q_{A}^{\text {lat }} \triangleright f=\epsilon\left(Q_{A}^{\text {lat }}\right) f=0, & \text { i.e. } & \epsilon\left(Q_{A}^{\text {lat }}\right)=0, \\
P_{\mu}^{\text {lat }} \triangleright f=\epsilon\left(P_{\mu}^{\text {lat }}\right) f=0, & \text { i.e. } & \epsilon\left(P_{\mu}^{\text {lat }}\right)=0 .
\end{array}
$$

The essential nontriviality comes in the actions of the operators on composite fields, i.e. product of the elementary fields, due to the failure of the usual Leibniz rule. The link formalism manages this difficulty with the introduction of appropriate deformation or modification of 
Leibniz rules when the operators act on the composite fields. Mathematically, this is understood as equipping the universal enveloping algebra $\mathcal{U}(\mathcal{A})$ with an additional structure, the coproduct/comultiplication, denoted by $\Delta$. To be specific, consider the actions of $Q_{A}^{\text {lat }}$ and $P_{\mu}^{\text {lat }}$ on a product of two elementary fields $\varphi_{1}(x), \varphi_{2}(x) \in X$. Introducing the modified Leibniz rule (2.15) and (2.23) is equivalent to defining these actions to be

$$
\begin{aligned}
& Q_{A}^{\text {lat }} \triangleright\left(\varphi_{1}(x) \cdot \varphi_{2}(x)\right):=m\left(\Delta\left(Q_{A}^{\text {lat }}\right) \triangleright\left(\varphi_{1}(x) \otimes \varphi_{2}(x)\right)\right), \\
& P_{\mu}^{\text {lat }} \triangleright\left(\varphi_{1}(x) \cdot \varphi_{2}(x)\right):=m\left(\Delta\left(P_{\mu}^{\text {lat }}\right) \triangleright\left(\varphi_{1}(x) \otimes \varphi_{2}(x)\right)\right),
\end{aligned}
$$

together with the coproducts

$$
\begin{aligned}
& \Delta\left(Q_{A}^{\mathrm{lat}}\right)=Q_{A}^{\mathrm{lat}} \otimes T_{a_{A}^{\mathrm{r}}}+(-1)^{\mathcal{F}} \cdot T_{a_{A}^{\mathrm{l}}} \otimes Q_{A}^{\mathrm{lat}}, \\
& \Delta\left(P_{\mu}^{\mathrm{lat}}\right)=P_{\mu}^{\mathrm{lat}} \otimes T_{a^{\mathrm{r}} \hat{\mu}}+T_{a^{1} \hat{\mu}} \otimes P_{\mu}^{\mathrm{lat}},
\end{aligned}
$$

where $\mathcal{F}$ is the fermion number operator with which $(-1)^{\mathcal{F}}$ takes care of the statistics factors, and the shift operator $T_{b}$, which is also assumed to belong to $\mathcal{U}(\mathcal{A})$, acts as

$$
T_{b} \triangleright \varphi(x):=\varphi(x+b) .
$$

For these operators we set

$$
\epsilon\left(T_{b}\right)=1, \quad \Delta\left(T_{b}\right)=T_{b} \otimes T_{b},
$$

and

$$
\epsilon\left((-1)^{\mathcal{F}}\right)=1, \quad \Delta\left((-1)^{\mathcal{F}}\right)=(-1)^{\mathcal{F}} \otimes(-1)^{\mathcal{F}} .
$$

Note that these definitions are natural, since the counit essentially prescribes the action on a constant, whereas the coproduct defines the action on a product. We also list, though obvious, the action of the identity operator $\mathbb{1}$ on $\hat{X}$. It must be, by definition, such that $\mathbb{1} \triangleright \varphi=\varphi, \varphi \in X$. On a constant field, $f=\mathbb{1} \triangleright f=\epsilon(\mathbb{1}) f, f \in X^{0}$, so that

$$
\epsilon(\mathbb{1})=1 \text {. }
$$

On a product of elementary fields, $\varphi_{1} \cdot \varphi_{2}=\mathbb{1} \triangleright\left(\varphi_{1} \cdot \varphi_{2}\right)=m\left(\Delta(\mathbb{1}) \triangleright\left(\varphi_{1} \otimes \varphi_{2}\right)\right)$, so that

$$
\Delta(\mathbb{1})=\mathbb{1} \otimes \mathbb{1} .
$$

Counit $\epsilon$ and coproduct $\Delta$ has to satisfy some consistency conditions. First, we note that any single elementary field $\varphi$ might be expressed as a product of unity and itself; $\varphi=m(1 \otimes \varphi)=$ $m(\varphi \otimes 1)$. The action should be uniquely determined regardless of this reinterpretation of the degrees of product. More specifically, this requires that

$$
\begin{aligned}
\left(Q_{A}^{\mathrm{lat}} \varphi\right)(x)=Q_{A}^{\mathrm{lat}} \triangleright \varphi(x) & =Q_{A}^{\mathrm{lat}} \triangleright m(1 \otimes \varphi(x))=m\left(\Delta\left(Q_{A}^{\mathrm{lat}}\right) \triangleright(1 \otimes \varphi(x))\right) \\
& =m\left(\left(Q_{A}^{\mathrm{lat}} \triangleright 1\right) \otimes\left(T_{a_{A}^{\mathrm{r}}} \triangleright \varphi(x)\right)+\left(T_{a_{A}^{1}} \triangleright 1\right) \otimes\left(Q_{A}^{\mathrm{lat}} \triangleright \varphi(x)\right)\right) \\
& =m\left(\left(\epsilon\left(Q_{A}^{\mathrm{lat}}\right) 1\right) \otimes\left(T_{a_{A}^{\mathrm{r}}} \triangleright \varphi(x)\right)+\left(\epsilon\left(T_{a_{A}^{1}}\right) 1\right) \otimes\left(Q_{A}^{\mathrm{lat}} \triangleright \varphi(x)\right)\right) \\
& =m\left(1 \otimes\left(Q_{A}^{\mathrm{lat}} \varphi\right)(x)\right)=\left(Q_{A}^{\mathrm{lat}} \varphi\right)(x),
\end{aligned}
$$

which is consistently realized. The other consistency also holds:

$$
\begin{aligned}
\left(Q_{A}^{\mathrm{lat}} \varphi\right)(x)=Q_{A}^{\mathrm{lat}} \triangleright \varphi(x) & =Q_{A}^{\mathrm{lat}} \triangleright m(\varphi(x) \otimes 1)=m\left(\Delta\left(Q_{A}^{\mathrm{lat}}\right) \triangleright(\varphi(x) \otimes 1)\right) \\
& =m\left(\left(Q_{A}^{\mathrm{lat}} \triangleright \varphi(x)\right) \otimes\left(T_{a_{A}^{\mathrm{r}}} \triangleright 1\right)+\left((-1)^{\mathcal{F}} \cdot T_{a_{A}^{\mathrm{l}}} \triangleright \varphi(x)\right) \otimes\left(Q_{A}^{\mathrm{lat}} \triangleright 1\right)\right) \\
& =m\left(\left(Q_{A}^{\mathrm{lat}} \triangleright \varphi(x)\right) \otimes\left(\epsilon\left(T_{a_{A}^{\mathrm{r}}}\right) 1\right)+\left((-1)^{|\varphi|} T_{a_{A}^{\mathrm{l}}} \triangleright \varphi(x)\right) \otimes\left(\epsilon\left(Q_{A}^{\mathrm{lat}}\right) 1\right)\right) \\
& =m\left(\left(Q_{A}^{\mathrm{lat}} \varphi\right)(x) \otimes 1\right)=\left(Q_{A}^{\mathrm{lat}} \varphi\right)(x) .
\end{aligned}
$$


Similar results hold for $P_{\mu}^{\text {lat }}$. As for $T_{b}$,

$$
\begin{aligned}
\varphi(x+b)=T_{b} \triangleright \varphi(x) & =T_{b} \triangleright m(1 \otimes \varphi(x))=m\left(\Delta\left(T_{b}\right) \triangleright(1 \otimes \varphi(x))\right) \\
& =m\left(\left(T_{b} \triangleright 1\right) \otimes\left(T_{b} \triangleright \varphi(x)\right)\right) \\
& =m\left(\left(\epsilon\left(T_{b}\right) 1\right) \otimes \varphi(x+b)\right)=m(1 \otimes \varphi(x+b))=\varphi(x+b),
\end{aligned}
$$

which is again consistent. These result show that the definitions of counit and coproduct in (3.4), (3.6), (3.8) are compatible to the trivial unital structure of the algebra $\hat{X}$. Second consistency condition is so-called the coassociativity. Since the multiplication on $\hat{X}$ is associative, actions on products of three elementary fields should respect this associativity. This requires the coassociativity for the coproduct. It also means the action on products of three elementary fields is defined in a natural way as in

$$
\begin{aligned}
& m \circ(m \otimes \mathrm{id}) \circ(\Delta \otimes \mathrm{id}) \circ \Delta\left(Q_{A}^{\mathrm{lat}}\right) \triangleright\left(\left(\varphi_{1}(x) \otimes \varphi_{2}(x)\right) \otimes \varphi_{3}(x)\right) \\
& =Q_{A}^{\mathrm{lat}} \triangleright\left(\left(\varphi_{1}(x) \cdot \varphi_{2}(x)\right) \cdot \varphi_{3}(x)\right) \\
& =Q_{A}^{\mathrm{lat}} \triangleright\left(\varphi_{1}(x) \cdot \varphi_{2}(x) \cdot \varphi_{3}(x)\right) \\
& =Q_{A}^{\mathrm{lat}} \triangleright\left(\varphi_{1}(x) \cdot\left(\varphi_{2}(x) \cdot \varphi_{3}(x)\right)\right) \\
& =m \circ(\mathrm{id} \otimes m) \circ(\mathrm{id} \otimes \Delta) \circ \Delta\left(Q_{A}^{\mathrm{lat}}\right) \triangleright\left(\varphi_{1}(x) \otimes\left(\varphi_{2}(x) \otimes \varphi_{3}(x)\right)\right) .
\end{aligned}
$$

Since the product $m$ is associative,

$$
m \circ(m \otimes \mathrm{id})=m \circ(\mathrm{id} \otimes m),
$$

it requires that

$$
(\Delta \otimes \mathrm{id}) \circ \Delta\left(Q_{A}^{\text {lat }}\right)=(\mathrm{id} \otimes \Delta) \circ \Delta\left(Q_{A}^{\text {lat }}\right) .
$$

The same condition should follow for $P_{\mu}^{\text {lat }}$ and $T_{b}$. These conditions are indeed satisfied for the coproducts in the present case. Using (3.6), we compute 6

$$
\begin{aligned}
& (\Delta \otimes \mathrm{id}) \circ \Delta\left(Q_{A}^{\mathrm{lat}}\right)=(\Delta \otimes \mathrm{id})\left(Q_{A}^{\text {lat }} \otimes T_{a_{A}^{\mathrm{r}}}+(-1)^{\mathcal{F}} \cdot T_{a_{A}^{1}} \otimes Q_{A}^{\text {lat }}\right) \\
& =\left(Q_{A}^{\text {lat }} \otimes T_{a_{A}^{\mathrm{r}}}+(-1)^{\mathcal{F}} \cdot T_{a_{A}^{1}} \otimes Q_{A}^{\text {lat }}\right) \otimes T_{a_{A}^{\mathrm{r}}}+\left((-1)^{\mathcal{F}} \cdot T_{a_{A}^{1}} \otimes(-1)^{\mathcal{F}} \cdot T_{a_{A}^{1}}\right) \otimes Q_{A}^{\text {lat }} \\
& =Q_{A}^{\text {lat }} \otimes T_{a_{A}^{\mathrm{r}}} \otimes T_{a_{A}^{\mathrm{r}}}+(-1)^{\mathcal{F}} \cdot T_{a_{A}^{1}} \otimes Q_{A}^{\text {lat }} \otimes T_{a_{A}^{\mathrm{r}}}+(-1)^{\mathcal{F}} \cdot T_{a_{A}^{1}} \otimes(-1)^{\mathcal{F}} \cdot T_{a_{A}^{1}} \otimes Q_{A}^{\text {lat }},
\end{aligned}
$$

and

$$
\begin{aligned}
& (\mathrm{id} \otimes \Delta) \circ \Delta\left(Q_{A}^{\mathrm{lat}}\right)=(\mathrm{id} \otimes \Delta)\left(Q_{A}^{\mathrm{lat}} \otimes T_{a_{A}^{\mathrm{r}}}+(-1)^{\mathcal{F}} \cdot T_{a_{A}^{\mathrm{l}}} \otimes Q_{A}^{\mathrm{lat}}\right) \\
& =Q_{A}^{\mathrm{lat}} \otimes\left(T_{a_{A}^{\mathrm{r}}} \otimes T_{a_{A}^{\mathrm{r}}}\right)+(-1)^{\mathcal{F}} \cdot T_{a_{A}^{\mathrm{l}}} \otimes\left(Q_{A}^{\mathrm{lat}} \otimes T_{a_{A}^{\mathrm{r}}}+(-1)^{\mathcal{F}} \cdot T_{a_{A}^{1}} \otimes Q_{A}^{\mathrm{lat}}\right) \\
& =Q_{A}^{\mathrm{lat}} \otimes T_{a_{A}^{\mathrm{r}}} \otimes T_{a_{A}^{\mathrm{r}}}+(-1)^{\mathcal{F}} \cdot T_{a_{A}^{1}} \otimes Q_{A}^{\mathrm{lat}} \otimes T_{a_{A}^{\mathrm{r}}}+(-1)^{\mathcal{F}} \cdot T_{a_{A}^{1}} \otimes(-1)^{\mathcal{F}} \cdot T_{a_{A}^{1}} \otimes Q_{A}^{\mathrm{lat}},
\end{aligned}
$$

which shows that (3.17) holds for $Q_{A}^{\text {lat }}$. We have thus found unambiguously that

$$
\begin{aligned}
& Q_{A}^{\mathrm{lat}} \triangleright\left(\varphi_{1}(x) \cdot \varphi_{2}(x) \cdot \varphi_{3}(x)\right) \\
& =\left(Q_{A}^{\mathrm{lat}} \varphi_{1}\right)(x) \cdot \varphi_{2}\left(x+a_{A}^{\mathrm{r}}\right) \cdot \varphi_{3}\left(x+a_{A}^{\mathrm{r}}\right) \\
& \quad+(-1)^{\left|\varphi_{1}\right|} \varphi_{1}\left(x+a_{A}^{\mathrm{l}}\right) \cdot\left(Q_{A}^{\mathrm{lat}} \varphi_{2}\right)(x) \cdot \varphi_{3}\left(x+a_{A}^{\mathrm{r}}\right) \\
& \quad+(-1)^{\left|\varphi_{1}\right|+\left|\varphi_{2}\right|} \varphi_{1}\left(x+a_{A}^{\mathrm{l}}\right) \cdot \varphi_{2}\left(x+a_{A}^{\mathrm{l}}\right) \cdot\left(Q_{A}^{\mathrm{lat}} \varphi_{3}\right)(x) .
\end{aligned}
$$

\footnotetext{
${ }^{6}$ Here we use the relation $\Delta\left((-1)^{\mathcal{F}} \cdot T_{b}\right)=\Delta\left((-1)^{\mathcal{F}}\right) \cdot \Delta\left(T_{b}\right)$, which will be explained shortly.
} 
The same is true for $P_{\mu}^{\text {lat: }}$

$$
\begin{aligned}
& P_{\mu}^{\text {lat }} \triangleright\left(\varphi_{1}(x) \cdot \varphi_{2}(x) \cdot \varphi_{3}(x)\right) \\
& =\left(P_{\mu}^{\operatorname{lat}} \varphi_{1}\right)(x) \cdot \varphi_{2}\left(x+a^{\mathrm{r}} \hat{\mu}\right) \cdot \varphi_{3}\left(x+a^{\mathrm{r}} \hat{\mu}\right) \\
& \quad+\varphi_{1}\left(x+a^{\mathrm{l}} \hat{\mu}\right) \cdot\left(P_{\mu}^{\mathrm{lat}} \varphi_{2}\right)(x) \cdot \varphi_{3}\left(x+a^{\mathrm{r}} \hat{\mu}\right) \\
& \quad+\varphi_{1}\left(x+a^{\mathrm{l}} \hat{\mu}\right) \cdot \varphi_{2}\left(x+a^{\mathrm{l}} \hat{\mu}\right) \cdot\left(P_{\mu}^{\mathrm{lat}} \varphi_{3}\right)(x) .
\end{aligned}
$$

Similarly, $T_{b}$ satisfies the coassociativity, for

$$
\begin{aligned}
(\Delta \otimes \mathrm{id}) \circ \Delta\left(T_{b}\right) & =(\Delta \otimes \mathrm{id})\left(T_{b} \otimes T_{b}\right)=\left(T_{b} \otimes T_{b}\right) \otimes T_{b} \\
& =T_{b} \otimes\left(T_{b} \otimes T_{b}\right)=(\mathrm{id} \otimes \Delta)\left(T_{b} \otimes T_{b}\right)=(\mathrm{id} \otimes \Delta) \Delta\left(T_{b}\right),
\end{aligned}
$$

so that

$$
T_{b} \triangleright\left(\varphi_{1}(x) \cdot \varphi_{2}(x) \cdot \varphi_{3}(x)\right)=\varphi_{1}(x+b) \cdot \varphi_{2}(x+b) \cdot \varphi_{3}(x+b) .
$$

The result for $(-1)^{\mathcal{F}}$ would be obvious.

Now that we have shown that our operators of the prime interest, $Q_{A}^{\text {lat }}, P_{\mu}^{\text {lat }}, T_{b}$ are well defined concerning to the actions on the elementary fields, constants, and products of two or three elementary fields, we find that any other actions are also consistently defined (needless to say actions as well as the maps introduced above are all linear). In particular the actions on any numbers of elementary fields can be computed inductively using the coassociativity. We need further the actions of products of operators. As we started above, the product of operators is defined as an operator of the successive applications of each operator in the product. On the elementary fields, it is easily understood, because it is nothing but the definition. On the trivial (i.e. constant) fields, this implies a consistency on the counit map, as

$$
\epsilon(a \cdot b) f=(a \cdot b) \triangleright f=a \triangleright \circ b \triangleright f=\epsilon(a) \epsilon(b) f,
$$

i.e.

$$
\epsilon(a \cdot b)=\epsilon(a) \epsilon(b) .
$$

Similarly, the product of operators should act on a product of elementary fields with the successive operations with

$$
\begin{aligned}
m\left(\Delta(a \cdot b) \triangleright\left(\varphi_{1} \otimes \varphi_{2}\right)\right) & =(a \cdot b) \triangleright\left(\varphi_{1} \cdot \varphi_{2}\right)=a \triangleright \circ b \triangleright\left(\varphi_{1} \cdot \varphi_{2}\right)=a \triangleright m\left(\Delta(b) \triangleright\left(\varphi_{1} \otimes \varphi_{2}\right)\right) \\
& =m\left(\Delta(a) \triangleright \Delta(b) \triangleright\left(\varphi_{1} \otimes \varphi_{2}\right)\right)=m\left((\Delta(a) \cdot \Delta(b)) \triangleright\left(\varphi_{1} \otimes \varphi_{2}\right)\right),
\end{aligned}
$$

so implies

$$
\Delta(a \cdot b)=\Delta(a) \cdot \Delta(b) .
$$

As an example, we compute

$$
\begin{aligned}
\Delta\left(Q_{A}^{\mathrm{lat}} \cdot Q_{B}^{\mathrm{lat}}\right) & =\Delta\left(Q_{A}^{\mathrm{lat}}\right) \cdot \Delta\left(Q_{B}^{\mathrm{lat}}\right) \\
& =\left(Q_{A}^{\mathrm{lat}} \otimes T_{a_{A}^{\mathrm{r}}}+(-1)^{\mathcal{F}} \cdot T_{a_{A}^{\mathrm{l}}} \otimes Q_{A}^{\mathrm{lat}}\right) \cdot\left(Q_{B}^{\mathrm{lat}} \otimes T_{a_{B}^{\mathrm{r}}}+(-1)^{\mathcal{F}} \cdot T_{a_{B}^{\mathrm{l}}} \otimes Q_{B}^{\mathrm{lat}}\right) \\
& =Q_{A}^{\mathrm{lat}} \cdot Q_{B}^{\mathrm{lat}} \otimes T_{a_{A}^{\mathrm{r}}} \cdot T_{a_{B}^{\mathrm{r}}}+Q_{A}^{\mathrm{lat}} \cdot(-1)^{\mathcal{F}} \cdot T_{a_{B}^{\mathrm{l}}} \otimes T_{a_{A}^{\mathrm{r}}} \cdot Q_{B}^{\mathrm{lat}} \\
& +(-1)^{\mathcal{F}} \cdot T_{a_{A}^{1}} \cdot Q_{B}^{\mathrm{lat}} \otimes Q_{A}^{\mathrm{lat}} \cdot T_{a_{B}^{\mathrm{r}}}+(-1)^{\mathcal{F}} \cdot T_{a_{A}^{1}} \cdot(-1)^{\mathcal{F}} \cdot T_{a_{B}^{\mathrm{l}}} \otimes Q_{A}^{\mathrm{lat}} \cdot Q_{B}^{\mathrm{lat}} .
\end{aligned}
$$


Other simple examples are

$$
\begin{aligned}
\Delta\left(Q_{A}^{\mathrm{lat}} \cdot P_{\mu}^{\mathrm{lat}}\right) & =\Delta\left(Q_{A}^{\mathrm{lat}}\right) \cdot \Delta\left(P_{\mu}^{\mathrm{lat}}\right) \\
& =\left(Q_{A}^{\mathrm{lat}} \otimes T_{a_{A}^{\mathrm{r}}}+(-1)^{\mathcal{F}} \cdot T_{a_{A}^{\mathrm{l}}} \otimes Q_{A}^{\mathrm{lat}}\right) \cdot\left(P_{\mu}^{\mathrm{lat}} \otimes T_{a^{\mathrm{r}} \hat{\mu}}+T_{a^{\mathrm{l}} \hat{\mu}} \otimes P_{\mu}^{\mathrm{lat}}\right) \\
& =Q_{A}^{\mathrm{lat}} \cdot P_{\mu}^{\mathrm{lat}} \otimes T_{a_{A}^{\mathrm{r}}} \cdot T_{a^{\mathrm{r}} \hat{\mu}}+Q_{A}^{\mathrm{lat}} \cdot T_{a^{\mathrm{l}} \hat{\mu}} \otimes T_{a_{A}^{\mathrm{r}}} \cdot P_{\mu}^{\mathrm{lat}} \\
& +(-1)^{\mathcal{F}} \cdot T_{a_{A}^{1}} \cdot P_{\mu}^{\mathrm{lat}} \otimes Q_{A}^{\mathrm{lat}} \cdot T_{a^{\mathrm{r}} \hat{\mu}}+(-1)^{\mathcal{F}} \cdot T_{a_{A}^{\mathrm{l}}} \cdot T_{a^{\mathrm{l}} \hat{\mu}} \otimes Q_{A}^{\mathrm{lat}} \cdot P_{\mu}^{\mathrm{lat}},
\end{aligned}
$$

and

$$
\begin{gathered}
\Delta\left(T_{b} \cdot Q_{A}^{\mathrm{lat}}\right)=\Delta\left(T_{b}\right) \cdot \Delta\left(Q_{A}^{\mathrm{lat}}\right)=\left(T_{b} \otimes T_{b}\right) \cdot\left(Q_{A}^{\mathrm{lat}} \otimes T_{a_{A}^{\mathrm{r}}}+(-1)^{\mathcal{F}} \cdot T_{a_{A}^{\mathrm{l}}} \otimes Q_{A}^{\mathrm{lat}}\right) \\
=T_{b} \cdot Q_{A}^{\mathrm{lat}} \otimes T_{b} \cdot T_{a_{A}^{\mathrm{r}}}+T_{b} \cdot(-1)^{\mathcal{F}} \cdot T_{a_{A}^{\mathrm{l}}} \otimes T_{b} \cdot Q_{A}^{\mathrm{lat}}, \\
\Delta\left(T_{b} \cdot T_{c}\right)=\Delta\left(T_{b}\right) \cdot \Delta\left(T_{c}\right)=\left(T_{b} \otimes T_{b}\right) \cdot\left(T_{c} \otimes T_{c}\right)=T_{b} \cdot T_{c} \otimes T_{b} \cdot T_{c} .
\end{gathered}
$$

Let us recall now the superalgebra (2.2), and introduce a natural algebra with respect to $T_{b}$ as in

$$
\begin{gathered}
\left\{Q_{A}^{\text {lat }}, Q_{B}^{\text {lat }}\right\}=2 \tau_{A B}^{\mu} P_{\mu}^{\text {lat }}, \\
{\left[Q_{A}^{\text {lat }}, P_{\mu}^{\text {lat }}\right]=\left[P_{\mu}^{\text {lat }}, P_{\nu}^{\text {lat }}\right]=0,} \\
{\left[Q_{A}^{\text {lat }}, T_{b}\right]=\left[P_{\mu}^{\text {lat }}, T_{b}\right]=\left[T_{b}, T_{c}\right]=0 .}
\end{gathered}
$$

The last relations are in a way obvious, and states that

$$
Q_{A}^{\mathrm{lat}} \varphi(x+b)=T_{b}\left(Q_{A}^{\mathrm{lat}} \varphi\right)(x), \quad T_{b} \varphi(x+c)=T_{c} \varphi(x+b)=\varphi(x+b+c)=T_{b+c} \varphi(x),
$$

and similar for $P_{\mu}^{\text {lat }}$. We also list here the obvious algebra for $(-1)^{\mathcal{F}}$ :

$$
\left\{Q_{A}^{\text {lat }},(-1)^{\mathcal{F}}\right\}=\left[P_{A}^{\text {lat }},(-1)^{\mathcal{F}}\right]=\left[T_{b},(-1)^{\mathcal{F}}\right]=0, \quad(-1)^{\mathcal{F}} \cdot(-1)^{\mathcal{F}}=\mathbb{1} .
$$

From these relations and using (3.28), we find that

$$
\Delta\left(\left\{Q_{A}^{\text {lat }}, Q_{B}^{\text {lat }}\right\}\right)=\left\{Q_{A}^{\text {lat }}, Q_{B}^{\text {lat }}\right\} \otimes T_{a_{A}^{\mathrm{r}}} \cdot T_{a_{B}^{\mathrm{r}}}+T_{a_{A}^{\mathrm{l}}} \cdot T_{a_{B}^{\mathrm{l}}} \otimes\left\{Q_{A}^{\text {lat }}, Q_{B}^{\text {lat }}\right\},
$$

reproducing the general result we found in (2.22). Just as an additional explicit check of the consistency, we compute the action of the product $Q_{A}^{\text {lat }} \cdot Q_{B}^{\text {lat }}$ on the product of three fields $\varphi_{1} \cdot \varphi_{2} \cdot \varphi_{3}$, which is given by the object

$$
\begin{aligned}
(\Delta \otimes \mathrm{id}) \circ \Delta\left(Q_{A}^{\mathrm{lat}} \cdot Q_{B}^{\mathrm{lat}}\right) & =(\Delta \otimes \mathrm{id}) \circ\left(\Delta\left(Q_{A}^{\mathrm{lat}}\right) \cdot \Delta\left(Q_{B}^{\mathrm{lat}}\right)\right) \\
& =\Delta\left(Q_{A}^{\mathrm{lat}} \cdot Q_{B}^{\mathrm{lat}}\right) \otimes T_{a_{A}^{\mathrm{r}}} \cdot T_{a_{B}^{\mathrm{r}}}+\Delta\left(Q_{A}^{\mathrm{lat}} \cdot(-1)^{\mathcal{F}} \cdot T_{a_{B}^{\mathrm{l}}}\right) \otimes T_{a_{A}^{\mathrm{r}}} \cdot Q_{B}^{\text {lat }} \\
& -\Delta\left(Q_{B}^{\mathrm{lat}} \cdot(-1)^{\mathcal{F}} \cdot T_{a_{A}^{\mathrm{l}}}\right) \otimes Q_{A}^{\mathrm{lat}} \cdot T_{a_{B}^{\mathrm{r}}}+\Delta\left(T_{a_{A}^{1}} \cdot T_{a_{B}^{1}}\right) \otimes Q_{A}^{\text {lat }} \cdot Q_{B}^{\text {lat }}
\end{aligned}
$$

and then this can be computed using (3.28), (3.30) and (3.31). This of course leads to

$$
\begin{aligned}
(\Delta \otimes \mathrm{id}) \circ \Delta\left(\left\{Q_{A}^{\mathrm{lat}}, Q_{B}^{\mathrm{lat}}\right\}\right) & =(\Delta \otimes \mathrm{id}) \circ\left(\left\{\Delta\left(Q_{A}^{\mathrm{lat}}\right), \Delta\left(Q_{B}^{\mathrm{lat}}\right)\right\}\right) \\
& =\Delta\left(\left\{Q_{A}^{\mathrm{lat}}, Q_{B}^{\mathrm{lat}}\right\}\right) \otimes T_{a_{A}^{\mathrm{r}}} \cdot T_{a_{B}^{\mathrm{r}}}+\Delta\left(T_{a_{A}^{1}} \cdot T_{a_{B}^{\mathrm{l}}}\right) \otimes\left\{Q_{A}^{\text {lat }}, Q_{B}^{\text {lat }}\right\} \\
& =\left\{Q_{A}^{\mathrm{lat}}, Q_{B}^{\mathrm{lat}}\right\} \otimes T_{a_{A}^{\mathrm{r}}} \cdot T_{a_{B}^{\mathrm{r}}} \otimes T_{a_{A}^{\mathrm{r}}} \cdot T_{a_{B}^{\mathrm{r}}} \\
& +T_{a_{A}^{1}} \cdot T_{a_{B}^{1}} \otimes\left\{Q_{A}^{\text {lat }}, Q_{B}^{\mathrm{lat}}\right\} \otimes T_{a_{A}^{\mathrm{r}}} \cdot T_{a_{B}^{\mathrm{r}}} \\
& +T_{a_{A}^{1}} \cdot T_{a_{B}^{\mathrm{l}}} \otimes T_{a_{A}^{1}} \cdot T_{a_{B}^{\mathrm{l}}} \otimes\left\{Q_{A}^{\text {lat }}, Q_{B}^{\text {lat }}\right\} .
\end{aligned}
$$

Equations (3.10), (3.25) and (3.11), (3.27) naturally require that the counit and coproduct, respectively, are both consistent to the structure of the algebra $\mathcal{U}(\mathcal{A})$, i.e. both algebra maps 
(algebra homomorphisms) 7 With these properties, we can compute the actions of any operators on any fields in a consistent manner. Mathematically, all these features assures that our lattice superalgebra actually forms a bialgebra.

Notice that our bialgebra is a mixture of both algebra-like elements, like $Q_{A}^{\text {lat }}$ or $P_{\mu}^{\text {lat }}$, and group-like elements, like $T_{b}$. The latter have their inverse, $T_{b}^{-1}$. The former would also have a sort of inverse, $-Q_{A}^{\text {lat }}$ and $-P_{\mu}^{\text {lat }}$, implying the naïve connection between group and algebra. In fact, we need one more ingredient, namely an antipode, to claim that the DKKN lattice superalgebra is a Hopf algebra, and it is essentially a map to give the "inverse" element for each operator. It would be introduced as a linear map such that satisfies the identity

$$
\cdot \circ(S \otimes \mathrm{id}) \circ \Delta=\cdot \circ(\mathrm{id} \otimes S) \circ \Delta=\eta \circ \epsilon,
$$

where we have used the notation $\cdot(a \otimes b)=a \cdot b$ for the product of operators. We define it explicitly, on the single operators, as

$$
\begin{gathered}
S\left(Q_{A}^{\mathrm{lat}}\right)=-T_{a_{A}^{1}}^{-1} \cdot(-1)^{\mathcal{F}} \cdot Q_{A}^{\mathrm{lat}} \cdot T_{a_{A}^{\mathrm{r}}}^{-1}, \quad S\left(P_{\mu}^{\mathrm{lat}}\right)=-T_{a^{1} \hat{\mu}}^{-1} \cdot P_{\mu}^{\mathrm{lat}} \cdot T_{a^{\mathrm{r}} \hat{\mu}}^{-1}, \\
S\left(T_{b}\right)=T_{b}^{-1}, \quad S\left((-1)^{\mathcal{F}}\right)=(-1)^{-\mathcal{F}}=(-1)^{\mathcal{F}}
\end{gathered}
$$

and extend it so that it becomes linear and anti-algebraic in the sense $S(a \cdot b)=S(b) \cdot S(a), S(\mathbb{1})=$ $\mathbb{1},(a, b \in \mathcal{U}(\mathcal{A}))$. In fact it is shown that the anti-algebraic nature automatically follows if the identity (3.38) holds for the antipode. Here we just see what this identity implies in our superalgebra, without digging into the detail. Applying the first two terms of (3.38) on $Q_{A}^{\text {lat }}$, we find 8

$$
\begin{aligned}
\cdot \circ(S \otimes \mathrm{id}) \circ \Delta\left(Q_{A}^{\mathrm{lat}}\right) & =\cdot \circ(S \otimes \mathrm{id})\left(Q_{A}^{\mathrm{lat}} \otimes T_{a_{A}^{\mathrm{r}}}+(-1)^{\mathcal{F}} \cdot T_{a_{A}^{1}} \otimes Q_{A}^{\text {lat }}\right) \\
& =\cdot\left(-T_{a_{A}^{1}}^{-1} \cdot(-1)^{\mathcal{F}} \cdot Q_{A}^{\mathrm{lat}} \cdot T_{a_{A}^{\mathrm{r}}}^{-1} \otimes T_{a_{A}^{\mathrm{r}}}+T_{a_{A}^{1}}^{-1} \cdot(-1)^{\mathcal{F}} \otimes Q_{A}^{\mathrm{lat}}\right) \\
& =-T_{a_{A}^{1}}^{-1} \cdot(-1)^{\mathcal{F}} \cdot Q_{A}^{\text {lat }}+T_{a_{A}^{1}}^{-1} \cdot(-1)^{\mathcal{F}} \cdot Q_{A}^{\text {lat }}=0,
\end{aligned}
$$

and

$$
\begin{aligned}
\cdot \circ(\mathrm{id} \otimes S) \circ \Delta\left(Q_{A}^{\mathrm{lat}}\right) & =\cdot \circ(\mathrm{id} \otimes S)\left(Q_{A}^{\mathrm{lat}} \otimes T_{a_{A}^{\mathrm{r}}}+(-1)^{\mathcal{F}} \cdot T_{a_{A}^{1}} \otimes Q_{A}^{\mathrm{lat}}\right) \\
& =\cdot\left(Q_{A}^{\mathrm{lat}} \otimes T_{a_{A}^{\mathrm{r}}}^{-1}-(-1)^{\mathcal{F}} \cdot T_{a_{A}^{\mathrm{l}}} \otimes T_{a_{A}^{\mathrm{l}}}^{-1} \cdot(-1)^{\mathcal{F}} \cdot Q_{A}^{\mathrm{lat}} \cdot T_{a_{A}^{\mathrm{r}}}^{-1}\right) \\
& =Q_{A}^{\mathrm{lat}} \cdot T_{a_{A}^{\mathrm{r}}}^{-1}-Q_{A}^{\mathrm{lat}} \cdot T_{a_{A}^{\mathrm{r}}}^{-1}=0,
\end{aligned}
$$

while the last terms gives

$$
\eta \circ \epsilon\left(Q_{A}^{\text {lat }}\right)=0 .
$$

Thus the identity (3.38) holds for the operator $Q_{A}^{\text {lat }}$ with the definition (3.39). Similar calculations show that $P_{\mu}^{\text {lat }}$ also obeys the identity. As for $T_{b}$, we compute

$$
\cdot \circ(S \otimes \mathrm{id}) \circ \Delta\left(T_{b}\right)=\cdot \circ(S \otimes \mathrm{id})\left(T_{b} \otimes T_{b}\right)=\cdot\left(T_{b}^{-1} \otimes T_{b}\right)=\mathbb{1},
$$

and

$$
\cdot \circ(\mathrm{id} \otimes S) \circ \Delta\left(T_{b}\right)=\cdot \circ(\mathrm{id} \otimes S)\left(T_{b} \otimes T_{b}\right)=\cdot\left(T_{b} \otimes T_{b}^{-1}\right)=\mathbb{1}
$$

whereas

$$
\eta \circ \epsilon\left(T_{b}\right)=\mathbb{1},
$$

\footnotetext{
${ }^{7}$ These conditions are the same as imposing the product $m$ and unit $\eta$ should be coalgebra maps.

${ }^{8}$ We use here $S\left((-1)^{\mathcal{F}} \cdot T_{b}\right)=S\left(T_{b}\right) \cdot S\left((-1)^{\mathcal{F}}\right)$ as explicitly shown as (3.49).
} 
again showing the consistency. Let us calculate the antipodes of products of operators with the use of the identity (3.38). Applying the l.h.s. of the identity on $T_{b} \cdot T_{c}$,

$$
\begin{aligned}
\cdot \circ(S \otimes \mathrm{id}) \circ \Delta\left(T_{b} \cdot T_{c}\right) & =\cdot \circ(S \otimes \mathrm{id})\left(T_{b} \cdot T_{c} \otimes T_{b} \cdot T_{c}\right)=\cdot\left(S\left(T_{b} \cdot T_{c}\right) \otimes T_{b} \cdot T_{c}\right) \\
& =S\left(T_{b} \cdot T_{c}\right) \cdot\left(T_{b} \cdot T_{c}\right),
\end{aligned}
$$

and the r.h.s.

$$
\eta \circ \epsilon\left(T_{b} \cdot T_{c}\right)=\eta\left(\epsilon\left(T_{b}\right) \epsilon\left(T_{c}\right)\right)=\eta(1)=\mathbb{1},
$$

so that the identity reads

$$
S\left(T_{b} \cdot T_{c}\right)=\left(T_{b} \cdot T_{c}\right)^{-1}=T_{c}^{-1} \cdot T_{b}^{-1}=S\left(T_{c}\right) \cdot S\left(T_{b}\right),
$$

showing the anti-algebraic nature of the antipode. Just in a similar manner can we show generally

$$
S\left(g_{1} \cdots g_{n}\right)=S\left(g_{n}\right) \cdots S\left(g_{1}\right), \quad g_{i}=T_{b} \quad \text { or } \quad(-1)^{\mathcal{F}} .
$$

Applying next on $T_{b} \cdot Q_{A}^{\text {lat }}$, the 1.h.s. is

$$
\begin{aligned}
\cdot \circ(S \otimes \mathrm{id}) \circ \Delta\left(T_{b} \cdot Q_{A}^{\mathrm{lat}}\right) & =\cdot \circ(S \otimes \mathrm{id})\left(T_{b} \cdot Q_{A}^{\mathrm{lat}} \otimes T_{b} \cdot T_{a_{A}^{\mathrm{r}}}+T_{b} \cdot(-1)^{\mathcal{F}} \cdot T_{a_{A}^{1}} \otimes T_{b} \cdot Q_{A}^{\text {lat }}\right) \\
& =S\left(T_{b} \cdot Q_{A}^{\mathrm{lat}}\right) \cdot T_{b} \cdot T_{a_{A}^{\mathrm{r}}}+S\left(T_{b} \cdot(-1)^{\mathcal{F}} \cdot T_{a_{A}^{1}}\right) \cdot T_{b} \cdot Q_{A}^{\text {lat }},
\end{aligned}
$$

while the r.h.s. is

$$
\eta \circ \epsilon\left(T_{b} \cdot Q_{A}^{\mathrm{lat}}\right)=\eta\left(\epsilon\left(T_{b}\right) \epsilon\left(Q_{A}^{\mathrm{lat}}\right)\right)=\eta(0)=0,
$$

thus the identity gives

$S\left(T_{b} \cdot Q_{A}^{\mathrm{lat}}\right)=-S\left(T_{b} \cdot(-1)^{\mathcal{F}} \cdot T_{a_{A}^{1}}\right) \cdot T_{b} \cdot Q_{A}^{\mathrm{lat}} \cdot T_{a_{a}^{\mathrm{r}}}^{-1} \cdot T_{b}^{-1}=-T_{a_{A}^{1}}^{-1} \cdot(-1)^{\mathcal{F}} \cdot Q_{A}^{\mathrm{lat}} \cdot T_{a_{a}^{\mathrm{r}}}^{-1} \cdot T_{b}^{-1}=S\left(Q_{A}^{\mathrm{lat}}\right) \cdot S\left(T_{b}\right)$.

Now we proceed to the calculation for $Q_{A}^{\text {lat }} \cdot Q_{B}^{\text {lat }}$ : the l.h.s. reads

$$
\begin{aligned}
& \cdot \circ(S \otimes \mathrm{id}) \circ \Delta\left(Q_{A}^{\text {lat }} \cdot Q_{B}^{\text {lat }}\right) \\
& =\cdot \circ(S \otimes \mathrm{id})\left(Q_{A}^{\mathrm{lat}} \cdot Q_{B}^{\mathrm{lat}} \otimes T_{a_{A}^{\mathrm{r}}} \cdot T_{a_{B}^{\mathrm{r}}}+Q_{A}^{\mathrm{lat}} \cdot(-1)^{\mathcal{F}} \cdot T_{a_{B}^{1}} \otimes T_{a_{A}^{\mathrm{r}}} \cdot Q_{B}^{\mathrm{lat}}\right. \\
& \left.+(-1)^{\mathcal{F}} \cdot T_{a_{A}^{1}} \cdot Q_{B}^{\text {lat }} \otimes Q_{A}^{\text {lat }} \cdot T_{a_{B}^{\mathrm{r}}}+(-1)^{\mathcal{F}} \cdot T_{a_{A}^{1}} \cdot(-1)^{\mathcal{F}} \cdot T_{a_{B}^{1}} \otimes Q_{A}^{\text {lat }} \cdot Q_{B}^{\text {lat }}\right) \\
& =S\left(Q_{A}^{\mathrm{lat}} \cdot Q_{B}^{\mathrm{lat}}\right) \cdot T_{a_{A}^{\mathrm{r}}} \cdot T_{a_{B}^{\mathrm{r}}}+S\left(Q_{A}^{\mathrm{lat}} \cdot(-1)^{\mathcal{F}} \cdot T_{a_{B}^{\mathrm{l}}}\right) \cdot T_{a_{A}^{\mathrm{r}}} \cdot Q_{B}^{\mathrm{lat}} \\
& +S\left((-1)^{\mathcal{F}} \cdot T_{a_{A}^{1}} \cdot Q_{B}^{\mathrm{lat}}\right) \cdot Q_{A}^{\mathrm{lat}} \cdot T_{a_{B}^{\mathrm{r}}}+S\left((-1)^{\mathcal{F}} \cdot T_{a_{A}^{1}} \cdot(-1)^{\mathcal{F}} \cdot T_{a_{B}^{1}}\right) \cdot Q_{A}^{\mathrm{lat}} \cdot Q_{B}^{\mathrm{lat}},
\end{aligned}
$$

and the r.h.s.

$$
\eta \circ \epsilon\left(Q_{A}^{\text {lat }} \cdot Q_{B}^{\text {lat }}\right)=\eta \circ\left(\epsilon\left(Q_{A}^{\text {lat }}\right) \epsilon\left(Q_{B}^{\text {lat }}\right)\right)=\eta(0)=0,
$$

so that the identity requires that

$$
\begin{aligned}
S\left(Q_{A}^{\mathrm{lat}} \cdot Q_{B}^{\mathrm{lat}}\right) \cdot T_{a_{A}^{\mathrm{r}}} T_{a_{B}^{\mathrm{r}}} & =T_{a_{B}^{1}}^{-1} \cdot(-1)^{\mathcal{F}} \cdot T_{a_{A}^{1}}^{-1} \cdot(-1)^{\mathcal{F}} \cdot Q_{A}^{\mathrm{lat}} \cdot T_{a_{A}^{\mathrm{r}}}^{-1} \cdot T_{a_{A}^{\mathrm{r}}} \cdot Q_{B}^{\mathrm{lat}} \\
& +T_{a_{B}^{\mathrm{l}}}^{-1} \cdot(-1)^{\mathcal{F}} \cdot Q_{B}^{\mathrm{lat}} \cdot T_{a_{B}^{\mathrm{r}}}^{-1} \cdot T_{a_{A}^{\mathrm{l}}}^{-1} \cdot(-1)^{\mathcal{F}} \cdot Q_{A}^{\mathrm{lat}} \cdot T_{a_{B}^{\mathrm{r}}} \\
& -T_{a_{B}^{\mathrm{l}}}^{-1} \cdot(-1)^{\mathcal{F}} \cdot T_{a_{A}^{\mathrm{l}}}^{-1} \cdot(-1)^{\mathcal{F}} \cdot Q_{A}^{\mathrm{lat}} \cdot Q_{B}^{\mathrm{lat}},
\end{aligned}
$$

i.e. $\quad S\left(Q_{A}^{\mathrm{lat}} \cdot Q_{B}^{\mathrm{lat}}\right)=T_{a_{B}^{\mathrm{l}}}^{-1} \cdot(-1)^{\mathcal{F}} \cdot Q_{B}^{\mathrm{lat}} \cdot T_{a_{B}^{\mathrm{r}}}^{-1} \cdot T_{a_{A}^{\mathrm{l}}}^{-1} \cdot(-1)^{\mathcal{F}} \cdot Q_{A}^{\mathrm{lat}} \cdot T_{a_{A}^{\mathrm{r}}}^{-1}=S\left(Q_{B}^{\mathrm{lat}}\right) \cdot S\left(Q_{A}^{\mathrm{lat}}\right)$,

thus we find that the anti-algebraic nature of the antipode map holds regardless of the fermionic nature of the supercharges. The other identity $\cdot \circ(\mathrm{id} \otimes S)=\eta \circ \epsilon$ also gives the same consequences. 
With all these algebraic consistencies satisfied, we conclude that the universal enveloping algebra, $\mathcal{U}(\mathcal{A})$, i.e. our lattice superalgebra, can be regarded as a Hopf algebra. We now move on to the construction of a field theory which has this Hopf algebraic symmetry. We follow the general scheme formulated by Oeckl [41] as braided quantum field theory (BQFT). To this purpose, we have to specify the complete algebraic nature of the fields which is consistent to the algebraic nature of the Hopf algebra. This requires one more nontrivial ingredient called braiding, or "shifted commutation" in our language. It is in a way a generalization of the statistics of fields. In short, the covariance of the field theory under the Hopf algebraic symmetry force us to introduce the braiding in a consistent way. We will see this in what follows for our specific case of the lattice superalgebra and the corresponding field theory.

\subsection{Shift Structure as a Braiding}

Here we explain why we need the braiding or shift structure in the space of fields, beginning with a simple illustration. Suppose we are considering a normal supersymmetry with a bosonic field $\phi$ and a fermionic field $\psi$. Needless to say, bosonic fields commute with any other fields, while fermionic fields anticommute only with other fermions. Now take a supertransformation $Q \phi=\chi$ with a normal supercharge $Q$ which is supposed to obey the Leibniz rule $Q\left(\varphi_{1} \varphi_{2}\right)=$ $Q \varphi_{1} \varphi_{2}+(-1)^{\left|\varphi_{1}\right|} \varphi_{1} Q \varphi_{2}$. In the Hopf algebraic description we may say that it has the coproduct $\Delta(Q)=Q \otimes \mathbb{1}+(-1)^{\mathcal{F}} \mathbb{1} \otimes Q$ as before. We "know" that the field $\chi$ is fermionic, as a field of supertransformation of a boson $\phi$. The point is that this fact is indeed inevitable; we are forced to chose $\chi$ to be fermionic for the algebraic consistency. In fact, note that the quantity $Q(\phi \psi)=\chi \psi+\phi(Q \psi)$ is equal to $Q(\psi \phi)=(Q \psi) \phi-\psi \chi$, because $\phi$ is defined as a boson, i.e. $\phi \psi=\psi \phi$. Comparing these two relations, we find $\chi \psi+\psi \chi=(Q \psi) \phi-\phi(Q \psi)$, which is zero again due to that $\phi$ is bosonic. This results in that $\chi \psi=-\psi \chi$, "proving" that $\chi$ is a fermion. The essence for this proof is twofold: the one is the coproduct structure of the transformation operator $Q$, especially the factor $(-1)^{\mathcal{F}}$, and the other is the covariance of exchanging fields under the transformation $Q$, namely, when we exchange the order of a product of fields and then apply the transformation with $Q$, the result is the same as the quantity obtained by first applying the transformation with $Q$ on the product and then exchanging the order of the transformed object. The property of fields under exchanging the order in a product is nothing but the statistics of the fields. Here we have just seen a natural and obvious fact that the statistics of fields should be consistent to the algebraic structure and covariance of transformations which apply on the fields. It might be still worth stressing it, however, because it is the reason we need the braiding for our present application of the Hopf algebraic symmetry on fields. It is also the reason of that we think of the braiding as giving a generalized statistics. We are going to investigate these issues in detail in the following.

Let us introduce the general notion of exchanging the order of fields. We denote the exchanged object of $\varphi_{1} \otimes \varphi_{2}$ as

$$
\Psi_{X_{1}, X_{2}}\left(\varphi_{1} \otimes \varphi_{2}\right), \quad \varphi_{1} \in X_{1}, \varphi_{2} \in X_{2} .
$$

The map $\Psi$ is called a braiding when it satisfies some natural consistency conditions (see appendix A). The trivial braiding is given as the normal transposition, and, in the application to the link formalism, we assume that the scalar fields on sites of the lattice would have the trivial braiding nature;

$$
\Psi_{X_{\mathrm{s}}, X_{\mathrm{s}}}\left(\phi_{1} \otimes \phi_{2}\right)=\phi_{2} \otimes \phi_{1}, \quad \phi_{1}, \phi_{2} \in X_{\mathrm{s}}: \text { scalar fields on sites. }
$$

Repeating the argument above, we may apply $Q_{A}^{\text {lat }}$ on the product of scalar fields, or equivalently, take the action of coproduct of $Q_{A}^{\text {lat }}$ as

$$
\Delta\left(Q_{A}^{\mathrm{lat}}\right) \triangleright\left(\phi_{1} \otimes \phi_{2}\right)=\left(Q_{A}^{\mathrm{lat}} \phi_{1}\right) \otimes \phi_{2}\left(x+a_{A}^{\mathrm{r}}\right)+\phi_{1}\left(x+a_{A}^{\mathrm{l}}\right) \otimes\left(Q_{A}^{\mathrm{lat}} \phi_{2}\right) .
$$


Similarly on the exchanged product,

$$
\begin{aligned}
\Delta\left(Q_{A}^{\text {lat }}\right) \triangleright\left(\phi_{2} \otimes \phi_{1}\right) & =\Delta\left(Q_{A}^{\text {lat }}\right) \triangleright \Psi_{X_{\mathrm{s}}, X_{\mathrm{s}}}\left(\phi_{1} \otimes \phi_{2}\right) \\
& =\left(Q_{A}^{\text {lat }} \phi_{2}\right) \otimes \phi_{1}\left(x+a_{A}^{\mathrm{r}}\right)+\phi_{2}\left(x+a_{A}^{\mathrm{l}}\right) \otimes\left(Q_{A}^{\text {lat }} \phi_{1}\right) .
\end{aligned}
$$

We now assume the covariance of the braiding under symmetry transformations, or, in other words, we assume that the braiding to be an intertwiner of the transformations. In the present case, this requires that

$$
\Delta\left(Q_{A}^{\mathrm{lat}}\right) \triangleright \Psi_{X_{\mathrm{s}}, X_{\mathrm{s}}}\left(\phi_{1} \otimes \phi_{2}\right)=\Psi^{\prime}\left(\Delta\left(Q_{A}^{\mathrm{lat}}\right) \triangleright\left(\phi_{1} \otimes \phi_{2}\right)\right) .
$$

The l.h.s. is given by (3.59), while the r.h.s. is

$$
\begin{aligned}
\Psi^{\prime}\left(\Delta\left(Q_{A}^{\mathrm{lat}}\right) \triangleright\left(\phi_{1} \otimes \phi_{2}\right)\right)= & \Psi_{X_{\mathrm{f} A}, X_{\mathrm{s}}}\left(\left(Q_{A}^{\mathrm{lat}} \phi_{1}\right) \otimes \phi_{2}\left(x+a_{A}^{\mathrm{r}}\right)\right) \\
& +\Psi_{X_{\mathrm{s}}, X_{\mathrm{f} A}}\left(\phi_{1}\left(x+a_{A}^{\mathrm{l}}\right) \otimes\left(Q_{A}^{\mathrm{lat}} \phi_{2}\right)\right)
\end{aligned}
$$

where we have denoted the space of fermionic fields of the index $A$ as $X_{\mathrm{f} A}$ to which the transformed fields $Q_{A}^{\text {lat }} \phi_{1,2}$ are to belong. Comparing these two equations, and noting that the fields $\phi_{1}$ and $\phi_{2}$ could be completely independent, we find the consequence, with a simple identification, should be

$$
\begin{aligned}
& \Psi_{X_{\mathrm{f} A}, X_{\mathrm{s}}}\left(\left(Q_{A}^{\mathrm{lat}} \phi_{1}\right) \otimes \phi_{2}\left(x+a_{A}^{\mathrm{r}}\right)\right)=\phi_{2}\left(x+a_{A}^{\mathrm{l}}\right) \otimes\left(Q_{A}^{\mathrm{lat}} \phi_{1}\right), \\
& \Psi_{X_{\mathrm{s}}, X_{\mathrm{f} A}}\left(\phi_{1}\left(x+a_{A}^{\mathrm{l}}\right) \otimes\left(Q_{A}^{\mathrm{lat}} \phi_{2}\right)\right)=\left(Q_{A}^{\mathrm{lat}} \phi_{2}\right) \otimes \phi_{1}\left(x+a_{A}^{\mathrm{r}}\right) .
\end{aligned}
$$

These are not the trivial braiding as in (3.57). Instead, these braiding mean that when we exchange the order of the fermion $Q_{A}^{\text {lat }} \phi$ with the other field, it changes the argument of the other field by the amount $a_{A}^{\mathrm{l}}-a_{A}^{\mathrm{r}}$ under the exchange from the left to the right, and by the opposite amount under the exchange from the right to the left. Recalling that the scalar fields obey the trivial braiding, we might interpret this fact as that the transformed fields, fermions, inherited the nontrivial braiding nature from the supercharge, which, in a way, shows the nontrivial braiding already in the structure of the coproduct. In fact, this kind of nontrivial braiding is referred to as the shifted commutation structure in the link formalism.

We have to emphasize here that the "claimed inconsistency" [42] explained in section 2 no longer appears with incorporating this nontrivial braiding in the non-gauged link formalism. Our approach which is purely based on the Hopf algebraic description clarifies the necessity of the braiding and shows how that problem criticized can be resolved.

To confirm how the things work, let us compute another example:

$$
\Delta\left(Q_{B}^{\mathrm{lat}}\right) \triangleright\left(\psi_{1 A}(x) \otimes \phi_{2}(x)\right)=\left(Q_{B}^{\mathrm{lat}} \psi_{1 A}\right)(x) \otimes \phi_{2}\left(x+a_{B}^{\mathrm{r}}\right)-\psi_{1 A}\left(x+a_{B}^{\mathrm{l}}\right) \otimes \psi_{2 B}(x),
$$

where $\psi_{2 B}:=Q_{B}^{\text {lat }} \phi_{2}$, and thus

$$
\begin{aligned}
\Psi^{\prime}\left(\Delta\left(Q_{B}^{\mathrm{lat}}\right) \triangleright\left(\psi_{1 A}(x) \otimes \phi_{2}(x)\right)\right)= & \Psi_{X_{A B}, X_{\mathrm{s}}}\left(\left(Q_{B}^{\mathrm{lat}} \psi_{1 A}\right)(x) \otimes \phi_{2}\left(x+a_{B}^{\mathrm{r}}\right)\right) \\
& -\Psi_{X_{\mathrm{f} A}, X_{\mathrm{f} B}}\left(\psi_{1 A}\left(x+a_{B}^{\mathrm{l}}\right) \otimes \psi_{2 B}(x)\right)
\end{aligned}
$$

whereas

$$
\begin{aligned}
\Delta\left(Q_{B}^{\mathrm{lat}}\right) \triangleright\left(\phi_{2}\left(x+a_{A}^{\mathrm{l}}-a_{A}^{\mathrm{r}}\right) \otimes \psi_{1 A}(x)\right)= & \Delta\left(Q_{B}^{\mathrm{lat}}\right) \triangleright \Psi_{X_{\mathrm{f} A}, X_{\mathrm{s}}}\left(\psi_{1 A}(x) \otimes \phi_{2}(x)\right) \\
= & \psi_{2 B}\left(x+a_{A}^{\mathrm{l}}-a_{A}^{\mathrm{r}}\right) \otimes \psi_{1 A}\left(x+a_{B}^{\mathrm{r}}\right) \\
& +\phi_{2}\left(x+a_{A}^{\mathrm{l}}-a_{A}^{\mathrm{r}}+a_{B}^{\mathrm{l}}\right) \otimes\left(Q_{B}^{\mathrm{lat}} \psi_{1 A}(x)\right) .
\end{aligned}
$$


Here $X_{A B}$ is such that $Q_{B}^{\text {lat }} \psi_{A} \in X_{A B}$. Assuming again the covariance

$$
\Psi^{\prime}\left(\Delta\left(Q_{B}^{\mathrm{lat}}\right) \triangleright\left(\psi_{1 A}(x) \otimes \phi_{2}(x)\right)\right)=\Delta\left(Q_{B}^{\mathrm{lat}}\right) \triangleright \Psi_{X_{\mathrm{f} A}, X_{\mathrm{s}}}\left(\psi_{1 A}(x) \otimes \phi_{2}(x)\right),
$$

we obtain the following braiding relations:

$$
\begin{gathered}
\Psi_{X_{A B}, X_{\mathrm{s}}}\left(\left(Q_{B}^{\mathrm{lat}} \psi_{1 A}\right)(x) \otimes \phi_{2}\left(x+a_{B}^{\mathrm{r}}\right)\right)=\phi_{2}\left(x+a_{A}^{\mathrm{l}}-a_{A}^{\mathrm{r}}+a_{B}^{\mathrm{l}}\right) \otimes\left(Q_{B}^{\mathrm{lat}} \psi_{1 A}(x)\right), \\
\Psi_{X_{\mathrm{f} A}, X_{\mathrm{f} B}}\left(\psi_{1 A}\left(x+a_{B}^{\mathrm{l}}\right) \otimes \psi_{2 B}(x)\right)=-\psi_{2 B}\left(x+a_{A}^{\mathrm{l}}-a_{A}^{\mathrm{r}}\right) \otimes \psi_{1 A}\left(x+a_{B}^{\mathrm{r}}\right) .
\end{gathered}
$$

Notice, in passing, from the first equation of (3.67), we have

$$
\begin{aligned}
& \Psi_{X_{A B}, X_{\mathrm{s}}}\left(\left(Q_{B}^{\mathrm{lat}} \psi_{1 A}\right)(x) \otimes \phi_{2}(x)\right)=\phi_{2}\left(x+a_{A}^{\mathrm{l}}-a_{A}^{\mathrm{r}}+a_{B}^{\mathrm{l}}-a_{B}^{\mathrm{r}}\right) \otimes\left(Q_{B}^{\mathrm{lat}} \psi_{1 A}\right)(x), \\
& \Psi_{X_{A B}, X_{\mathrm{s}}}\left(\left(Q_{A}^{\mathrm{lat}} \psi_{1 B}\right)(x) \otimes \phi_{2}(x)\right)=\phi_{2}\left(x+a_{B}^{\mathrm{l}}-a_{B}^{\mathrm{r}}+a_{A}^{\mathrm{l}}-a_{A}^{\mathrm{r}}\right) \otimes\left(Q_{A}^{\text {lat }} \psi_{1 B}\right)(x),
\end{aligned}
$$

so that, summing up these two,

$$
\begin{aligned}
\Psi_{X_{A B}, X_{\mathrm{s}}} & \left(\left(\left\{Q_{B}^{\mathrm{lat}}, Q_{A}^{\mathrm{lat}}\right\} \phi_{1}\right)(x) \otimes \phi_{2}(x)\right)=2 \tau_{A B}^{\mu} \Psi_{X_{A B}, X_{\mathrm{s}}}\left(\left(P_{\mu}^{\text {lat }} \phi_{1}\right)(x) \otimes \phi_{2}(x)\right) \\
& =\phi_{2}\left(x+a_{A}^{\mathrm{l}}-a_{A}^{\mathrm{r}}+a_{B}^{\mathrm{l}}-a_{B}^{\mathrm{r}}\right) \otimes\left(\left\{Q_{B}^{\text {lat }}, Q_{A}^{\text {lat }}\right\} \phi_{1}\right)(x) \\
& =2 \tau_{A B}^{\mu} \phi_{2}\left(x+a_{A}^{\mathrm{l}}-a_{A}^{\mathrm{r}}+a_{B}^{\mathrm{l}}-a_{B}^{\mathrm{r}}\right) \otimes\left(P_{\mu}^{\text {lat }} \phi_{1}\right)(x) .
\end{aligned}
$$

These examples show that the general braiding relation would be similarly derived. As a rule of sum, we can write it as

$$
\begin{aligned}
& \Psi\left(\varphi_{A_{0} \cdots A_{p}}(x) \otimes \varphi_{B_{0} \cdots B_{q}}^{\prime}(y)\right) \\
& =(-1)^{p q} \varphi_{B_{0} \cdots B_{q}}^{\prime}\left(y+\sum_{i=1}^{p}\left(a_{A_{i}}^{\mathrm{l}}-a_{A_{i}}^{\mathrm{r}}\right)\right) \otimes \varphi_{A_{0} \cdots A_{p}}\left(x-\sum_{i=1}^{q}\left(a_{B_{i}}^{\mathrm{l}}-a_{B_{i}}^{\mathrm{r}}\right)\right),
\end{aligned}
$$

where we have used the abbreviation $\varphi_{A_{0} \cdots A_{p}}:=Q_{A_{p}}^{\text {lat }} \cdots Q_{A_{1}}^{\text {lat }} \varphi_{A_{0}}$, which could just vanish, where $\varphi_{A_{0}}:=\phi$. If we had introduced a scalar field which itself has nontrivial braiding/shift structure, this relation would have even been generalized.

The exchanging of a product of more than three fields should be naturally introduced. In the case of the trivial braiding,

$$
\begin{aligned}
\Psi_{X_{1} \otimes X_{2}, X_{3}}\left(\left(\phi_{1} \otimes \phi_{2}\right) \otimes \phi_{3}\right) & =\phi_{3} \otimes\left(\phi_{1} \otimes \phi_{2}\right)=\phi_{3} \otimes \phi_{1} \otimes \phi_{2}=\Psi_{X_{1}, X_{3}}\left(\left(\phi_{1} \otimes \phi_{3}\right) \otimes \phi_{2}\right) \\
& \left.=\Psi_{X_{1}, X_{3}} \circ \Psi_{X_{2}, X_{3}}\left(\left(\phi_{1} \otimes \phi_{2}\right) \otimes \phi_{3}\right)\right),
\end{aligned}
$$

so that in the general case we extend it to

$$
\Psi_{X_{1} \otimes X_{2}, X_{3}}=\Psi_{X_{1}, X_{3}} \circ \Psi_{X_{2}, X_{3}}, \quad \Psi_{X_{1}, X_{2} \otimes X_{3}}=\Psi_{X_{1}, X_{3}} \circ \Psi_{X_{1}, X_{2}} .
$$

For example,

$$
\Psi_{X_{1}, X_{2} \otimes X_{3}}\left(\phi_{1}(x) \otimes \phi_{2}(x) \otimes \psi_{3 A}(x)\right)=\phi_{2}(x) \otimes \psi_{3 A}(x) \otimes \phi_{1}\left(x-a_{A}^{\mathrm{l}}+a_{A}^{\mathrm{r}}\right) .
$$

For the exchanging with the trivial or constant fields, we should impose

$$
\Psi_{X_{\mathrm{e}}^{0}, X}=\Psi_{X, X_{\mathrm{e}}^{0}}=\mathrm{id}
$$

where $X_{\mathrm{e}}^{0}$ denotes the space of trivial bosonic fields. Using these rules, let us calculate one more example:

$$
\begin{aligned}
(\mathrm{id} \otimes \Delta) \circ \Delta\left(Q_{A}^{\mathrm{lat}}\right) \triangleright\left(\phi_{1} \otimes \phi_{2} \otimes \psi_{3 B}\right)= & \psi_{1 A}(x) \otimes \phi_{2}\left(x+a_{A}^{\mathrm{r}}\right) \psi_{3 B}\left(x+a_{A}^{\mathrm{r}}\right) \\
& +\phi_{1}\left(x+a_{A}^{\mathrm{l}}\right) \otimes \psi_{2 A}(x) \otimes \psi_{3 B}\left(x+a_{A}^{\mathrm{r}}\right) \\
& +\phi_{1}\left(x+a_{A}^{\mathrm{l}}\right) \otimes \phi_{2}\left(x+a_{A}^{\mathrm{l}}\right) \otimes\left(Q_{A}^{\mathrm{lat}} \psi_{3 B}\right)(x),
\end{aligned}
$$


where we have used the coassociativity (3.18). Applying $\Psi_{X_{1}, X_{2} \otimes X_{3}}$, and comparing it with

$$
\begin{aligned}
& (\mathrm{id} \otimes \Delta) \circ \Delta\left(Q_{A}^{\mathrm{lat}}\right) \triangleright\left(\Psi_{X_{1}, X_{2} \otimes X_{3}}\left(\phi_{1}(x) \otimes \phi_{2}(x) \otimes \psi_{3 B}(x)\right)\right) \\
& =(\mathrm{id} \otimes \Delta) \circ \Delta\left(Q_{A}^{\mathrm{lat}}\right) \triangleright\left(\phi_{2}(x) \otimes \psi_{3 B}(x) \otimes \phi_{1}\left(x-a_{B}^{\mathrm{l}}+a_{B}^{\mathrm{r}}\right)\right) \\
& =\psi_{2 A}(x) \otimes \psi_{3 B}\left(x+a_{A}^{\mathrm{r}}\right) \otimes \phi_{1}\left(x+a_{A}^{\mathrm{r}}-a_{B}^{\mathrm{l}}+a_{B}^{\mathrm{r}}\right) \\
& \quad+\phi_{2}\left(x+a_{A}^{\mathrm{l}}\right) \otimes\left(Q_{A}^{\mathrm{lat}} \psi_{3 B}\right)(x) \otimes \phi_{1}\left(x+a_{A}^{\mathrm{r}}-a_{B}^{\mathrm{l}}+a_{B}^{\mathrm{r}}\right) \\
& \quad-\phi_{2}\left(x+a_{A}^{\mathrm{l}}\right) \otimes \psi_{3 B}\left(x+a_{A}^{\mathrm{l}}\right) \otimes \psi_{1 A}\left(x-a_{B}^{\mathrm{l}}+a_{B}^{\mathrm{r}}\right),
\end{aligned}
$$

where we have used (3.73) and (3.18) again, we find that

$$
\begin{aligned}
& \Psi_{X_{1}, X_{2} \otimes X_{3}}\left(\psi_{1 A}(x) \otimes\left(\phi_{2}\left(x+a_{A}^{\mathrm{r}}\right) \otimes \psi_{3 B}\left(x+a_{A}^{\mathrm{r}}\right)\right)\right) \\
& =-\phi_{2}\left(x+a_{A}^{\mathrm{l}}\right) \otimes \psi_{3 B}\left(x+a_{A}^{\mathrm{l}}\right) \otimes \psi_{1 A}\left(x-a_{B}^{\mathrm{l}}+a_{B}^{\mathrm{r}}\right), \\
& \Psi_{X_{1}, X_{2} \otimes X_{3}}\left(\phi_{1}\left(x+a_{A}^{\mathrm{l}}\right) \otimes\left(\psi_{2 A}(x) \otimes \psi_{3 B}\left(x+a_{A}^{\mathrm{r}}\right)\right)\right) \\
& =\psi_{2 A}(x) \otimes \psi_{3 B}\left(x+a_{A}^{\mathrm{r}}\right) \otimes \phi_{1}\left(x+a_{A}^{\mathrm{r}}-a_{B}^{\mathrm{l}}+a_{B}^{\mathrm{r}}\right), \\
& \Psi_{X_{1}, X_{2} \otimes X_{3}}\left(\phi_{1}\left(x+a_{A}^{\mathrm{l}}\right) \otimes\left(\phi_{2}\left(x+a_{A}^{\mathrm{l}}\right) \otimes\left(Q_{A}^{\mathrm{lat}} \psi_{3 B}\right)(x)\right)\right) \\
& =\phi_{2}\left(x+a_{A}^{\mathrm{l}}\right) \otimes\left(Q_{A}^{\mathrm{lat}} \psi_{3 B}\right)(x) \otimes \phi_{1}\left(x+a_{A}^{\mathrm{r}}-a_{B}^{\mathrm{l}}+a_{B}^{\mathrm{r}}\right) .
\end{aligned}
$$

These examples show that the braiding, i.e. the amount of shifts of the arguments of fields induced under exchanging, is additive; for a field $\varphi_{1}$ with the shift $a_{1}$ and another $\varphi_{2}$ with the shift $a_{2}$, the product $\varphi_{1} \otimes \varphi_{2}$ has the shift $a_{1}+a_{2}$. This is a simple consequence of the natural braiding rule (3.72).

These observations motivate us to introduce the notion of shift structure of fields as a kind of an additive "grading" determined with how many supercharges are acting on the scalar fields. We may thus introduce, in addition to the normal graded structure of fields, i.e. bosonic and fermionic statistics, the graded structure which we call the shift structure so that the space of elementary fields $X$ is decomposed in general as

$$
X=\bigoplus_{\text {grading }} X_{\mathrm{e}} \oplus X_{\mathrm{o}}
$$

The space of whole fields, $\hat{X}$, is also decomposed with respect to the shift/grading structure the same way;

$$
\hat{X}=\bigoplus_{n=0}^{\infty} \bigoplus_{\text {grading }} X^{n}
$$

The field contents and their shift structure are determined in each model, mainly with the use of the Leibniz rule consistency conditions. We have to emphasize that this grading structure is especially crucial to define the explicit form of the "momentum" operator $P_{\mu}^{\text {lat }}$. As mentioned at the beginning of the previous subsection, we might have started with taking a difference operator as its representation: $\left(P_{\mu}^{\mathrm{lat}} \phi\right)(x)=a^{-1}\left(\phi\left(x+a^{\mathrm{l}} \hat{\mu}\right)-\phi\left(x+a^{\mathrm{r}} \hat{\mu}\right)\right)$. This, however, doesn't satisfy the relation (3.69), since we have assumed that $\phi$ obeys a trivial braiding and thus $a^{-1}\left(\phi\left(x+a^{1} \hat{\mu}\right)-\phi\left(x+a^{\mathrm{r}} \hat{\mu}\right)\right)$ has the same trivial braiding. We thus need an expression like $\left(P_{\mu}^{\text {lat }} \phi\right)(x)=a^{-1}\left(\phi^{\prime}\left(x+a^{\mathrm{l}} \hat{\mu}\right)-\phi^{\prime}\left(x+a^{\mathrm{r}} \hat{\mu}\right)\right)$ for which $\phi^{\prime}$ has an additional grading to satisfy the relation (3.69). To give a consistent representation for them is important for the formulation and will be treated elsewhere. Here our claim is that the algebraic description presented here can still formalize a field theory with the Hopf algebraic symmetry even if we don't have the 
explicit representation for these graded fields and their "momentum" operators, as is seen in what follows.

Let us note also that our braiding satisfies that

$$
\Psi_{X_{1}, X_{2}} \circ \Psi_{X_{2}, X_{1}}=\mathrm{id}
$$

or equivalently,

$$
\Psi_{X_{2}, X_{1}}=\Psi_{X_{1}, X_{2}}^{-1}
$$

In a standard mathematical terminology this kind of exchanging map $\Psi$ isn't referred to as a braiding, or one may distinguish it from the strictly braided case. Here we use the term braiding in a broader sense, allowing a type of simple nature (3.80). We emphasize that it is still nontrivial in the sense that $\Psi \neq \tau$, where $\tau$ is the simple transposition: $\tau\left(\varphi_{1} \otimes \varphi_{2}\right)=\varphi_{2} \otimes \varphi_{1}$. In fact, our braiding is a transposition plus some shifts of the arguments of fields up to the statistics factors. This should be compared with the statistics of usual bosons and fermions; for that case the braid is nothing but the simple exchanging up to the statistics. We could therefore describe these facts as that the fields which represent our Hopf algebraic lattice superalgebra naturally obtain a braiding structure which expresses slightly more generalized statistics than the usual one 9

According to the general discussion (see appendix $[\mathrm{A}$ ), it seems that the simple braiding structure (3.80) might be given as an explicit formula (A.20) when the corresponding Hopf algebra is triangular. We find that this is indeed the case at least formally; our symmetry algebra could be identified as a triangular Hopf algebra with an additional grading structure, and the braiding (3.70) be given with the corresponding (quasi-)triangular structure $\mathcal{R}$. To see this, let us first introduce a formal expression for the shift operator $T_{b}$

$$
T_{b}=\exp \left(b^{\mu} \partial_{\mu}\right)
$$

We write this as if the continuum derivative operator $\partial_{\mu}$ were introduced on the lattice; however it must be understood as a formal operator and only well-defined when exponentiated to give the lattice proper operator $T_{b}$. We may impose

$$
\Delta\left(\partial_{\mu}\right)=\partial_{\mu} \otimes \mathbb{1}+\mathbb{1} \otimes \partial_{\mu}, \quad \epsilon\left(\partial_{\mu}\right)=0, \quad S\left(\partial_{\mu}\right)=-\partial_{\mu},
$$

which should be interpreted as formal equivalents of the relations (3.8) and (3.38) for $T_{b}$. We then recall that the generator $Q_{A}^{\text {lat }}$ has a kind of grading as an amount of the shift $a_{A}:=a_{A}^{\mathrm{l}}-a_{A}^{\mathrm{r}}$ induced under exchanging $Q_{A}^{\mathrm{lat}} \varphi$ with other fields. We may express this fact with introducing another operator $L^{\mu}$ such that

$$
a\left[L^{\mu}, Q_{A}^{\mathrm{lat}}\right]=\left(a_{A}\right)^{\mu} Q_{A}^{\mathrm{lat}}, \quad \text { i.e. } \quad\left[L^{\mu}, Q_{A}^{\mathrm{lat}}\right]=l_{A}^{\mu} Q_{A}^{\mathrm{lat}},
$$

where $l_{A}^{\mu}=a^{-1}\left(a_{A}\right)^{\mu}$. Since $P_{\mu}^{\text {lat }}$ is given as $P_{\mu}^{\text {lat }} \sim\left\{Q_{A}^{\text {lat }}, Q_{B}^{\text {lat }}\right\}$, it also has the grading as in

$$
a\left[L^{\mu}, P_{\nu}^{\text {lat }}\right]=a_{P}(\hat{\nu})^{\mu} P_{\nu}^{\text {lat }}=a_{P} \delta_{\nu}^{\mu} P_{\nu}^{\text {lat }}, \quad \text { i.e. } \quad\left[L^{\mu}, P_{\nu}^{\text {lat }}\right]=l_{P} \delta_{\nu}^{\mu} P_{\nu}^{\text {lat }},
$$

where $a_{P}:=a^{1}-a^{\mathrm{r}}$ and $l_{P}:=a^{-1} a_{P}$. We list the other relations

$$
\left[L^{\mu}, T_{b}\right]=\left[L^{\mu},(-1)^{\mathcal{F}}\right]=\left[L^{\mu}, L^{\nu}\right]=0,
$$

where the first two are due to the fact that neither $T_{b}$ nor $(-1)^{\mathcal{F}}$ induces shift and the latter one is automatic because of the "Abelian" nature of (3.84), (3.85) and the others. For completeness, we set

$$
\Delta\left(L^{\mu}\right)=L^{\mu} \otimes \mathbb{1}+\mathbb{1} \otimes L^{\mu}, \quad \epsilon\left(L^{\mu}\right)=0, \quad S\left(L^{\mu}\right)=-L^{\mu} .
$$

\footnotetext{
${ }^{9} \mathrm{~A}$ well-known example of generalized statistics is that of anyons, for which the exchanging map is strictly braided in general. Our statistics is thus more like the usual statistics than the anyonic one.
} 
Now let

$$
\mathcal{R}:=\exp \left(a L^{\mu} \otimes \partial_{\mu}-a \partial_{\mu} \otimes L^{\mu}+i \pi \mathcal{F} \otimes \mathcal{F}\right) .
$$

We can show that this formal operator $\mathcal{R} \in \mathcal{U}(\mathcal{A}) \otimes \mathcal{U}(\mathcal{A})$ is invertible and satisfies the relations

$$
\begin{gathered}
\tau \circ \Delta(h)=\mathcal{R} \cdot \Delta(h) \cdot \mathcal{R}^{-1}, \\
(\Delta \otimes \mathrm{id}) \mathcal{R}=\mathcal{R}_{13} \mathcal{R}_{23}, \quad(\operatorname{id} \otimes \Delta) \mathcal{R}=\mathcal{R}_{13} \mathcal{R}_{12} .
\end{gathered}
$$

(See appendix $\mathrm{A}$ for the notation.) Notice first that $\mathcal{R}^{-1}$ is given as

$$
\mathcal{R}^{-1}=\exp \left(-a L^{\mu} \otimes \partial_{\mu}+a \partial_{\mu} \otimes L^{\mu}+i \pi \mathcal{F} \otimes \mathcal{F}\right)
$$

(recall that $\mathcal{F}$ only gives integer numbers), and so that

$$
\mathcal{R}_{21}=\exp \left(a \partial_{\mu} \otimes L^{\mu}-a L^{\mu} \otimes \partial_{\mu}+i \pi \mathcal{F} \otimes \mathcal{F}\right)=\mathcal{R}^{-1} .
$$

As for the first relation in (3.89), compute

$$
\mathcal{R} \cdot \Delta(h) \cdot \mathcal{R}^{-1}=\sum_{n=0}^{\infty} \frac{1}{n !}(\operatorname{ad}(L))^{n} \Delta(h),
$$

where we have written $L:=a L^{\mu} \otimes \partial_{\mu}-a \partial_{\mu} \otimes L^{\mu}+i \pi \mathcal{F} \otimes \mathcal{F}$ just for simplicity, and used ad to denote the Lie derivative. For $h=Q_{A}^{\text {lat }}$,

$$
\begin{aligned}
\operatorname{ad}(L) \Delta\left(Q_{A}^{\mathrm{lat}}\right)=\left[a L^{\mu} \otimes \partial_{\mu}-a \partial_{\mu} \otimes L^{\mu}+i \pi \mathcal{F} \otimes \mathcal{F}, Q_{A}^{\mathrm{lat}} \otimes T_{a_{A}^{\mathrm{r}}}+(-1)^{\mathcal{F}} \cdot T_{a_{A}^{1}} \otimes Q_{A}^{\text {lat }}\right] \\
=\left[a L^{\mu} \otimes \partial_{\mu}+i \pi \mathcal{F} \otimes \mathcal{F}, Q_{A}^{\mathrm{lat}} \otimes T_{a_{A}^{\mathrm{r}}}\right]+\left[-a \partial_{\mu} \otimes L^{\mu}+i \pi \mathcal{F} \otimes \mathcal{F},(-1)^{\mathcal{F}} \cdot T_{a_{A}^{\mathrm{l}}} \otimes Q_{A}^{\text {lat }}\right] \\
=a\left[L^{\mu}, Q_{A}^{\mathrm{lat}}\right] \otimes \partial_{\mu} \cdot T_{a_{A}^{\mathrm{r}}}+i \pi\left[\mathcal{F}, Q_{A}^{\mathrm{lat}}\right] \otimes \mathcal{F} \cdot T_{a_{A}^{\mathrm{r}}} \\
\quad-a \partial_{\mu} \cdot(-1)^{\mathcal{F}} \cdot T_{a_{A}^{\mathrm{l}}} \otimes\left[L^{\mu}, Q_{A}^{\mathrm{lat}}\right]+i \pi \mathcal{F} \cdot(-1)^{\mathcal{F}} \cdot T_{a_{A}^{1}} \otimes\left[\mathcal{F}, Q_{A}^{\text {lat }}\right] \\
=Q_{A}^{\text {lat }} \otimes\left(\left(a_{A}\right)^{\mu} \partial_{\mu}+i \pi \mathcal{F}\right) \cdot T_{a_{A}^{\mathrm{r}}}+\left(-\left(a_{A}\right)^{\mu} \partial_{\mu}+i \pi \mathcal{F}\right) \cdot(-1)^{\mathcal{F}} T_{a_{A}^{1}} \otimes Q_{A}^{\text {lat }},
\end{aligned}
$$

so that

$$
(\operatorname{ad}(L))^{n} \Delta\left(Q_{A}^{\mathrm{lat}}\right)=Q_{A}^{\mathrm{lat}} \otimes\left(\left(a_{A}\right)^{\mu} \partial_{\mu}+i \pi \mathcal{F}\right)^{n} \cdot T_{a_{A}^{\mathrm{r}}}+\left(-\left(a_{A}\right)^{\mu} \partial_{\mu}+i \pi \mathcal{F}\right)^{n} \cdot(-1)^{\mathcal{F}} \cdot T_{a_{A}^{1}} \otimes Q_{A}^{\mathrm{lat}} .
$$

We therefore obtain

$$
\begin{aligned}
& \mathcal{R} \cdot \Delta\left(Q_{A}^{\text {lat }}\right) \cdot \mathcal{R}^{-1} \\
& =Q_{A}^{\text {lat }} \otimes \exp \left(\left(a_{A}\right)^{\mu} \partial_{\mu}+i \pi \mathcal{F}\right) \cdot T_{a_{A}^{\mathrm{r}}}+\exp \left(-\left(a_{A}\right)^{\mu} \partial_{\mu}+i \pi \mathcal{F}\right) \cdot(-1)^{\mathcal{F}} \cdot T_{a_{A}^{1}} \otimes Q_{A}^{\text {lat }} \\
& =Q_{A}^{\text {lat }} \otimes(-1)^{\mathcal{F}} \cdot T_{a_{A}^{1}}+T_{a_{A}^{\mathrm{r}}} \otimes Q_{A}^{\text {lat }}=\tau \circ \Delta\left(Q_{A}^{\text {lat }}\right),
\end{aligned}
$$

since

$$
\exp \left( \pm\left(a_{A}\right)^{\mu} \partial_{\mu}+i \pi \mathcal{F}\right)=\exp \left( \pm\left(a_{A}^{\mathrm{l}}\right)^{\mu} \partial_{\mu}\right) \cdot \exp \left(\mp\left(a_{A}^{\mathrm{r}}\right)^{\mu} \partial_{\mu}\right) \cdot \exp (i \pi \mathcal{F})=T_{a_{A}^{\mathrm{l}}}^{ \pm} \cdot T_{a_{A}^{\mathrm{r}}}^{\mp} \cdot(-1)^{\mathcal{F}}
$$

A simpler calculation leads to similar result for $h=P_{\mu}^{\text {lat }}$ too. For $h=T_{b},(-1)^{\mathcal{F}}, L^{\mu}$, it is rather clear that

$$
\mathcal{R} \cdot \Delta(h) \cdot \mathcal{R}^{-1}=\Delta(h)=\tau \circ \Delta(h) .
$$


Thus the first equation in (3.89) indeed holds for the choice (3.88) of $\mathcal{R}$. The second relation follows as

$$
\begin{aligned}
&(\Delta \otimes \mathrm{id}) \mathcal{R}= \exp \left(a \Delta\left(L^{\mu}\right) \otimes \partial_{\mu}-a \Delta\left(\partial_{\mu}\right) \otimes L^{\mu}+i \pi \Delta(\mathcal{F}) \otimes \mathcal{F}\right) \\
&= \exp \left(a L^{\mu} \otimes \mathbb{1} \otimes \partial_{\mu}-a \partial_{\mu} \otimes \mathbb{1} \otimes L^{\mu}+i \pi \mathcal{F} \otimes \mathbb{1} \otimes \mathcal{F}\right. \\
&\left.\quad+a \mathbb{1} \otimes L^{\mu} \otimes \partial_{\mu}-a \mathbb{1} \otimes \partial_{\mu} \otimes L^{\mu}+i \pi \mathbb{1} \otimes \mathcal{F} \otimes \mathcal{F}\right) \\
&=\exp \left(a L^{\mu} \otimes \mathbb{1} \otimes \partial_{\mu}-a \partial_{\mu} \otimes \mathbb{1} \otimes L^{\mu}+i \pi \mathcal{F} \otimes \mathbb{1} \otimes \mathcal{F}\right) \\
& \cdot \exp \left(a \mathbb{1} \otimes L^{\mu} \otimes \partial_{\mu}-a \mathbb{1} \otimes \partial_{\mu} \otimes L^{\mu}+i \pi \mathbb{1} \otimes \mathcal{F} \otimes \mathcal{F}\right)=\mathcal{R}_{13} \cdot \mathcal{R}_{23} .
\end{aligned}
$$

The third one is almost the same.

We have thus shown that the formal operator $\mathcal{R}$ given as (3.88) is a quasitriangular structure and, due to (3.91), our lattice superalgebra is identified as a triangular Hopf algebra. The whole spaces of fields, as representation spaces of a triangular Hopf algebra, would be braided by $\mathcal{R}$ as in

$$
\Psi=\tau \circ \mathcal{R} \triangleright,
$$

which agrees with our formula (3.70) as now seen. We need the representation of $L^{\mu}$ on the elementary fields. First for normal scalar fields $\{\phi, \cdots\}$ let

$$
L^{\mu} \triangleright \phi=0 \cdot \phi=0 .
$$

For the other fields in the irreducible supermultiplet to which the above bosonic fields belong, the actions of $L^{\mu}$ are automatically determined by the algebra (3.84). For instance, on $\psi_{A}:=Q_{A}^{\text {lat }} \phi$, we find

$$
L^{\mu} \triangleright \psi_{A}=L^{\mu} \triangleright\left(Q_{A}^{\mathrm{lat}} \phi\right)=\left(\left[L^{\mu}, Q_{A}^{\mathrm{lat}}\right]+Q_{A}^{\mathrm{lat}} \cdot L^{\mu}\right) \triangleright \phi=l_{A}^{\mu} Q_{A}^{\mathrm{lat}} \phi=l_{A}^{\mu} \psi_{A} .
$$

Then inductively, we find for $\varphi_{A_{1} \cdots A_{n}}=Q_{A_{n}}^{\text {lat }} \cdots Q_{A_{1}}^{\text {lat }} \phi$ that

$$
L^{\mu} \triangleright \varphi_{A_{1} \cdots A_{n}}=\left(l_{A_{1}}+\cdots+l_{A_{n}}\right)^{\mu} \varphi_{A_{1} \cdots A_{n}} .
$$

These relations express explicitly the grading structure of fields explained above. We thus compute

$$
\begin{aligned}
& \mathcal{R} \triangleright\left(\varphi_{A_{1} \cdots A_{p}}(x) \otimes \varphi_{B_{1} \cdots B_{q}}(y)\right) \\
& =\exp \left(\mathbb{1} \otimes\left(\left(a_{A_{1}}+\cdots+a_{A_{p}}\right)^{\mu} \partial_{\mu}\right)-\left(\left(a_{B_{1}}+\cdots+a_{B_{q}}\right)^{\mu} \partial_{\mu}\right) \otimes \mathbb{1}+i \pi p q \mathbb{1} \otimes \mathbb{1}\right) \\
& \quad \triangleright\left(\varphi_{A_{1} \cdots A_{p}}(x) \otimes \varphi_{B_{1} \cdots B_{q}}(y)\right) \\
& =(-1)^{p q}\left(\mathbb{1} \otimes T_{a_{A_{1}}+\cdots+a_{A_{p}}}\right) \cdot\left(T_{a_{B_{1}}+\cdots+a_{B_{q}}}^{-1} \otimes \mathbb{1}\right) \triangleright\left(\varphi_{A_{1} \cdots A_{p}}(x) \otimes \varphi_{B_{1} \cdots B_{q}}(y)\right) \\
& =\varphi_{A_{1} \cdots A_{p}}\left(x-\sum_{i=1}^{p} a_{B_{i}}\right) \otimes \varphi_{B_{1} \cdots B_{q}}\left(y+\sum_{i=1}^{q} a_{A_{i}}\right) .
\end{aligned}
$$

Since here $a_{A_{i}}=a_{A_{i}}^{\mathrm{l}}-a_{A_{i}}^{\mathrm{r}}$ etc., we have shown that the equation (3.99) does reproduce the general braiding rule (3.70).

It is worth pointing out that our quasitriangular structure $\mathcal{R}$ can be written as

$$
\mathcal{R}=\chi_{21} \cdot \mathcal{R}_{0} \cdot \chi^{-1}, \quad \mathcal{R}_{0}:=\exp (i \pi \mathcal{F} \otimes \mathcal{F}),
$$

with some invertible operator $\chi \in \mathcal{U}(\mathcal{A}) \otimes \mathcal{U}(\mathcal{A})$ which satisfies so-called the 2-cocycle condition

$$
(\chi \otimes \mathbb{1}) \cdot(\Delta \otimes \mathrm{id}) \chi=(\mathbb{1} \otimes \chi) \cdot(\mathrm{id} \otimes \Delta) \chi,
$$


and the counital condition

$$
(\epsilon \otimes \mathrm{id}) \chi=(\mathrm{id} \otimes \epsilon) \chi=\mathbb{1} .
$$

Such an operator is not necessarily unique. We take one specific example to illustrate it:

$$
\begin{gathered}
\chi:=\exp \left(a \partial_{\mu} \otimes L^{1 \mu}+a L^{\mathrm{r} \mu} \otimes \partial_{\mu}\right), \\
\chi_{21}=\exp \left(a L^{1 \mu} \otimes \partial_{\mu}+a \partial_{\mu} \otimes L^{\mathrm{r} \mu}\right), \quad \chi^{-1}=\exp \left(-a \partial_{\mu} \otimes L^{1 \mu}-a L^{\mathrm{r} \mu} \otimes \partial_{\mu}\right),
\end{gathered}
$$

where we have introduced two more operators $L^{1 \mu}$ and $L^{\mathrm{r} \mu}$ such that $L^{\mu}=L^{1 \mu}-L^{\mathrm{r} \mu}$, namely,

$$
a\left[L^{\mathrm{l}, \mathrm{r} \mu}, Q_{A}^{\mathrm{lat}}\right]=\left(a_{A}^{\mathrm{l}, \mathrm{r}}\right)^{\mu} Q_{A}^{\mathrm{lat}}, \quad \text { etc. },
$$

with coproduct, counit and antipode formulae similar to those of $L^{\mu}$. It is easy to see that (3.104) actually holds for this operator $\chi$. The cocycle condition is fulfilled as

$$
\begin{aligned}
&\text { (l.h.s. })= \exp \left(a \partial_{\mu} \otimes L^{\mathrm{l} \mu} \otimes \mathbb{1}+a L^{\mathrm{r} \mu} \otimes \partial_{\mu} \otimes \mathbb{1}\right) \cdot \exp \left(a \Delta\left(\partial_{\mu}\right) \otimes L^{\mathrm{l} \mu}+a \Delta\left(L^{\mathrm{r} \mu}\right) \otimes \partial_{\mu}\right) \\
&= \exp \left(a \partial_{\mu} \otimes L^{1 \mu} \otimes \mathbb{1}+a L^{\mathrm{r} \mu} \otimes \partial_{\mu} \otimes \mathbb{1}\right. \\
&\left.\quad+a \partial_{\mu} \otimes \mathbb{1} \otimes L^{1 \mu}+a \mathbb{1} \otimes \partial_{\mu} \otimes L^{1 \mu}+a L^{\mathrm{r} \mu} \otimes \mathbb{1} \otimes \partial_{\mu}+a \mathbb{1} \otimes L^{\mathrm{r} \mu} \otimes \partial_{\mu}\right) \\
&= \exp \left(\mathbb{1} \otimes a \partial_{\mu} \otimes L^{\mathrm{l} \mu}+\mathbb{1} \otimes a L^{\mathrm{r} \mu} \otimes \partial_{\mu}\right. \\
&\left.\quad \quad+a \partial_{\mu} \otimes L^{1 \mu} \otimes \mathbb{1}+a \partial_{\mu} \otimes \mathbb{1} \otimes L^{\mathrm{l} \mu}+a L^{\mathrm{r} \mu} \otimes \partial_{\mu} \otimes \mathbb{1}+a L^{\mathrm{r} \mu} \otimes \mathbb{1} \otimes \partial_{\mu}\right) \\
& \quad \exp \left(\mathbb{1} \otimes a \partial_{\mu} \otimes L^{1 \mu}+\mathbb{1} \otimes a L^{\mathrm{r} \mu} \otimes \partial_{\mu}\right) \cdot \exp \left(a \partial_{\mu} \otimes \Delta\left(L^{1 \mu}\right)+a L^{\mathrm{r} \mu} \otimes \Delta\left(\partial_{\mu}\right)\right) \\
&=(\text { r.h.s. }),
\end{aligned}
$$

while the counitality is clear because $\epsilon\left(\partial_{\mu}\right)=\epsilon\left(L^{1, \mathrm{r} \mu}\right)=0$. We thus conclude from these results that our lattice superalgebra $\mathcal{U}(\mathcal{A})$ with the quasitriangular structure $\mathcal{R}$ could be understood as so-called the twist by the cocycle element $\chi$ of some other Hopf algebra $\mathcal{U}(\mathcal{A})_{0}$ with the simple quasitriangular structure $\mathcal{R}_{0}$. The "untwisted" Hopf algebra $\mathcal{U}(\mathcal{A})_{0}$ has the same algebra and counit as those of $\mathcal{U}(\mathcal{A})$ but its coproduct and antipode are such that

$$
\begin{aligned}
& \Delta(h)=\chi \cdot \Delta_{0}(h) \cdot \chi^{-1}, \\
& S(h)=U \cdot S_{0}(h) \cdot U^{-1}, \quad U:=\cdot(\operatorname{id} \otimes S) \chi, \quad U^{-1}=\cdot(S \otimes \mathrm{id}) \chi^{-1} .
\end{aligned}
$$

Thus for $h=T_{b},(-1)^{\mathcal{F}}, L^{\mu}$, we find $\Delta_{0}(h)=\Delta(h)$, whereas for $h=Q_{A}^{\text {lat }}, P_{\mu}^{\text {lat }}$, we can show that

$$
\Delta_{0}\left(Q_{A}^{\text {lat }}\right)=Q_{A}^{\text {lat }} \otimes \mathbb{1}+(-1)^{\mathcal{F}} \otimes Q_{A}^{\text {lat }}, \quad \Delta_{0}\left(P_{\mu}^{\text {lat }}\right)=P_{\mu}^{\text {lat }} \otimes \mathbb{1}+\mathbb{1} \otimes P_{\mu}^{\text {lat }} .
$$

Since in the present case

$$
U=\exp \left(-a L_{+}^{\mu} \cdot \partial_{\mu}\right), \quad U^{-1}=\exp \left(a L_{+}^{\mu} \cdot \partial_{\mu}\right), \quad L_{+}^{\mu}:=L^{1 \mu}+L^{\mathrm{r} \mu},
$$

antipodes as well remain unchanged for $h=T_{b},(-1)^{\mathcal{F}}, L^{1, \mathrm{r} \mu}$, but changed again for $h=$ $Q_{A}^{\text {lat }}, P_{\mu}^{\text {lat }}$ :

$$
S_{0}\left(Q_{A}^{\text {lat }}\right)=-(-1)^{\mathcal{F}} \cdot Q_{A}^{\text {lat }}, \quad S_{0}\left(P_{\mu}^{\text {lat }}\right)=-P_{\mu}^{\text {lat }},
$$

as seen with the use of

$$
U \cdot Q_{A}^{\mathrm{lat}} \cdot U^{-1}=\exp \left(\left(a_{A}^{\mathrm{l}}+a_{A}^{\mathrm{r}}\right)^{\mu} \partial_{\mu}\right) \cdot Q_{A}^{\mathrm{lat}}=T_{a_{A}^{1}} \cdot T_{a_{A}^{\mathrm{r}}} \cdot Q_{A}^{\mathrm{lat}}
$$

and of similar for $P_{\mu}^{\text {lat }}$. 
We have found that the (un)twisted Hopf algebra $\left(\mathcal{U}(\mathcal{A})_{0}, \mathcal{R}_{0}\right)$ becomes much simpler and has the form of a normal universal enveloping Lie superalgebra of normal supersymmetry. This result might seem confusing because under the twisting the algebraic structure of the original Hopf algebra remains the same and operators themselves don't take any transformations; if such simpler Hopf algebra exits, could we just begin with it without taking the deformed one $(\mathcal{U}(\mathcal{A}), \mathcal{R})$ ? Actually we can equally formulate the whole story with the simpler Hopf algebra $\left(\mathcal{U}(\mathcal{A})_{0}, \mathcal{R}_{0}\right)$, but notice that this twisting transformation is only possible with the nontrivial "charge" or "grading" operators $L^{1, \mathrm{r} \mu}$ at our disposal, and that the twisted Hopf algebra keeps them as well. On our original Hopf algebra $(\mathcal{U}(\mathcal{A}), \mathcal{R})$, these have a natural interpretation as those assigning how fields are geometrically put on the lattice and how operators affect on such a geometrical structure. On the twisted Hopf algebra $\left(\mathcal{U}(\mathcal{A})_{0}, \mathcal{R}_{0}\right)$, this kind of interpretation is less clear since $\Delta_{0}, S_{0}$, etc., just have normal structure and, nevertheless, these operators $L^{1, \mathrm{r} \mu}$ must be included for the whole algebra to be represented exactly. This last observation would be quite crucial, particularly when compared with the no-go theorem presented in [43], since in the twisted algebra the "momentum" operator obeys the exact, not modified, Leibniz rule for which no local and translationally covariant representation is proved to exist. We expect that the nontrivial grading of the "momentum" operator may fill the gap of difficulties.

One more aspect to be mentioned here is that the multiplication rule for the fields representing the algebra should become modified correspondingly under the twisting. Let us denote by $(\hat{X}, m)$ and $\left(\hat{X}_{0}, m_{0}\right)$ the spaces of fields which represent, respectively, the deformed algebra $(\mathcal{U}(\mathcal{A}), \mathcal{R})$ and the twisted algebra $\left(\mathcal{U}(\mathcal{A})_{0}, \mathcal{R}_{0}\right)$. Here $m$ and $m_{0}$ are the multiplication maps on the spaces $\hat{X}$ and $\hat{X}_{0}$, respectively. On products for $\hat{X}, h \in \mathcal{U}(\mathcal{A})$ acts covariantly as we have seen in the previous subsection: $h \triangleright m\left(\varphi \otimes \varphi^{\prime}\right)=m\left(\Delta(h) \triangleright\left(\varphi \otimes \varphi^{\prime}\right)\right)$. According to the theory of twisting (see appendix $\underline{\mathrm{A}}$ ), $h \in \mathcal{U}(\mathcal{A})_{0}$ can act covariantly on products of fields for $\hat{X}_{0}$ only with the product

$$
m_{0}:=m \circ \chi \triangleright
$$

(note that the twisting from $(\mathcal{U}(\mathcal{A}), \mathcal{R})$ to $\left(\mathcal{U}(\mathcal{A})_{0}, \mathcal{R}_{0}\right)$ is given with $\left.\chi^{-1}\right)$, as in

$$
h \triangleright m_{0}\left(\varphi \otimes \varphi^{\prime}\right)=m_{0}\left(\Delta_{0}(h) \triangleright\left(\varphi \otimes \varphi^{\prime}\right)\right) .
$$

Suppose that this product $m_{0}$ is "commutative" in the sense that

$$
m_{0} \circ \Psi_{0}=m_{0}, \quad \text { i.e. } \quad m_{0} \circ \tau \circ \mathcal{R}_{0} \triangleright=m_{0},
$$

which means commutative up to the statistics factor induced by $\mathcal{R}_{0}$. This assumption would be natural because the twisted algebra $\left(\mathcal{U}(\mathcal{A})_{0}, \mathcal{R}_{0}\right)$ has the simple Hopf algebraic structure which is symmetric under exchanging orders of any objects. It turns out that then the multiplication $m$ is again commutative up to the nontrivial statistics $\Psi$ (thus noncommutative in the standard sense):

$$
\begin{aligned}
m \circ \Psi & =m_{0} \circ \chi^{-1} \triangleright \circ \tau \circ \mathcal{R} \triangleright=m_{0} \circ \tau \circ\left(\chi_{21}^{-1} \cdot \mathcal{R} \cdot \chi\right) \triangleright \circ \chi^{-1} \triangleright \\
& =m_{0} \circ \Psi_{0} \circ \chi^{-1} \triangleright=m_{0} \circ \chi^{-1} \triangleright=m .
\end{aligned}
$$

This consequence in a way shows that multiplication rule should incorporate the statistics in the obvious manner so that it becomes commutative up to the statistics. When the statistics is itself nontrivial, this notion of the commutativity up to the statistics may be expressed as just a noncommutativity in the standard sense. In our case, we have

$$
\varphi_{A_{1} \cdots A_{p}}(x) \cdot \varphi_{B_{1} \cdots B_{q}}(y)=(-1)^{p q} \varphi_{B_{1} \cdots B_{q}}\left(y+\sum_{i=1}^{p} a_{A_{i}}\right) \cdot \varphi_{A_{1} \cdots A_{p}}\left(x-\sum_{i=1}^{q} a_{B_{i}}\right) .
$$

We regard it as the consequence of either the lattice-deformed statistics, or the mild noncommutativity, and may use the notation $\varphi * \varphi^{\prime}$ to emphasize its noncommutative nature. 
We finally recall that the space of fields on the lattice $\hat{X}$, defined in (3.1), forms an algebra. It actually forms a Hopf algebra in a natural way [41, 54]:

$$
\begin{array}{ll}
m\left(\varphi_{1} \otimes \varphi_{2}\right)=\varphi_{1} \cdot \varphi_{2} & \text { (product), } \\
\eta(1)=\mathbf{1} & \text { (unit) }, \\
\Delta(\varphi)=\varphi \otimes \mathbf{1}+\mathbf{1} \otimes \varphi, \quad \Delta(\mathbf{1})=\mathbf{1} \otimes \mathbf{1} & \text { (coproduct), } \\
\epsilon(\varphi)=0, \quad \epsilon(\mathbf{1})=1 & \text { (counit) } \\
S(\varphi)=-\varphi, \quad S(\mathbf{1})=\mathbf{1} & \text { (antipode) }
\end{array}
$$

where $\varphi \in X$. This Hopf algebraic structure shouldn't be confused with that of the symmetry operators $\mathcal{U}(\mathcal{A})$ acting on $\hat{X}$. In addition to these Hopf algebraic structure, the space $\hat{X}$ has the braiding/shift structure $\Psi$ which obeys the consistency conditions (3.72) and (3.74). With the use of the braiding, the Hopf algebraic structure is extended to the whole field space $\hat{X}$; coproduct, counit, and antipode of a product of two elementary fields $\varphi_{1}, \varphi_{2} \in X$ are defined by

$$
\begin{aligned}
\Delta\left(\varphi_{1} \cdot \varphi_{2}\right) & :=(m \otimes m) \circ(\mathrm{id} \otimes \Psi \otimes \mathrm{id})\left(\Delta\left(\varphi_{1}\right) \otimes \Delta\left(\varphi_{2}\right)\right), \\
\epsilon\left(\varphi_{1} \cdot \varphi_{2}\right) & :=\epsilon\left(\varphi_{1}\right) \epsilon\left(\varphi_{2}\right), \\
S\left(\varphi_{1} \cdot \varphi_{2}\right) & :=m \circ \Psi\left(S\left(\varphi_{1}\right) \otimes S\left(\varphi_{2}\right)\right),
\end{aligned}
$$

and generalized inductively to any products in $\hat{X}$. One of the most crucial nature for this braiding structure is that it must be covariant under the symmetry operations. In fact we recall that the braiding structure is inevitable only for the covariant consistency under the Hopf algebraic symmetry: $a \triangleright \circ \Psi=\Psi \circ a \triangleright, a \in \mathcal{U}(\mathcal{A})$. With all these properties, the space $\hat{X}$ is called a braided Hopf algebra, or, more precisely, Hopf algebra in a braided category. We thus claim that the link formalism naturally treats the space of fields as a braided Hopf algebra with a Hopf algebraic symmetry, for which the general BQFT formalism can apply. We now see this application in the next subsection.

\subsection{Perturbative Definition of Supersymmetry on the Lattice as a Braided Quantum Field Theory}

Following the general theory of BQFT given in [41, we are now constructing a lattice theory which has the Hopf algebraic symmetry introduced in the previous subsections. Before going to concrete examples, let us here briefly review the general framework. The crucial ingredient to define a quantum field theory is the path integral. For defining a perturbation theory it is enough to introduce it as a formal Gaussian integral, such that the total functional derivative under it is supposed to be zero. We therefore need the functional derivative, which is defined as below.

We now introduce the functional derivative with respect to $\varphi \in X$ as in

$$
\frac{\delta}{\delta \varphi(x)} \varphi(y)=\delta^{D}(x-y) .
$$

Following more abstract definition in [41], we write this as

$$
\mathrm{ev}\left(\frac{\delta}{\delta \varphi(x)} \otimes \varphi(y)\right):=\frac{\delta}{\delta \varphi(x)} \varphi(y),
$$

introducing the evaluation map ev. It is a kind of natural contraction of $X$ and $X^{*}$, where $X^{*}$ is the dual space to $X$ composed of $\delta / \delta \varphi$. Similarly we might introduce the opposite one, a kind of completeness relation, as

$$
\operatorname{coev}(\lambda):=\lambda \sum_{x} \varphi(x) \otimes \frac{\delta}{\delta \varphi(x)}
$$


These maps are characterized with the identities:

$$
(\mathrm{ev} \otimes \mathrm{id})(\mathrm{id} \otimes \mathrm{coev})=\mathrm{id}_{X^{*}}, \quad(\mathrm{id} \otimes \mathrm{ev})(\mathrm{coev} \otimes \mathrm{id})=\mathrm{id}_{X} .
$$

The functional derivative can be naturally extended to the one which acts on the whole space of fields $\hat{X}$ as in the following way. On a product of two elementary fields $\varphi_{1}, \varphi_{2} \in X$, the functional derivative acts with the use of a braided Leibniz rule as in

$$
\frac{\delta}{\delta \varphi(x)}\left(\varphi_{1}\left(x_{1}\right) \cdot \varphi_{2}\left(x_{2}\right)\right)=\frac{\delta}{\delta \varphi(x)} \varphi_{1}\left(x_{1}\right) \cdot \varphi_{2}\left(x_{2}\right)+\cdot\left[\Psi^{-1}\left(\frac{\delta}{\delta \varphi(x)} \otimes \varphi_{1}\left(x_{1}\right)\right)\left(\mathbf{1} \otimes \varphi_{2}\left(x_{2}\right)\right)\right] .
$$

On products of more than three fields are extended inductively. Needless to say, the derivative trivially commutes with a constant field (see (3.74)), and gives zero when it acts on a constant. More rigorous definition of the functional derivative is given in [41, 54].

Now we can introduce a Gaussian integration with the following property:

$$
\int \frac{\delta}{\delta \varphi}\left(\mathcal{O}[\varphi] e^{-S_{0}}\right)=0, \quad \mathcal{O}[\varphi] \in \hat{X}, \quad \frac{\delta}{\delta \varphi} \in X^{*},
$$

where $\exp \left(-S_{0}\right) \in \hat{X}$ is the corresponding Gaussian factor. In the application to the field theory, $S_{0}$ is interpreted as the free part of the action. Notice that this integration is formally understood as the one which satisfies the property (3.127) without referring to its real values. This way of abstract definition is already enough to define a perturbation theory and to compute correlation functions with arbitrary order, since for such computations only the ratio of the integral to another integral, partition function, is needed (this is nothing different from the path integral in a usual field theory), and that ratio can be computed only with these algebraic properties.

We now introduce a kind of propagator. Letting

$$
\frac{\delta}{\delta \varphi(x)} e^{-S_{0}}=-\gamma\left(\frac{\delta}{\delta \varphi(x)}\right) e^{-S_{0}}
$$

we define an object $\gamma: X^{*} \rightarrow X$. More specifically, it is given as

$$
\gamma\left(\frac{\delta}{\delta \varphi(x)}\right)=\frac{\delta}{\delta \varphi(x)} S_{0},
$$

which roughly corresponds to the inverse propagator, so that the propagator is in a way given as $\gamma^{-1}$. This naïve argument can be justified shortly.

The free $n$-point correlation function is now defined by

$$
Z_{n}^{(0)}\left(\alpha_{n}\right):=\frac{\int \alpha_{n} e^{-S_{0}}}{\int e^{-S_{0}}}, \quad \alpha_{n} \in X^{n} .
$$

The superscript (0) stands for the free theory. In this definition, the denominator, denoted here tentatively as $Z^{(0)}$, might be interpreted as the free partition function, but in the general case we don't have any definition to directly compute it as mentioned above. Still this definition is enough to calculate the correlation functions of any order. To see this argument, notice first that

$$
\alpha_{n} \varphi e^{-S_{0}}=\alpha_{n} \gamma\left(\gamma^{-1}(\varphi)\right) e^{-S_{0}}=-\alpha_{n} \gamma^{-1}(\varphi)\left(e^{-S_{0}}\right),
$$

where we have used the definition (3.128) and the fact that $\gamma^{-1}(\varphi) \in X^{*}$ and so is a functional derivative. We then find, using the braided Leibniz rule, that

$$
-\alpha_{n} \gamma^{-1}(\varphi)\left(e^{-S_{0}}\right)=-\left(\gamma^{-1}\left(\varphi^{\alpha_{n}}\right)\left(\alpha_{n}^{\varphi} e^{-S_{0}}\right)-\gamma^{-1}\left(\varphi^{\alpha_{n}}\right)\left(\alpha_{n}^{\varphi}\right) e^{-S_{0}}\right)
$$


where we have denoted the "shifted" field as $\varphi^{\alpha_{n}}$ and $\alpha_{n}^{\varphi}$, with the superscripts implying the amount of shift:10. We thus find

$$
\begin{aligned}
\int \alpha_{n} \varphi e^{-S_{0}} & =-\int\left(\gamma^{-1}\left(\varphi^{\alpha_{n}}\right)\left(\alpha_{n}^{-\varphi} e^{-S_{0}}\right)-\gamma^{-1}\left(\varphi^{\alpha_{n}}\right)\left(\alpha_{n}^{\varphi}\right) e^{-S_{0}}\right) \\
& =\int \gamma^{-1}\left(\varphi^{\alpha_{n}}\right)\left(\alpha_{n}^{\varphi}\right) e^{-S_{0}} .
\end{aligned}
$$

In the second equality, the first term vanishes because its a total derivative under the path integral. We therefore obtain a basic formula

$$
Z^{(0)}\left(\alpha_{n} \varphi\right)=Z^{(0)}\left(\gamma^{-1}\left(\varphi^{\alpha_{n}}\right)\left(\alpha_{n}^{\varphi}\right)\right) .
$$

For example, putting $\alpha=\mathbb{1}(n=0)$ in the formula above (3.134), it is clear that

$$
Z_{1}^{(0)}(\varphi)=0
$$

The simplest nontrivial example is given for $n=1$ by taking $\alpha_{1}=\varphi_{1}\left(x_{1}\right) \in X$ and $\varphi=\varphi_{2}\left(x_{2}\right) \in$ $X$ in (3.134), so that

$$
\begin{aligned}
Z_{2}^{(0)}\left(\varphi_{1}\left(x_{1}\right) \varphi_{2}\left(x_{2}\right)\right) & =Z_{2}^{(0)}\left(\gamma^{-1}\left(\varphi_{2}\left(x_{2}+a_{\varphi_{1}}\right)\right)\left(\varphi_{1}\left(x+a_{\varphi_{2}}\right)\right)\right) \\
& =\gamma^{-1}\left(\varphi_{2}\left(x_{2}+a_{\varphi_{1}}\right)\right)\left(\varphi_{1}\left(x+a_{\varphi_{2}}\right)\right) .
\end{aligned}
$$

The other formulae can be computed inductively using (3.134). The general results are summarized as follows:

$$
\begin{aligned}
Z_{2}^{(0)} & =\mathrm{ev} \circ\left(\gamma^{-1} \otimes \mathrm{id}\right) \circ \Psi, \\
Z_{2 n}^{(0)} & =\left(Z_{2}^{(0)}\right)^{n} \circ[2 n-1]_{\Psi}^{\prime} ! ! \\
Z_{2 n+1}^{(0)} & =0,
\end{aligned}
$$

where

$$
\begin{aligned}
{[2 n-1]_{\Psi}^{\prime} ! ! } & :=\left([1]_{\Psi}^{\prime} \otimes \mathrm{id}^{2 n-1}\right) \circ\left([3]_{\Psi}^{\prime} \otimes \mathrm{id}^{2 n-3}\right) \circ \cdots \circ\left([2 n-1]_{\Psi}^{\prime} \otimes \mathrm{id}\right), \\
{[n]_{\Psi}^{\prime} } & :=\mathrm{id}^{n}+\mathrm{id}^{n-2} \otimes \Psi^{-1}+\cdots+\Psi_{1, n-1}^{-1} .
\end{aligned}
$$

These are the Wick's theorem in the BQFT formalism.

When an interaction is turned on, we can treat the theory perturbatively. Let the action be $S=S_{0}+\lambda S_{\text {int }}$. The $n$-point correlation function now reads

$$
\begin{aligned}
Z_{n}\left(\alpha_{n}\right) & :=\frac{\int \alpha_{n} e^{-S}}{\int e^{-S}} \\
& =\frac{\int \alpha_{n}\left(1-\lambda S_{\mathrm{int}}+\cdots\right) e^{-S_{0}}}{\int\left(1-\lambda S_{\mathrm{int}}+\cdots\right) e^{-S_{0}}}, \quad \alpha_{n} \in X^{n} .
\end{aligned}
$$

Dividing both the numerator and denominator by the "partition function" $Z^{(0)}$, we find

$$
Z_{n}=\frac{Z_{n}^{(0)}-\lambda Z_{n+k}^{(0)} \circ\left(\mathrm{id}^{n} \otimes S_{\mathrm{int}}\right)+\frac{1}{2} \lambda^{2} Z_{n+2 k}^{(0)} \circ\left(\mathrm{id}^{n} \otimes S_{\mathrm{int}} \otimes S_{\mathrm{int}}\right)+\cdots}{1-\lambda Z_{k}^{(0)} \circ S_{\mathrm{int}}+\frac{1}{2} \lambda^{2} Z_{2 k}^{(0)} \circ\left(S_{\mathrm{int}} \otimes S_{\mathrm{int}}\right)+\cdots},
$$

\footnotetext{
${ }^{10}$ This notational simplicity can only apply to our present case for the specific braiding/shift structure. The general expression with general braiding $\Psi$ is given in [41].
} 
where $k$ is the order of the interaction $S_{\text {int }}$, i.e. $S_{\text {int }} \in X^{k}$, and we have put a map $S_{\text {int }}: \mathbb{C} \rightarrow X^{k}$ with the abuse of notation.

Let us give an example to see the formalism above more explicitly. We here consider $\mathcal{N}=$ $(2,2)$ Wess-Zumino model in two dimensions in the Dirac-Kähler twisted basis. Superalgebra is given as before

$$
\left\{Q^{\text {lat }}, Q_{\mu}^{\text {lat }}\right\}=i \partial_{+\mu}, \quad\left\{\tilde{Q}^{\text {lat }}, Q_{\mu}^{\text {lat }}\right\}=-i \epsilon_{\mu \nu} \partial_{-\nu}
$$

Bosonic fields include scalars $\phi, \sigma$ and auxiliary fields $\tilde{\phi}, \tilde{\sigma}$, whereas fermionic fields are $\psi, \tilde{\psi}, \psi_{\mu}$. Supertransformations are given in Appendix B. The action is given as

$$
\begin{aligned}
S=\sum_{x}[ & \left(\partial_{+\mu}^{\mathrm{lat}} \sigma\right)(x-a \hat{\mu}) \cdot\left(\partial_{-\mu}^{\mathrm{lat}} \phi\right)(x)-\tilde{\sigma}\left(x+a_{1}+a_{2}\right) \cdot \tilde{\phi}(x) \\
& -i \psi(x-a) \cdot \partial_{-\mu}^{\mathrm{lat}} \psi_{\mu}(x)-i \epsilon_{\mu \nu} \tilde{\psi}(x-\tilde{a}) \cdot \partial_{+\mu}^{\mathrm{lat}} \psi_{\nu}(x) \\
& \quad-\partial_{\phi} W\left(x+a_{1}+a_{2}\right) \cdot \tilde{\phi}(x)-\partial_{\sigma} V(x+a+\tilde{a}) \cdot \tilde{\sigma}(x) \\
& \left.+\partial_{\sigma}^{2} V(x+a+\tilde{a}) \cdot \psi(x+\tilde{a}) \cdot \tilde{\psi}(x)-i \epsilon^{\mu \nu} \partial_{\phi}^{2} W\left(x+a_{1}+a_{2}\right) \cdot \psi_{\mu}\left(x+a_{\nu}\right) \cdot \psi_{\nu}(x)\right],
\end{aligned}
$$

where $W$ and $V$ are potentials in the twisted basis. The invariance of the action can be unambiguously seen using the modified Leibniz rule taking care of the specific "staggered" configurations of arguments of the fields as well as of the mildly generalized statistics (3.119).

\subsection{Ward-Takahashi Identities}

Here we follow [54]. The invariance of the correlation functions can be written as

$$
Z_{n}(a \triangleright \chi)=\epsilon(a) Z_{n}(\chi), \quad a \in \mathcal{U}(\mathcal{A}), \quad \chi \in X^{n},
$$

which is the Ward-Takahashi identity corresponding to the Hopf algebraic symmetry $\mathcal{U}(\mathcal{A})$. Just as in the usual field theory, the invariance of the correlation functions follows from the invariance of the action. One obvious difference from the usual case is that, with the nontrivial braiding, the symmetry operators must act on the fields in a manner consistent to the braiding structure. In fact it is shown that the identity (3.145) follows when the following four conditions are satisfied [54]:

1. Invariance of the free action:

$$
a \triangleright \gamma^{-1}(\varphi)=\gamma^{-1}(a \triangleright \varphi)
$$

2. Invariance of the interaction:

$$
a \triangleright S_{\mathrm{int}}=\epsilon(a) S_{\mathrm{int}} .
$$

3. Covariance of the braiding:

$$
\Psi\left(a \triangleright\left(X_{1} \otimes X_{2}\right)\right)=a \triangleright \Psi\left(X_{1} \otimes X_{2}\right) .
$$

4. Invariance of the delta function:

$$
\mathrm{ev}\left(a \triangleright\left(X^{*} \otimes X\right)\right)=\epsilon(a) \operatorname{ev}\left(X^{*} \otimes X\right) .
$$

In our current application, the general formula of Ward-Takahashi identity (3.145) naturally gives the correct identities on the lattice. It is important that the general formula (3.145) can be proved unambiguously only using the algebraic relations. 


\subsection{Nonperturbative Definition?}

In this section, we first extracted the essential requirements for the symmetry operators in the link formalism, concluding that this symmetry is Hopf algebraic. Then we utilized the general framework of BQFT formulated in [41, showing that supersymmetric theory on a lattice in the link formalism can be treated with a formal definition of path integral. This path integral approach, however, only gives a perturbative formulation in general, due to the lack of explicit definition of the path integral. As a field theory on a lattice, this situation wouldn't be satisfactory at all, especially for the application to numerical simulations. It is known in some cases one can define a "braided integral" explicitly [56]. We might be able to apply such an approach to the current problem to define a rigorous path integral on the lattice, which, if possible, should give the nonperturbative definition in this formulation based on the Hopf algebraic symmetry. As we have shown in subsection 3.2, it is also crucial to accommodate the explicit representation of grading nature for the lattice momentum operator.

\section{Conclusion and Discussion}

We have shown how the link formalism is treated as a field theory on a lattice with deformed or modified algebraic symmetry. The deformation of the algebra is indeed identified as the one naturally treated in the framework of the Hopf algebra. We showed this argument explicitly, defining the corresponding Hopf algebraic structures of the supersymmetry algebra for the link formalism. The modified Leibniz rule, which is the crucial notion in the original link formalism, was incorporated as the coproduct structure of the Hopf algebra, whose consistency is assured with the other relevant structures of the algebra. The Hopf algebra introduced this way in fact turned out to be a (quasi)triangular Hopf algebra, which has a nontrivial universal $R$-matrix. When represented on the space of fields, this quasitriangular structure inevitably induce a nontrivial statistics, or a noncommutativity, which is the key ingredient for the consistent representation. With these algebraic descriptions, we could identify the link formalism as a representation theory of a quasitriangular Hopf algebra. On the other hand, it is known that there is a general scheme to construct a quantum field theory which has a Hopf algebraic symmetry, called braided quantum field theory. We applied this general formulation to the link formalism. The construction is purely algebraic. In particular, it defines a path integral using only algebraic properties. One can show that it still gives a well-defined perturbative description of the theory, providing full methods for calculating correlation functions in any order. It also gives a concise formulation to derive the possible Ward-Takahashi identities corresponding to the Hopf algebraic symmetry. We therefore realized the link formalism as a quantum field theory which has the quasitriangular Hopf algebraic symmetry at least in the perturbative sense.

From the consistency of the Hopf algebraic structure, it is required that the lattice momentum operator which is proportional to the difference operator should carry a grading compatible with the shifting nature of the difference operator. In this paper we have not given a concrete representation of this grading structure which may be needed to give an explicit nonperturbative definition of this formulation. We leave this issue for the future investigation.

The algebraic inconsistency pointed out in [42], which is connected with the ordering ambiguity of component fields when applying supersymmetry transformation, is solved by the introduction of braiding structure according to the notion of coproduct for the lattice super charges and the momentum operator in Hopf algebra.

It is then important to ask the question how the continuum limit of this formulation is realized. If one can formulate the braided quantum field theory which respects the Hopf algebraic structure as a concrete representation for modified path integral, the twisted lattice supersymmetry will be kept in the continuum limit since the lattice twisted supersymmetry is exactly 
kept. As we have shown the lattice supersymmetry is kept in the perturbative level of braided quantum field theory. It is still nontrivial question how the symmetry is recovered even in the nonperturbative level. In any case we expect that fine tuning is not needed to keep the supersymmetry in the continuum limit if the formulation of deformed supersymmetry algebra is concretely constructed.

In the formulation of orbifold construction of lattice field theories only a subset of lattice super charges in particular the nilpotent scalar super charge which corresponds to the shiftless charge in the link construction is exactly preserved on the lattice [28, 29, 30, 31, 32, 33, 34, 35, 36]. The lattice super algebra in this case is identified as the same as the continuum twisted supersymmetry algebra. It was stressed that the super charges carrying a shift break the lattice super symmetry in the sense of the continuum twisted superalgebra [34, 35]. Our claim in this paper is that these supercharges carrying the shift may break the continuum twisted supersymmetry but preserve exactly the Hopf algebraic supersymmetry. Thus in the link approach all the lattice super charges are claimed to preserve exactly in the framework of Hopf algebraic supersymmetry. The supersymmetry algebra is deformed from the continuum twisted supersymmetry to Hopf algebraic supersymmetry.

We have not considered the gauge extension of deformed supersymmetry in this paper. It was pointed out that there is similar ordering ambiguity for the lattice super Yang-Mills formulation of link approach [42]. We consider that this problem can be solved similar as non-gauge case by identifying the lattice supersymmetry with gauge symmetry of link approach as Hopf algebraic symmetry. There is, however, yet another problem in the gauge extension; the loss of the gauge invariance due to the link nature of the lattice super charges. A possible solution was proposed by introducing covariantly constant super parameters $\eta_{A}[8]$ :

$$
\left\{\nabla_{B}, \eta_{A}\right\}=0
$$

where $\nabla_{B}$ is super covariant derivative. This is highly nontrivial relation in the sense that the fermionic parameter $\eta_{A}$ carrying a shift should carry an internal space-time dependence caused by the super covariant derivative $\nabla_{B}$ to keep the covariant constancy. Here we may consider that fermionic link variables are defined on the links of internal space-time. In other words the space-time distortion of internal space-time may compensate the required dependence of the fermionic parameter. There is a possibility that gravity may play a role in these questions.

It has been pointed out that the breakdown of the Leibniz rule for the lattice difference operator is inevitable under reasonable assumptions for algebraic property on the lattice [43]. Recent renormalization group analyses confirms this statement from different point of view [27]. In order to realize supersymmetry algebra which includes the momentum operator on the lattice it is most natural to introduce the difference operator in the lattice supersymmetry algebra. While the exact supersymmetry of continuum supersymmetry algebra was realized only for the nilpotent super charge which is the scalar part of the twisted supersymmetry algebra but does not include the crucial momentum dependence. We claim that the deformation of the Lie algebraic continuum supersymmetry to Hopf algebraic supersymmetry on the lattice is inevitable to accommodate the difference operator in the algebra.

It is obviously very important to find concrete representation of the Hopf algebraic supersymmetry algebra on the lattice to obtain "modified" path integral definition of the QFT with this particular braiding structure. As we have already shown this type of mild noncommutativity with shifting nature may be well accommodated by a matrix formulation of lattice noncommutativity [40, 57]. This part of concrete proposal with the necessary formulation of graded momentum lattice operator will be given elsewhere. It would be also interesting to compare the formulation of the link approach with other noncommutative approach [58] and non-lattice formulations [59, 60]. 


\section{Acknowledgments}

We are grateful for useful discussions with S. Arianos, T. Asakawa, P. H. Damgaard, A. Feo, H. B. Nielsen, Y. Sasai and N. Sasakura. This work is supported in part by Japanese Ministry of Education, Science, Sports and Culture under the grant No. 18540245 and INFN research fund.

\section{A Brief Summary of Hopf Algebra}

Here we briefly list the axioms of Hopf algebra and some related notions which are used in this article. For rigorous and complete descriptions, see, for example, [62, 63, 61].

\section{A.1 Hopf Algebra}

A Hopf algebra over a field $k(=\mathbb{C}$ or $\mathbb{R})$ is a vector space $H$ over $k$ which has the following properties 1, 2, 3 and 4,

1. $H$ is a unital associative algebra, so that

- it has a $k$-linear multiplication (or product) map11

$$
\cdot H \otimes H \rightarrow H, \quad \cdot\left(h_{1} \otimes h_{2}\right)=h_{1} \cdot h_{2},
$$

which is associative

$$
\cdot \circ(\cdot \otimes \mathrm{id})=\cdot \circ(\mathrm{id} \otimes \cdot), \quad \text { i.e. } \quad\left(h_{1} \cdot h_{2}\right) \cdot h_{3}=h_{1} \cdot\left(h_{2} \cdot h_{3}\right)
$$

- it has unit element $\mathbb{1}$ which satisfies $\mathbb{1} \cdot h=h \cdot \mathbb{1}=h$, whose existence can be formally expressed as the existence of a $k$-linear map

$$
\eta: k \rightarrow H, \quad \eta(\lambda)=\lambda \mathbb{1}, \lambda \in k .
$$

2. $H$ is a coalgebra. Namely,

- it has a $k$-linear map called coproduct:

$$
\Delta: H \rightarrow H \otimes H, \quad \Delta(h)=\sum_{i} h_{i(1)} \otimes h_{i(2)}, \quad h_{i(1)}, h_{i(2)} \in H,
$$

which satisfies the coassociativity 12

$$
(\Delta \otimes \mathrm{id}) \circ \Delta=(\mathrm{id} \otimes \Delta) \circ \Delta, \quad \text { i.e. } \quad h_{(1)(1)} \otimes h_{(1)(2)} \otimes h_{(2)}=h_{(1)} \otimes h_{(2)(1)} \otimes h_{(2)(2)}
$$

- it has also another $k$-linear map called counit

$$
\epsilon: H \rightarrow k,
$$

which obeys the relation

$$
(\epsilon \otimes \mathrm{id}) \circ \Delta=(\mathrm{id} \otimes \epsilon) \circ \Delta=\mathrm{id}, \quad \text { i.e. } \quad \epsilon\left(h_{(1)}\right) h_{(2)}=\epsilon\left(h_{(2)}\right) h_{(1)}=h .
$$

3. These structures of algebra and coalgebra are compatible with each other. Namely,

\footnotetext{
${ }^{11}$ In what follows we take, unless otherwise specified, $h, h_{1}, h_{2}, \cdots$, to be arbitrary elements of $H$.

${ }^{12}$ We use below much simpler abbreviation $\Delta(h)=h_{(1)} \otimes h_{(2)}$ known as the Sweedler's notation.
} 
- the coproduct and the counit are both algebra maps:

$$
\Delta\left(h_{1} \cdot h_{2}\right)=\Delta\left(h_{1}\right) \cdot \Delta\left(h_{2}\right), \quad \epsilon\left(h_{1} \cdot h_{2}\right)=\epsilon\left(h_{1}\right) \epsilon\left(h_{2}\right) .
$$

4. $H$ has one more map called antipode:

- it has a $k$-linear map

$$
S: H \rightarrow H
$$

which obeys the identity

$$
\cdot(S \otimes \mathrm{id}) \circ \Delta=\cdot(\mathrm{id} \otimes S) \circ \Delta=\eta \circ \epsilon, \quad \text { i.e. } \quad S\left(h_{(1)}\right) \cdot h_{(2)}=h_{(1)} \cdot S\left(h_{(2)}\right)=\epsilon(h) \mathbb{1} .
$$

If the $k$-linear space $H$ satisfies these properties 1, 2, 3, but not 4, it is called a bialgebra.

\section{A.2 Quasitriangular Structure}

A Hopf algebra $H$ is said to be quasitriangular if there exists an invertible element $\mathcal{R} \in H \otimes H$ which satisfies

$$
\begin{gathered}
\tau \circ \Delta h=\mathcal{R} \cdot(\Delta h) \cdot \mathcal{R}^{-1}, \\
(\Delta \otimes \mathrm{id}) \mathcal{R}=\mathcal{R}_{13} \mathcal{R}_{23}, \quad(\mathrm{id} \otimes \Delta) \mathcal{R}=\mathcal{R}_{13} \mathcal{R}_{12},
\end{gathered}
$$

where

$$
\begin{gathered}
\mathcal{R}=\sum \mathcal{R}^{(1)} \otimes \mathcal{R}^{(2)}, \\
\mathcal{R}_{12}=\sum \mathcal{R}^{(1)} \otimes \mathcal{R}^{(2)} \otimes \mathbb{1}, \quad \mathcal{R}_{13}=\sum \mathcal{R}^{(1)} \otimes \mathbb{1} \otimes \mathcal{R}^{(2)}, \quad \mathcal{R}_{23}=\sum \mathbb{1} \otimes \mathcal{R}^{(1)} \otimes \mathcal{R}^{(2)},
\end{gathered}
$$

and $\tau: H \otimes H \rightarrow H \otimes H$ is the transposition map

$$
\tau\left(h_{1} \otimes h_{2}\right)=h_{2} \otimes h_{1}, \quad h_{1}, h_{2} \in H .
$$

The element $\mathcal{R}$, if exists, is called the quasitriangular structure or universal $R$-matrix.

If a quasitriangular structure $\mathcal{R}$ of a quasitriangular Hopf algebra $H$ obeys further the following condition, the Hopf algebra is said to be triangular:

$$
\mathcal{R}_{21} \mathcal{R}=\mathbb{1} \otimes \mathbb{1}, \quad \text { i.e. } \quad \mathcal{R}_{21}=\mathcal{R}^{-1}, \quad \text { where } \quad \mathcal{R}_{21}=\sum \mathcal{R}^{(2)} \otimes \mathcal{R}^{(1)} .
$$

\section{A.3 Action on Algebras}

A (left) action of a Hopf algebra $H$ on an associative algebra $X$ is a representation $\rho: H \rightarrow$ $\operatorname{Lin}(X)$, where $\operatorname{Lin}(X)$ is the algebra of linear maps on $X$, which satisfies the covariance in the following sense

$$
\left\{\begin{array}{l}
h \triangleright\left(\varphi \cdot \varphi^{\prime}\right)=m\left(\Delta(h) \triangleright\left(\varphi \otimes \varphi^{\prime}\right)\right), \\
h \triangleright \mathbf{1}=\epsilon(h) \mathbf{1},
\end{array} \quad \varphi, \varphi^{\prime} \in X .\right.
$$

We have here introduced the notation $h \triangleright \varphi:=\rho(h)(\varphi)$, the product $m$ of $X$ with the abbreviation $\varphi \cdot \varphi^{\prime}:=m\left(\varphi \otimes \varphi^{\prime}\right)$, and the unit $1 \in X$. 


\section{A.4 Braiding}

Let us consider a formal collection 13 of representation spaces $(\mathbf{1}, X, Y, Z, \cdots)$ of a Hopf algebra $H$ together with the collection of tensor products of the representation spaces $(\mathbf{1} \otimes X \cong X \otimes \mathbf{1} \cong$ $X, X \otimes Y,(X \otimes Y) \otimes Z \cong X \otimes(Y \otimes Z), \cdots)$ for which $H$ acts with the coproduct structure $((\Delta h) \triangleright(\varphi \otimes \chi), h \in H, \varphi \in X, \chi \in Y)$. If there exists an invertible intertwiner (isomorphism)

$$
\Psi_{X, Y}: X \otimes Y \rightarrow Y \otimes X, \quad \Psi_{X, Y}(\Delta(h) \triangleright(\varphi \otimes \chi))=\Delta(h) \triangleright \Psi_{X, Y}(\varphi \otimes \chi)
$$

with the properties

$$
\Psi_{X \otimes Y, Z}=\Psi_{X, Z} \circ \Psi_{Y, Z}, \quad \Psi_{X, Y \otimes Z}=\Psi_{X, Z} \circ \Psi_{X, Y},
$$

it unambiguously relates the two representations on $X \otimes Y$ and $Y \otimes X$. It should be compatible with any maps which intertwine the representation spaces as in

$$
\Psi_{Z, W} \circ\left(g_{X Z} \otimes g_{Y W}\right)=\left(g_{Y W} \otimes g_{X Z}\right) \circ \Psi_{X, Y}, \quad g_{X Z}: X \rightarrow Z, \quad g_{Y W}: Y \rightarrow W .
$$

We call this isomorphism $\Psi$ a braid. Strictly speaking, a braid should be such that

$$
\Psi \circ \Psi \neq \mathrm{id}, \quad \text { or } \quad \Psi_{X, Y} \neq \Psi_{Y, X}^{-1},
$$

which means there are two distinct ways in relating $X \otimes Y$ to $Y \otimes X$. It gives a nontrivial rule of exchanging factors of a tensor product, and generalizes the statistics of the representation spaces. If, on the other hand, it satisfies $\Psi \circ \Psi=\mathrm{id}$, the isomorphism is more like a simple transposition and said to be symmetric.

When the Hopf algebra $H$ is quasitriangular, we can express the braiding more explicitly using the quasitriangular structure $\mathcal{R}$ of $H$ and the transposition map $\tau$ (A.13) as in

$$
\Psi_{X, X^{\prime}}\left(\varphi \otimes \varphi^{\prime}\right)=\tau \circ \mathcal{R} \triangleright\left(\varphi \otimes \varphi^{\prime}\right), \quad \varphi \in X, \varphi^{\prime} \in X^{\prime} .
$$

This indeed becomes an invertible intertwiner (A.16) and satisfies the conditions (A.17) and (A.18). We find that

$$
\Psi_{X, X^{\prime}} \circ \Psi_{X^{\prime}, X}=\tau \circ \mathcal{R} \triangleright(\tau \circ \mathcal{R} \triangleright)=\tau\left(\left(\mathcal{R} \cdot \mathcal{R}_{21}\right) \triangleright \circ \tau\right) .
$$

Thus the condition (A.19), that is for $\Psi$ to be strictly braided, is equivalent to

$$
\mathcal{R} \cdot \mathcal{R}_{21} \neq \mathbb{1} \otimes \mathbb{1}
$$

namely that the universal $R$-matrix is really quasitriangular. Equivalently, a symmetric isomorphism $\Psi$ corresponds to the triangular structure $\mathcal{R} \cdot \mathcal{R}_{21}=\mathbb{1} \otimes \mathbb{1}$.

\section{A.5 Twist}

Let $H$ be a Hopf algebra. An invertible element $\chi \in H \otimes H$ is called a (2-)cocycle when it satisfies that

$$
(\chi \otimes \mathbb{1})(\Delta \otimes \mathrm{id}) \chi=(\mathbb{1} \otimes \chi)(\mathrm{id} \otimes \Delta) \chi \quad((2-) \text { cocycle condition }) .
$$

A cocycle $\chi$ is said to be counital if 14

$$
(\epsilon \otimes \mathrm{id}) \chi=\mathbb{1} \quad \text { and } \quad(\mathrm{id} \otimes \epsilon) \chi=\mathbb{1} \quad \text { (counital condition). }
$$

For a quasitriangular Hopf algebra $(H, \mathcal{R})$ and a counital 2-cocycle $\chi$, there exists a new Hopf algebra $\left(H_{\chi}, \mathcal{R}_{\chi}\right)$ which has

\footnotetext{
${ }^{13}$ The notion of braiding would be most suitably defined in terms of category theory. Here instead we just give a simple and intuitive description.

${ }^{14}$ Here actually only one of the two conditions is suffice.
} 
- the same algebra and counit as those for $(H, \mathcal{R})$,

- coproduct: $\Delta_{\chi} h=\chi(\Delta h) \chi^{-1}$,

- antipode: $S_{\chi} h=U(S h) U^{-1}$, where $U=\cdot(\operatorname{id} \otimes S) \chi, U^{-1}=\cdot(S \otimes \mathrm{id}) \chi^{-1}$,

- quasitriangular structure: $\mathcal{R}_{\chi}=\chi_{21} \mathcal{R} \chi^{-1}$, where $\chi_{21}=\tau(\chi)$ with $\tau$ given in (A.13).

The process obtaining the Hopf algebra $\left(H_{\chi}, \mathcal{R}_{\chi}\right)$ from the original one $(H, \mathcal{R})$ is called a twist with the element $\chi$ called a twist element. If $(H, \mathcal{R})$ is triangular, so is $\left(H_{\chi}, \mathcal{R}_{\chi}\right)$.

When a Hopf algebra $H$ acts on an associative algebra $X$ covariantly as in (A.15), the twisted Hopf algebra $H_{\chi}$ with a twist element $\chi$ acts covariantly on a new algebra $X_{\chi}$ with a new product

$$
\varphi * \varphi^{\prime}:=m \circ \chi^{-1} \triangleright\left(\varphi \otimes \varphi^{\prime}\right)
$$

and with the same unit. The new product $*$ is associative and in general noncommutative even if the original product $\cdot$ is commutative.

\section{B $\mathcal{N}=(2,2)$ Wess-Zumino Model in Two Dimensions}

We list here the explicit supertransformation formulae for $\mathcal{N}=(2,2)$ Wess-Zumino model in two dimensions. The superalgebra is

$$
\begin{gathered}
\left\{Q^{\text {lat }}, Q_{\mu}^{\text {lat }}\right\}=P_{+\mu}^{\text {lat }}, \quad\left\{\tilde{Q}^{\text {lat }}, Q_{\mu}^{\text {lat }}\right\}=-\epsilon_{\mu \nu} P_{-\nu}^{\text {lat }} \\
\left(P_{ \pm \mu}^{\text {lat }}:=i \partial_{ \pm \mu}\right)
\end{gathered}
$$

with the other commutators just vanishing. The field contents are $\left\{\phi, \sigma, \psi, \psi_{\mu}, \tilde{\psi}, \tilde{\phi}, \tilde{\sigma}\right\}$, for which the supertransformations are as follows:

$$
\begin{aligned}
& Q^{\text {lat }} \phi=0, \quad Q_{\mu}^{\text {lat }} \phi=\psi_{\mu}, \quad \tilde{Q}^{\text {lat }} \phi=0, \\
& Q^{\text {lat }} \psi_{\nu}=i \partial_{+\nu} \phi, \quad Q_{\mu}^{\text {lat }} \psi_{\nu}=-\epsilon_{\mu \nu} \tilde{\phi}, \quad \tilde{Q}^{\text {lat }} \psi_{\nu}=-i \epsilon_{\nu \mu} \partial_{-\mu} \phi, \\
& Q^{\text {lat } \tilde{\phi}}=-i \epsilon_{\mu \nu} \partial_{+\mu} \psi_{\nu}, \quad Q_{\mu}^{\text {lat }} \tilde{\phi}=0, \quad \tilde{Q}^{\text {lat } \tilde{\phi}}=i \partial_{-\mu} \psi_{\mu}, \\
& Q^{\text {lat }} \sigma=-\psi, \quad Q_{\mu}^{\text {lat }} \sigma=0, \quad \tilde{Q}^{\text {lat }} \sigma=-\tilde{\psi}, \\
& Q^{\text {lat }} \psi=0, \quad Q_{\mu}^{\text {lat }} \psi=-i \partial_{+\mu} \sigma, \quad \tilde{Q}^{\text {lat }} \psi=-\tilde{\sigma}, \\
& Q^{\text {lat }} \tilde{\psi}=\tilde{\sigma}, \quad Q_{\mu}^{\text {lat }} \tilde{\psi}=i \epsilon_{\mu \nu} \partial_{-\nu} \sigma, \quad \tilde{Q}^{\text {lat }} \tilde{\psi}=0, \\
& Q^{\text {lat }} \tilde{\sigma}=0, \quad Q_{\mu}^{\text {lat }} \tilde{\sigma}=i \epsilon_{\mu \nu} \partial_{-\nu} \psi \quad \tilde{Q}^{\text {lat }} \tilde{\phi}=0 . \\
& +i \partial_{+\mu} \tilde{\psi}
\end{aligned}
$$

\section{References}

[1] H. B. Nielsen and M. Ninomiya, "Absence of Neutrinos on a Lattice I. Proof by Homotopy Theory" Nucl. Phys. B185 (1981) 20; erratum: B195 (1981) 541; "Absence of Neutrinos on a Lattice II. Intuitive Topology Proof", Nucl. Phys. B193 (1981) 173.

[2] L. H. Karsten and J. Smit, "Lattice Fermions: Species Doubling, Chiral Invariance and the Triangle Anomaly", Nucl. Phys. B183 (1981) 2649.

[3] P. H. Ginsparg and K. G. Wilson, "A Remnant of Chiral Symmetry on the Lattice", Phys. Rev. D25 (1982) 2649. 
[4] D. B. Kaplan, "A Method for Simulating Chiral Fermions on the Lattice", Phys. Lett. B288 (1992) 342.

[5] P. Hasenfratz, "Prospects for perfect actions", Nucl. Phys.(Proc. Suppl.) 63A-C (1998) 53.

H. Neuberger, "More about exactly massless quarks on the lattice", Phys. Lett. B427 (1998) 353.

M. Lüscher, "Exact chiral symmetry on the lattice and the Ginsparg-Wilson relation, Phys. Lett. B428 (1998)342.

[6] A. D'Adda, I. Kanamori, N. Kawamoto and K. Nagata, "Twisted superspace on a lattice," Nucl. Phys. B 707 (2005) 100 [arXiv:hep-lat/0406029]; "Twisted N=2 exact SUSY on the lattice for BF and Wess-Zumino," Nucl. Phys. Proc. Suppl. 140 (2005) 754 [arXiv:heplat/0409092]; "N=D=2 twisted supersymmetry on a lattice," Nucl. Phys. Proc. Suppl. 140 (2005) 757.

[7] A. D'Adda, I. Kanamori, N. Kawamoto and K. Nagata, "Exact extended supersymmetry on a lattice: Twisted $\mathrm{N}=2$ super Yang-Mills in two dimensions," Phys. Lett. B 633 (2006) 645 [arXiv:hep-lat/0507029].

[8] A. D'Adda, I. Kanamori, N. Kawamoto and K. Nagata, "Exact Extended Supersymmetry on a Lattice: Twisted N=4 Super Yang-Mills in Three Dimensions," Nucl. Phys. B 798, 168 (2008) [arXiv:0707.3533 [hep-lat]]; "Lattice Formulation of the N=4 D=3 Twisted Super Yang-Mills," PoS LAT2007 (2007) 271 [arXiv:0709.0722 [hep-lat]].

[9] S. Catterall, "A Geometrical approach to N=2 super Yang-Mills theory on the two dimensional lattice.", JHEP0411:006 (2004); "Dirac-Kähler fermions and exact lattice supersymmetry", PoSLAT2005 (2006) 006 [arXiv:0509136[hep-lat]].

[10] N. Kawamoto and T. Tsukioka, "N = 2 supersymmetric model with Dirac-Kähler fermions from generalized gauge theory in two dimensions," Phys. Rev. D 61 (2000) 105009 [arXiv:hep-th/9905222].

J. Kato, N. Kawamoto and Y. Uchida, "Twisted superspace for $\mathrm{N}=\mathrm{D}=2$ super BF and Yang-Mills with Dirac-Kähler fermion mechanism," Int. J. Mod. Phys. A 19 (2004) 2149 [arXiv:hep-th/0310242].

J. Kato, N. Kawamoto and A. Miyake, "N = 4 twisted superspace from Dirac-Kähler twist and off-shell SUSY invariant actions in four dimensions," Nucl. Phys. B 721 (2005) 229 [arXiv:hep-th/0502119].

J. Kato and A. Miyake, "Topological hypermultiplet on $\mathrm{N}=2$ twisted superspace in four dimensions," Mod. Phys. Lett. A 21 (2006) 2569 [arXiv:hep-th/0512269]; "Vafa-Witten Theory on N=2 and N=4 Twisted Superspace in Four Dimensions," arXiv:0808.2538 [hepth].

J. Saito, "Superspace Formulation of $N=4$ Super Yang-Mills Theory with a Central Charge," Soryushiron Kenkyu (Kyoto) 111 (2005) 117, [arXive:hep-th/0512226].

[11] N. Marcus, "The Other topological twisting of N=4 Yang-Mills", Nucl. Phys. B452 (1995) 331, [arXiv:hep-th/9506002].

[12] K. Nagata, "On the Continuum and Lattice Formulations of N=4 D=3 Twisted Super Yang-Mills," JHEP 0801 (2008) 041 [arXiv:0710.5689 [hep-th]].

K. Nagata and Y. S. Wu, "Twisted SUSY Invariant Formulation of Chern-Simons Gauge Theory on a Lattice," Phys. Rev. D 78 (2008) 065002 [arXiv:0803.4339 [hep-lat]]. 
[13] K. Nagata, "Exact Lattice Supersymmetry at Large N," JHEP 0810 (2008) 036 [arXiv:0805.4235 [hep-lat]].

[14] S. Elitzur, E. Rabinovici and A. Schwimmer, "Supersymmetric Models On The Lattice" Phys. Lett. B 199 (1982) 165.

N. Sakai and M. Sakamoto, "Lattice Supersymmetry And The Nicolai Mapping" Nucl. Phys. B229 (1983) 173.

V. A. Kostelecky, J. M. Rabin, "Supersymmetry on a superlattice", J. Math. Phys. 25 (1984) 2744.

D. M. Scott, "Lattices, supersymmetry, and Kähler fermions", J. Phys. A17 (1984) 1123.

H. Aratyn, A. H. Zimerman, "Lattice supersymmetry for N=4 Yang-Mills model", J. Phys. A18 (1985)L487.

[15] P. Becher and H. Joos, "The Dirac-Kähler equation and fermions on the lattice", Zeit. Phys. C15 (1982) 343.

T. Banks and P. Windy, "Supersymmetric lattice theories", Nucl. Phys. B198 (1982) 226, J. M. Rabin, "Homology theory of lattice fermion doubling", Nucl. Phys. B201 (1982) 315.

[16] I. Kanamori and N. Kawamoto, "Dirac-Kähler fermion from Clifford product with noncommutative differential form on a lattice," Int. J. Mod. Phys. A 19 (2004) 695 [arXiv:hepth/0305094]; "Dirac-Kähler fermion with noncommutative differential forms on a lattice," Nucl. Phys. Proc. Suppl. 129 (2004) 877 [arXiv:hep-lat/0309120].

[17] W. Pauli, "The Connection Between Spin and Statistics", Phys. Rev. 58 (1940) 716.

[18] P. H. Dondi and H. Nicolai, "Lattice Supersymmetry," Nuovo Cim. A 41 (1977) 1.

[19] I. Montvay, "Supersymmetric Yang-Mills theory on the lattice", Int. J. Mod. Phys. A17 (20002) 2377.

[20] A. Feo, "Supersymmetry on the lattice," Nucl. Phys. Proc. Suppl. 119 (2003) 198 [hep-lat/0112052]; "Supersymmetry on the lattice: Where do predictions and results stand?" [hep-lat/0311037], and references therein; "Predictions and recent results in SUSY on the lattice," Mod. Phys. Lett. A 19 (2004) 2387 [arXiv:hep-lat/0410012].

[21] K. Fujikawa and M. Ishibashi, "Lattice chiral symmetry and the Wess-Zumino model," Nucl. Phys. B622 (2002) 115 [hep-th/0109156], Phys. Lett. B528 (2002) 295 [hep-lat/0112050].

Y. Kikukawa and Y. Nakayama, "Nicolai mapping vs. exact chiral symmetry on the lattice," Phys. Rev. D66 (2002) 094508 [hep-lat/0207013]. K. Fujikawa, "N = 2 Wess-Zumino model on the d = 2 Euclidean lattice," Phys. Rev. D66 (2002) 074510 [hep-lat/0208015]. M. Bonini and A. Feo, "Wess-Zumino model with exact supersymmetry on the lattice," JHEP 0409 (2004) 011 [hep-lat/0402034].

J. W. Elliott and G. D. Moore, "Three dimensional N = 2 supersymmetry on the lattice," JHEP 0511, 010 (2005) [hep-lat/0509032].

[22] K. Itoh, M. Kato, H. Sawanaka, H. So and N. Ukita, "Novel approach to super Yang-Mills theory on lattice: Exact fermionic symmetry and 'Ichimatsu' pattern," JHEP 0302 (2003) 033 [hep-lat/0210049], "Towards the super Yang-Mills theory on the lattice," Prog. Theor. Phys. 108 (2002) 363 [hep-lat/0112052].

[23] H. Suzuki and Y. Taniguchi, "Two-dimensional $\mathrm{N}=(2,2)$ super Yang-Mills theory on the lattice via dimensional reduction," JHEP 0510, 082 (2005) [hep-lat/0507019].

H. Suzuki, "Two-dimensional $\mathcal{N}=(2,2)$ super Yang-Mills theory on computer," 
arXiv:0706.1392 [hep-lat].

Y. Kikukawa and H. Suzuki, "A local formulation of lattice Wess-Zumino model with exact U(1)R symmetry," JHEP 0502 (2005) 012 [hep-lat/0412042].

M. Harada and S. Pinsky, " $N=1$ super Yang-Mills on a $(3+1)$ dimensional transverse lattice with one exact supersymmetry," Phys. Rev. D 71 (2005) 065013 [hep-lat/0411024].

[24] K. Fujikawa, "Supersymmetry on the lattice and the Leibniz rule," Nucl. Phys. B 636 (2002) 80 [arXiv:hep-th/0205095].

[25] J. Nishimura, "Four-dimensional N = 1 supersymmetric Yang-Mills theory on the lattice without fine-tuning", Phys. Lett. B406 (1997) 215 [hep-lat/9701013].

N. Maru and J. Nishimura, "Lattice formulation of supersymmetric Yang-Mills theories without fine-tuning", Int. J. Mod. Phys. A13 (1998) 2841 [hep-th/9705152].

H. Neuberger, "Vector like gauge theories with almost massless fermions on the lattice" Phys. Rev. D57 (1998) 5417 [hep-lat/9710089].

D. B. Kaplan and M. Schmaltz, "Supersymmetric Yang-Mills theories from domain wall fermions" Chin. J. Phys. 38 (2000) 543 [hep-lat/0002030].

G. T. Fleming, J. B. Kogut and P. M. Vranas, "Super Yang-Mills on the lattice with domain wall fermions" Phys. Rev. D64 (2001) 034510 [hep-lat/0008009].

[26] Y. Igarashi, H. So and N. Ukita, "Ginsparg-Wilson relation and lattice chiral symmetry in fermionic interacting theories", Phys. Lett. B535 (2002) 363 [hep-lat/0203019].

G. Bergner, T. Kaestner, S. Uhlmann, and A. Wipf, "Low-dimensional supersymmetric lattice models", Annals Phys. 323 (2008) 946 [arXiv:0705.2212].

T. Kastner, G. Bergner, S. Uhlmann, A. Wipf, and C. Wozar, "Two-Dimensional Wess-Zumino Models at Intermediate Couplings", Phys. Rev. D78 (2008) 095001 [arXiv:0807.1905].

F. Synatschke, G. Bergner, H. Gies, and A. Wipf, "Flow Equation for Supersymmetric Quantum Mechanics", [arXiv:0809.4396v2].

[27] G. Bergner, F. Bruckmann, and J. M. Pawlowski, "Generalising the Ginsparg-Wilson relation: Lattice supersymmetry from blocking transformations", [arXiv:0807.1110].

[28] D. B. Kaplan, E. Katz and M. Unsal, "Supersymmetry on a spatial lattice," JHEP 0305 (2003) 037 [arXiv:hep-lat/0206019].

D. B. Kaplan, "Lattice supersymmetry," Nucl. Phys. Proc. Suppl. 119 (2003) 900 [arXiv:hep-lat/0208046].

A. G. Cohen, D. B. Kaplan, E. Katz and M. Unsal, "Supersymmetry on a Euclidean spacetime lattice. I: A target theory with four supercharges," JHEP 0308 (2003) 024 [arXiv:heplat/0302017]; "Supersymmetry on a Euclidean spacetime lattice. II: Target theories with eight supercharges," JHEP 0312 (2003) 031 [arXiv:hep-lat/0307012].

D. B. Kaplan, "Recent developments in lattice supersymmetry," Nucl. Phys. Proc. Suppl. 129 (2004) 109 [arXiv:hep-lat/0309099].

D. B. Kaplan and M. Unsal, "A Euclidean lattice construction of supersymmetric YangMills theories with sixteen supercharges," JHEP 0509 (2005) 042 [arXiv:hep-lat/0503039]. M. G. Endres and D. B. Kaplan, "Lattice formulation of $(2,2)$ supersymmetric gauge theories with matter fields," JHEP 0610 (2006) 076 [arXiv:hep-lat/0604012].

[29] M. Unsal, "Compact gauge fields for supersymmetric lattices," JHEP 0511, 013 (2005) [arXiv:hep-lat/0504016]; "Supersymmetric deformations of type IIB matrix model as matrix regularization of $\mathrm{N}=4 \mathrm{SYM}$," JHEP 0604, 002 (2006) [arXiv:hep-th/0510004]; "Twisted supersymmetric gauge theories and orbifold lattices," JHEP 0610, 089 (2006) [arXiv:hepth/0603046]. 
[30] S. Catterall and S. Karamov, "Exact lattice supersymmetry: the two-dimensional N = 2 Wess-Zumino model," Phys. Rev. D65 (2002) 094501 [hep-lat/0108024]; "A lattice study of the two-dimensional Wess Zumino model," Phys. Rev. D68 (2003) 014503 [hep-lat/0305002].

S. Catterall, "Lattice supersymmetry and topological field theory," JHEP 0305 (2003) 038 [hep-lat/0301028].

S. Catterall and S. Ghadab, "Lattice sigma models with exact supersymmetry," JHEP 0405 (2004) 044 [hep-lat/0311042], "Twisted supersymmetric sigma model on the lattice," JHEP 0610, 063 (2006) [hep-lat/0607010].

S. Catterall, "A geometrical approach to $\mathrm{N}=2$ super Yang-Mills theory on the two dimensional lattice," JHEP 0411 (2004) 006 [hep-lat/0410052]; "LATTICE FORMULATION OF N=4 SUPER YANG-MILLS THEORY," JHEP 0506, 027 (2005) [hep-lat/0503036]; "Simulations of $\mathcal{N}=2$ super Yang-Mills theory in two dimensions," JHEP 0603 (2006) 032 [hep-lat/0602004]; JHEP 0704, 015 (2007) [hep-lat/0612008].

S. Catterall and T. Wiseman, "Towards lattice simulation of the gauge theory duals to black holes and hot strings," arXiv:0706.3518 [hep-lat].

[31] F. Sugino, "A lattice formulation of super Yang-Mills theories with exact supersymmetry," JHEP 0401 (2004) 015 [hep-lat/0311021]; "Super Yang-Mills theories on the twodimensional lattice with exact supersymmetry," JHEP 0403 (2004) 067 [hep-lat/0401017]; "Various super Yang-Mills theories with exact supersymmetry on the lattice," JHEP 0501 (2005) 016 [hep-lat/0410035]; "Two-Dimensional Compact N=(2,2) Lattice Super YangMills Theory with Exact Supersymmetry," Phys.Lett. B635 (2006) 218 [hep-lat/0601024].

[32] T. Onogi and T. Takimi, Phys. Rev. D 72 (2005) 074504 [arXiv:hep-lat/0506014].

K. Ohta and T. Takimi, "Lattice formulation of two dimensional topological field theory," Prog. Theor. Phys. 117, 317 (2007) [hep-lat/0611011].

T. Takimi, "Relationship between various supersymmetric lattice models," arXiv:0705.3831 [hep-lat].

[33] J. Giedt, "Advances and applications of lattice supersymmetry", PoSLAT2006 008,2006 [hep-lat/0701006]; "R-symmetry in the q-exact (2,2) 2d lattice Wess-Zumino model", Nucl. Phys. B726 (20005) 210 [hep-lat/0507016]; "Deconstruction and other approaches to supersymmetric lattice field theories". Int. J. Mod. Phys. A21 (2006) 3039 [hep-lat/0602007].

[34] P. H. Damgaard and S. Matsuura, "Classification of Supersymmetric Lattice Gauge Theories by Orbifolding," JHEP 0707 (2007) 051 [arXiv:0704.2696 [hep-lat]]; "Relations among Supersymmetric Lattice Gauge Theories via Orbifolding," JHEP 0708 (2007) 087 [arXiv:0706.3007 [hep-lat]]; "Lattice Supersymmetry: Equivalence between the Link Approach and Orbifolding," JHEP 0709 (2007) 097 [arXiv:0708.4129 [hep-lat]]; "Geometry of Orbifolded Supersymmetric Lattice Gauge Theories," Phys. Lett. B 661 (2008) 52 [arXiv:0801.2936 [hep-th]].

[35] M. Unsal, "Deformed matrix models, supersymmetric lattice twists and $N=1 / 4$ supersymmetry," arXiv:0809.3216 [hep-lat].

[36] S. Catterall, D. Kaplan, and M. Unsal, "Exact lattice supersymmetry", [arXiv:0903.4881v1].

[37] D. B. Kaplan, "Dynamical Generation Of Supersymmetry" Phys. Lett. B136 (1984) 162. 
[38] H. Fukaya, I. Kanamori, H. Suzuki, M. Hayakawa and T. Takimi, "Note on massless bosonic states in two-dimensional field theories," Prog. Theor. Phys. 116, 1117 (2007) [arXiv:hepth/0609049].

H. Fukaya, I. Kanamori, H. Suzuki and T. Takimi, "Numerical results of two-dimensional N=(2,2) super Yang-Mills theory," PoS LAT2007, 264 (2007) [arXiv:0709.4076 [hep-lat]]. I. Kanamori, H. Suzuki and F. Sugino, "Euclidean lattice simulation for the dynamical supersymmetry breaking," Phys. Rev. D 77, 091502 (2008) [arXiv:0711.2099 [hep-lat]]; "Observing dynamical supersymmetry breaking with euclidean lattice simulations," Prog. Theor. Phys. 119, 797 (2008) [arXiv:0711.2132 [hep-lat]].

I. Kanamori, "Observing dynamical SUSY breaking with lattice simulation," AIP Conf. Proc. 1078, 423 (2009) [arXiv:0809.0646 [hep-lat]]; "RHMC simulation of two-dimensional $\mathrm{N}=(2,2)$ super Yang-Mills with exact supersymmetry," arXiv:0809.0655 [hep-lat].

I. Kanamori and H. Suzuki, "Restoration of supersymmetry on the lattice: Twodimensional $\mathcal{N}=(2,2)$ supersymmetric Yang-Mills theory," Nucl. Phys. B 811, 420 (2009) [arXiv:0809.2856 [hep-lat]]; "Some physics of the two-dimensional $\mathcal{N}=(2,2)$ supersymmetric Yang-Mills theory: Lattice Monte Carlo study," Phys. Lett. B 672, 307 (2009) [arXiv:0811.2851 [hep-lat]].

I. Kanamori, "Vacuum energy of two-dimensional $\mathrm{N}=(2,2)$ super Yang-Mills theory," arXiv:0902.2876 [hep-lat].

[39] E. Witten, "Topological Quantum Field Theory," Commun. Math. Phys. 117 (1988) 353; "Topological Sigma Models," Commun. Math. Phys. 118 (1988) 411.

[40] S. Arianos, A. D'Adda, N. Kawamoto and J. Saito, "Lattice supersymmetry in 1D with two supercharges," PoS LATTICE2007 (2006) 259 [arXiv:0710.0487 [hep-lat]].

S. Arianos, A. D'Adda, A. Feo, N. Kawamoto and J. Saito, "Matrix formulation of superspace on 1D lattice with two supercharges," arXiv:0806.0686 [hep-lat].

[41] R. Oeckl, "Braided quantum field theory," Commun. Math. Phys. 217 (2001) 451 [arXiv:hep-th/9906225]; "Introduction To Braided Quantum Field Theory," Int. J. Mod. Phys. B 14 (2000) 2461.

[42] F. Bruckmann and M. de Kok, "Noncommutativity approach to supersymmetry on the lattice: SUSY quantum mechanics and an inconsistency," Phys. Rev. D 73 (2006) 074511 [arXiv:hep-lat/0603003].

F. Bruckmann, S. Catterall and M. de Kok, "A critique of the link approach to exact lattice supersymmetry," Phys. Rev. D 75 (2007) 045016 [arXiv:hep-lat/0611001].

[43] M. Kato, M. Sakamoto and H. So, "Taming the Leibniz Rule on the Lattice," JHEP 0805, 057 (2008) [arXiv:0803.3121 [hep-lat]]; "Leibniz rule and exact supersymmetry on lattice: A case of supersymmetrical quantum mechanics," PoS LAT2005 (2006) 274 [arXiv:heplat/0509149]; "No-Go Theorem of Leibniz Rule and Supersymmetry on the Lattice," arXiv:0810.2360 [hep-lat].

[44] S. D. Drell, M. Weinstein and S. Yankielowicz, "Strong Coupling Field Theories. 2. Fermions And Gauge Fields On A Lattice," Phys. Rev. D 14 (1976) 1627.

[45] K. Osterwalder and R. Schrader, "AXIOMS FOR EUCLIDEAN GREEN'S FUNCTIONS," Commun. Math. Phys. 31 (1973) 83;

[46] K. Osterwalder and R. Schrader, "Axioms For Euclidean Green's Functions. 2," Commun. Math. Phys. 42 (1975) 281. 
[47] K. Osterwalder and E. Seiler, "Gauge Field Theories On The Lattice," Annals Phys. 110 (1978) 440.

[48] N. Kawamoto and J. Smit, "Effective Lagrangian and dynamical symmetry breaking in strongly coupled lattice QCD ", Nucl. Phys. B192 (1981), 100.

[49] F. Gliozzi, "Spinor algebra of the one component lattice fermions", Nucl. Phys. B204 (1982), 419.

[50] H. Kluberg-Stern, A. Morel, O. Napoly, and B. Petersson, "Flavors of Lagrangian Susskind fermions", Nucl. Phys. B220 (1983), 447.

[51] J. B. Kogut and L. Susskind, Phys. Rev. D11 (1975) 395, L. Susskind, Phys. Rev. D16 (1977) 3031.

[52] M. Chaichian, P. P. Kulish, K. Nishijima and A. Tureanu, "On a Lorentz-invariant interpretation of noncommutative space-time and its implications on noncommutative QFT," Phys. Lett. B 604 (2004) 98 [arXiv:hep-th/0408069].

J. Wess, "Deformed coordinate spaces: Derivatives," arXiv:hep-th/0408080.

F. Koch and E. Tsouchnika, "Construction of theta-Poincare algebras and their invariants on M(theta)," Nucl. Phys. B 717 (2005) 387 [arXiv:hep-th/0409012].

$\mathrm{R}$. Oeckl, "Untwisting noncommutative $\mathrm{R}^{* *} \mathrm{~d}$ and the equivalence of quantum field theories," Nucl. Phys. B 581 (2000) 559 [arXiv:hep-th/0003018].

M. Chaichian, P. Presnajder and A. Tureanu, "New concept of relativistic invariance in NC space-time: Twisted Poincare symmetry and its implications," Phys. Rev. Lett. 94 (2005) 151602 [arXiv:hep-th/0409096].

G. Fiore and J. Wess, "On 'full' twisted Poincare' symmetry and QFT on Moyal-Weyl spaces," Phys. Rev. D 75 (2007) 105022 [arXiv:hep-th/0701078].

A. P. Balachandran, A. R. Queiroz, A. M. Marques and P. Teotonio-Sobrinho, "Quantum Fields with Noncommutative Target Spaces," Phys. Rev. D 77 (2008) 105032 [arXiv:0706.0021 [hep-th]].

[53] T. Asakawa, M. Mori and S. Watamura, "Hopf Algebra Symmetry and String," arXiv:0805.2203 [hep-th]; "Twist Quantization of String and B Field Background," arXiv:0811.1638 [hep-th].

[54] Y. Sasai and N. Sasakura, "Braided quantum field theories and their symmetries," Prog. Theor. Phys. 118 (2007) 785 [arXiv:0704.0822 [hep-th]].

[55] M. Riccardi and R. J. Szabo, "Duality and Braiding in Twisted Quantum Field Theory," JHEP 0801 (2008) 016 [arXiv:0711.1525 [hep-th]].

[56] S. Majid, "Examples of braided groups and braided matrices," J. Math. Phys. 32 (1991) 3246; "Quantum and braided linear algebra," J. Math. Phys. 34 (1993) 1176 [arXiv:hepth/9208006]; "Braided momentum in the Q Poincare group," J. Math. Phys. 34 (1993) 2045 [arXiv:hep-th/9210141]; "Free braided differential calculus, braided binomial theorem and the braided exponential map," J. Math. Phys. 34 (1993) 4843 [arXiv:hep-th/9302076]; "Beyond supersymmetry and quantum symmetry: An Introduction to braided groups and braided matrices," arXiv:hep-th/9212151;

A. Kempf and S. Majid, "Algebraic q integration and Fourier theory on quantum and braided spaces," J. Math. Phys. 35 (1994) 6802 [arXiv:hep-th/9402037].

[57] I. Bars and D. Minic, "Non-commutative geometry on a discrete periodic lattice and gauge theory," Phys. Rev. D 62, 105018 (2000) [arXiv:hep-th/9910091]. 
[58] J. Ambjorn, Y. M. Makeenko, J. Nishimura and R. J. Szabo, "Finite N matrix models of noncommutative gauge theory," JHEP 9911 (1999) 029 [arXiv:hep-th/9911041]; "Nonperturbative dynamics of noncommutative gauge theory," Phys. Lett. B 480 (2000) 399 [arXiv:hep-th/0002158]; "Lattice gauge fields and discrete noncommutative Yang-Mills theory," JHEP 0005 (2000) 023 [arXiv:hep-th/0004147].

[59] M. Hanada, J. Nishimura and S. Takeuchi "Non-lattice simulation for supersymmetric gauge theories in one dimension," arXiv:0706.1647 [hep-lat].

J. W. Elliott, J. Giedt and G. D. Moore, Phys. Rev. D 78 (2008) 081701 [arXiv:0806.0013 [hep-lat]].

[60] G. Ishiki, Y. Takayama and A. Tsuchiya, "N $=4 \mathrm{SYM}$ on $\mathrm{R} \times \mathrm{S}^{* *} 3$ and theories with 16 supercharges," JHEP 0610 (2006) 007 [arXiv:hep-th/0605163].

G. Ishiki, S. Shimasaki, Y. Takayama and A. Tsuchiya, "Embedding of theories with $\mathrm{SU}(2$-4) symmetry into the plane wave matrix model," JHEP 0611 (2006) 089 [arXiv:hepth/0610038].

T. Ishii, G. Ishiki, K. Ohta, S. Shimasaki and A. Tsuchiya, "On relationships among Chern-Simons theory, BF theory and matrix model," Prog. Theor. Phys. 119 (2008) 863 [arXiv:0711.4235 [hep-th]].

T. Ishii, G. Ishiki, S. Shimasaki and A. Tsuchiya, "Fiber Bundles and Matrix Models," Phys. Rev. D 77 (2008) 126015 [arXiv:0802.2782 [hep-th]]; "N=4 Super Yang-Mills from the Plane Wave Matrix Model," Phys. Rev. D 78 (2008) 106001 [arXiv:0807.2352 [hep-th]]. G. Ishiki, S. W. Kim, J. Nishimura and A. Tsuchiya, "Deconfinement phase transition in $\mathrm{N}=4$ super Yang-Mills theory on $\mathrm{RxS}^{\wedge} 3$ from supersymmetric matrix quantum mechanics," arXiv:0810.2884 [hep-th].

G. Ishiki, K. Ohta, S. Shimasaki and A. Tsuchiya, "Two-Dimensional Gauge Theory and Matrix Model," arXiv:0811.3569 [hep-th].

[61] M. Chaichian and A. P. Demichev, "Introduction To Quantum Groups," Singapore, Singapore: World Scientific (1996) 343 p.

[62] S. Majid, "Foundations of quantum group theory," Cambridge, UK: Univ. Pr. (1995) 607 p.

[63] V. Chari and A. Pressley, Cambridge, UK: Univ. Pr. (1994) 651 p. 